\title{
$\mathbb{N}<\mathrm{IT}$
}

Karlsruher Institut für Technologie

\section{Regionalität aus Sicht von Energieversorgungsunternehmen - Eine qualitative Inhaltsanalyse zu Regionalstrom in Deutschland}

Nico Lehmann, Jonathan Müller, Armin Ardone, Katharina Karner, Wolf Fichtner

No. 48 | November 2020

\section{WORKING PAPER SERIES IN PRODUCTION AND ENERGY}

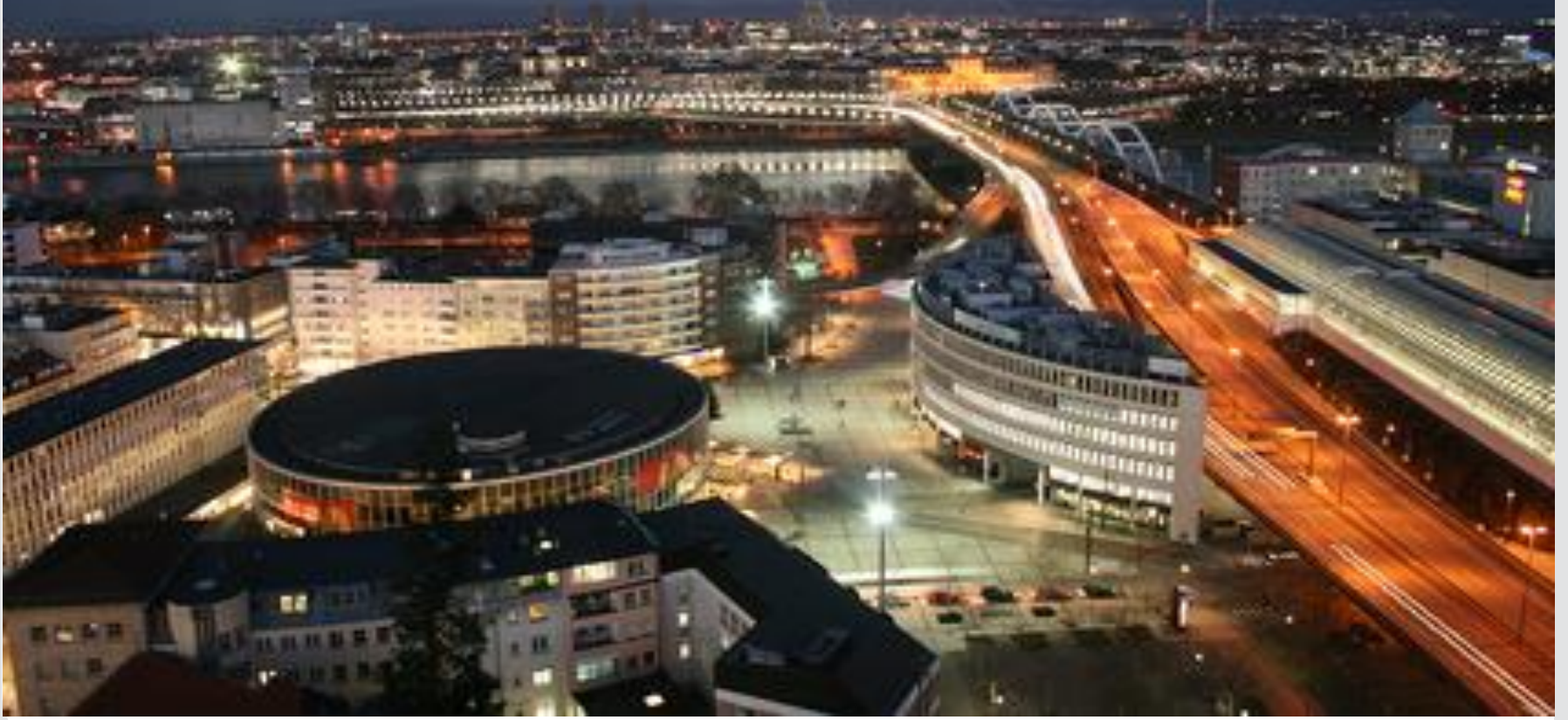




\title{
Regionalität aus Sicht von Energieversorgungsunternehmen - Eine qualitative Inhaltsanalyse zu Regionalstrom in Deutschland
}

\author{
Nico Lehmann, Jonathan Müller, Armin Ardone, \\ Katharina Karner, Wolf Fichtner
}

\begin{abstract}
Lehrstuhl für Energiewirtschaft, Institut für Industriebetriebslehre und Industrielle Produktion (IIP), Karlsruher Institut für Technologie (KIT) Geb. 06.33, Hertzstr. 16, 76187 Karlsruhe

Tel.: 0721-608-44573, E-Mail: nico.lehmann@kit.edu
\end{abstract}

\section{Kurzfassung}

Anfang 2019 wurde beim Umweltbundesamt das Regionalnachweisregister ins Leben gerufen, mit dessen Hilfe Energieversorger die Regionalität einer Stromlieferung gegenüber ihren Kunden ausweisen können. Doch auch ohne Regionalnachweisregister wird teilweise mit der Regionalität von Stromtarifen geworben. Dies wirft die Frage auf, wie Regionalstrom aus Sicht von Energieversorgern ökonomisch zu bewerten ist. Im Rahmen dieser Arbeit wurden Experteninterviews mit 17 Vertretern von Energieversorgungsunter-nehmen durchgeführt. Es zeigt sich, dass viele Energieversorger dem Trend folgen, Regionalstrom anzu-bieten. Insbesondere sind laut Experten das Unternehmensimage kommunaler Energieversorger und die Produktaussagen von Regionalstrom stimmig. Dennoch sehen fast alle Interviewpartner Regionalstrom in seiner heutigen Form als Nischenprodukt, das es mit hoher Wahrscheinlichkeit auch bleiben wird. Die aktuelle Regulatorik, im Rahmen derer Regionalstrom als Premiumprodukt mit einem Aufpreis ggü. nicht regionalem Strom vertrieben wird, macht das Produkt für viele Kunden unattraktiv.
Abstract
At the beginning of 2019, the German Environment Agency established the System for Guarantees of Regional Origin (GRO). With the help of the GROs, energy providers can show that a certain proportion or a certain amount of electricity originates from the region. Even without GROs, some energy providers advertise the regionality of their electricity tariffs. This trend raises the question of how to assess regional electricity in economic terms from a provider's perspective. Therefore, we conducted expert interviews with 17 representatives from energy supply companies. The interviews revealed that many energy pro-viders are currently following the trend to offer regional electricity tariffs. According to the experts, especially the corporate image of municipal energy providers seems to be consistent with the product claims of regional electricity. Yet, almost all interviewees see regional electricity in its current form as a niche product, which it will most likely remain if no regulatory changes are made in the future, e.g. dis-counts or subsidies for regional electricity. 


\title{
Regionalität aus Sicht von Energiever- sorgungsunternehmen
}

\section{Eine qualitative Inhaltsanalyse zu Regionalstrom in Deutschland}

\author{
Working Paper \\ von \\ Nico Lehmann, Jonathan Müller, Armin Ardone, \\ Katharina Karner, Wolf Fichtner \\ Institut für Industriebetriebslehre und Industrielle Produktion (IIP) \\ Lehrstuhl für Energiewirtschaft \\ Karlsruher Institut für Technologie (KIT) \\ Geb. 06.33, Hertzstr. 16, 76187 Karlsruhe, Deutschland \\ Tel.: 0721 608-44573, E-Mail: nico.lehmann@kit.edu
}




\section{Kurzfassung}

Anfang 2019 wurde beim Umweltbundesamt das Regionalnachweisregister ins Leben gerufen, mit dessen Hilfe Energieversorger die Regionalität einer Stromlieferung gegenüber ihren Kunden ${ }^{1}$ ausweisen können. Doch auch ohne Regionalnachweisregister wird teilweise mit der Regionalität von Stromtarifen geworben. Dies wirft die Frage auf, wie Regionalstrom aus Sicht von Energieversorgern ökonomisch zu bewerten ist. Im Rahmen dieser Arbeit wurden Experteninterviews mit 17 Vertretern von Energieversorgungsunternehmen durchgeführt. Es zeigt sich, dass viele Energieversorger dem Trend folgen, Regionalstrom anzubieten. Insbesondere sind laut Experten das Unternehmensimage kommunaler Energieversorger und die Produktaussagen von Regionalstrom stimmig. Dennoch sehen fast alle Interviewpartner Regionalstrom in seiner heutigen Form als Nischenprodukt, das es mit hoher Wahrscheinlichkeit auch bleiben wird. Die aktuelle Regulatorik, im Rahmen derer Regionalstrom als Premiumprodukt mit einem Aufpreis ggü. nicht regionalem Strom vertrieben wird, macht das Produkt für viele Kunden unattraktiv.

\footnotetext{
${ }^{1}$ Aus Gründen der leichteren Lesbarkeit wird in der vorliegenden Arbeit die gewohnte männliche Sprachform bei personenbezogenen Substantiven und Pronomen verwendet. Dies impliziert jedoch keine Benachteiligung eines Geschlechts, sondern soll im Sinne der sprachlichen Vereinfachung als geschlechtsneutral zu verstehen sein.
} 


\section{Abstract}

At the beginning of 2019, the German Environment Agency established the System for Guarantees of Regional Origin (GRO). With the help of the GROs, energy providers can show that a certain proportion or a certain amount of electricity originates from the region. Even without GROs, some energy providers advertise the regionality of their electricity tariffs. This trend raises the question of how to assess regional electricity in economic terms from a provider's perspective. Therefore, we conducted expert interviews with 17 representatives from energy supply companies. The interviews revealed that many energy providers are currently following the trend to offer regional electricity tariffs. According to the experts, especially the corporate image of municipal energy providers seems to be consistent with the product claims of regional electricity. Yet, almost all interviewees see regional electricity in its current form as a niche product, which it will most likely remain if no regulatory changes are made in the future, e.g. discounts or subsidies for regional electricity. 


\section{Inhaltsverzeichnis}

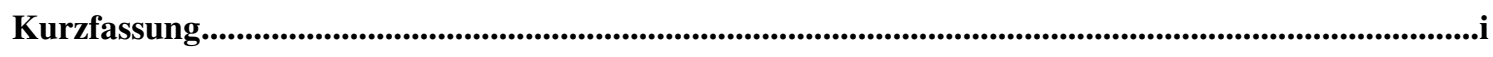

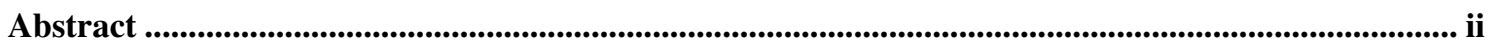

Inhaltsverzeichnis ............................................................................................................................................ iii

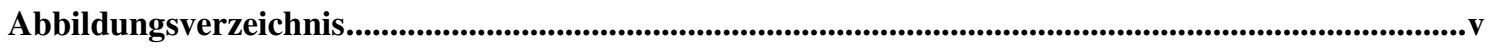

Tabellenverzeichnis ....................................................................................................................................................vi

Abkürzungsverzeichnis .................................................................................................................................... vii

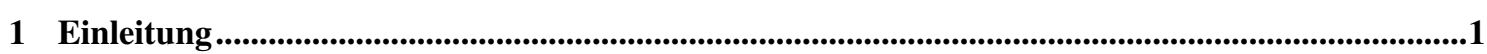

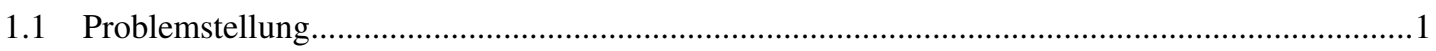

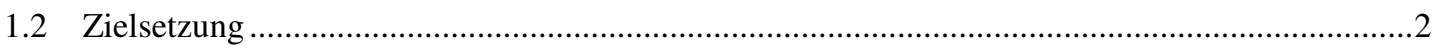

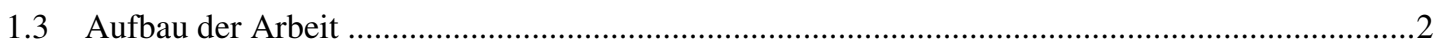

2 Grundlagen ................................................................................................................................

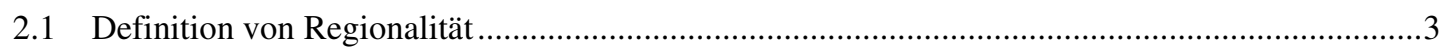

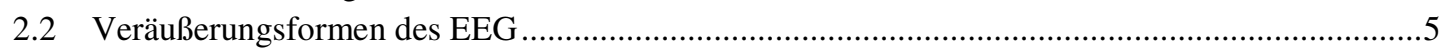

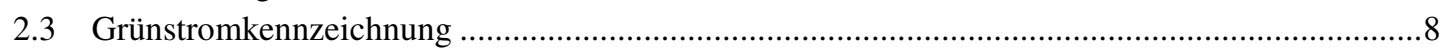

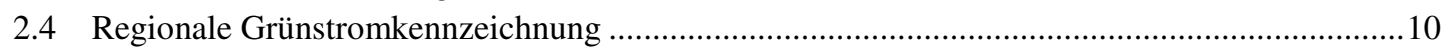

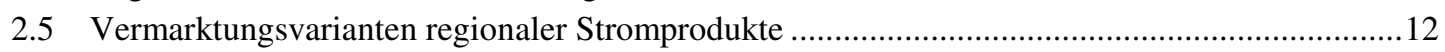

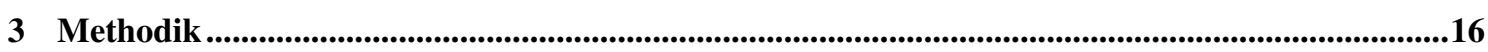

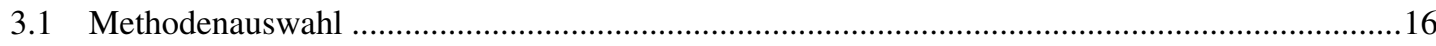

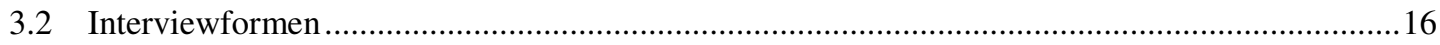

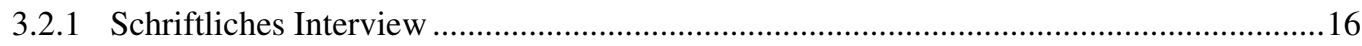

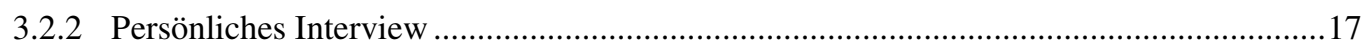

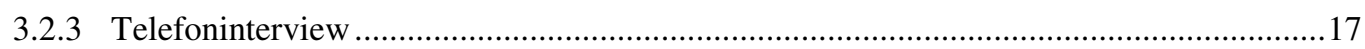

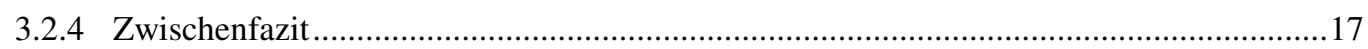

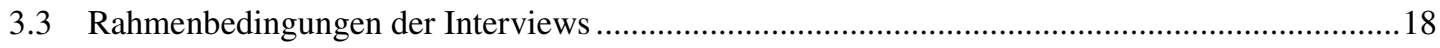

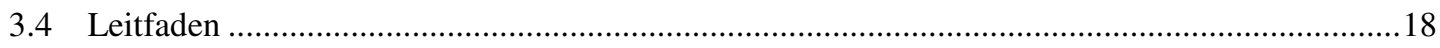

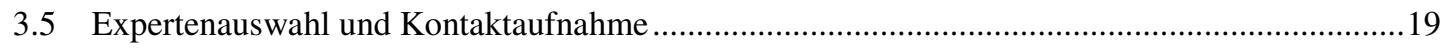

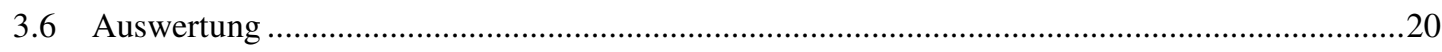

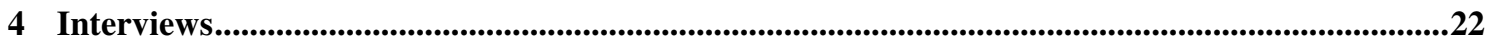

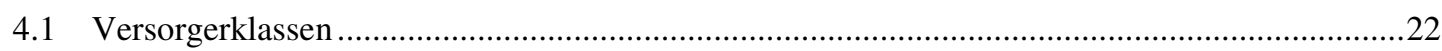

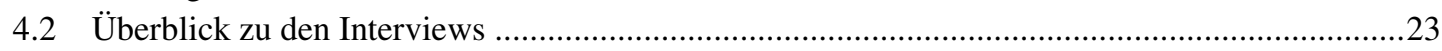

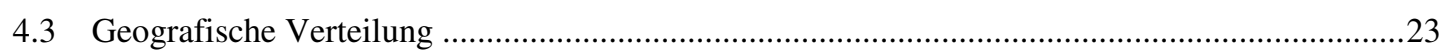

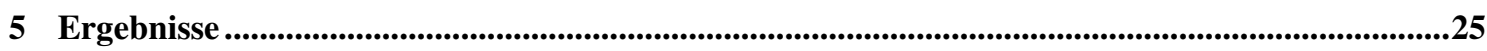

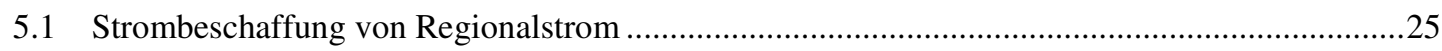

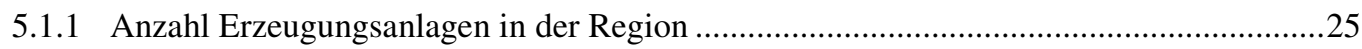

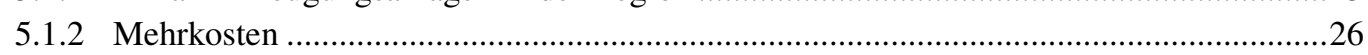

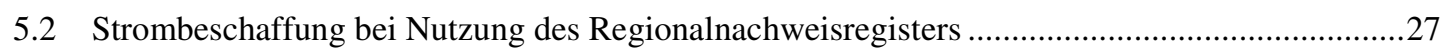

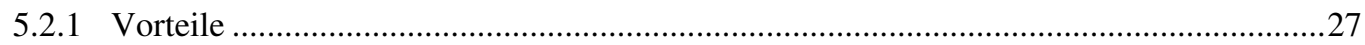

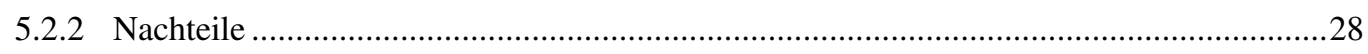

5.2.3 Komplexität für Energieversorgungsunternehmen ....................................................... 
5.2.4 Kopplung der Regionalnachweise an die vertragliche Lieferkette...............................31

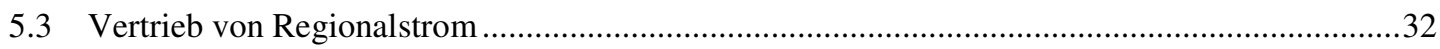

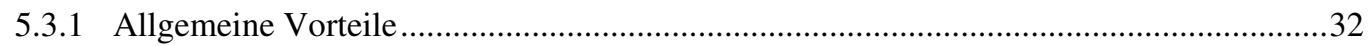

5.3.2 Akzeptanz für Anlagen und Image des Versorgers ......................................................33

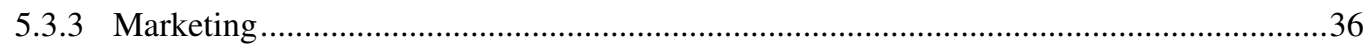

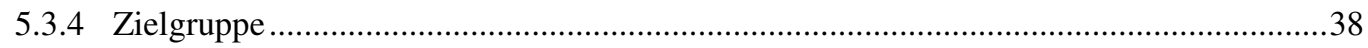

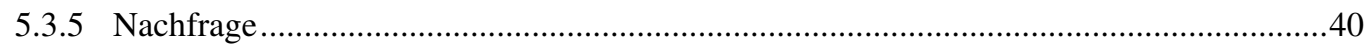

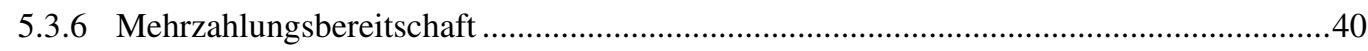

5.4 Vertrieb bei Nutzung des Regionalnachweisregisters .......................................................42

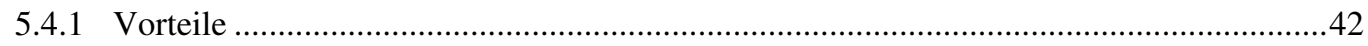

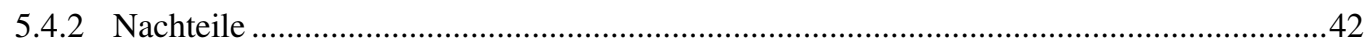

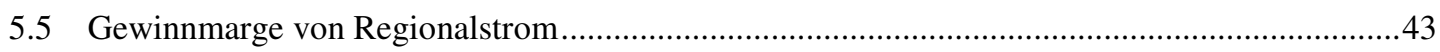

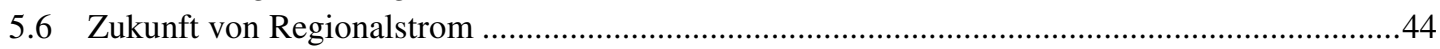

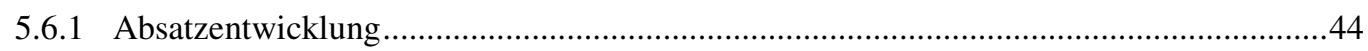

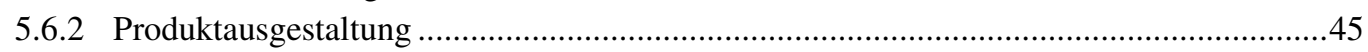

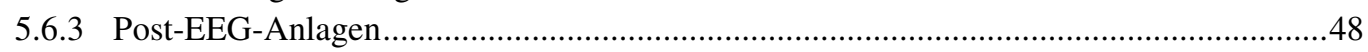

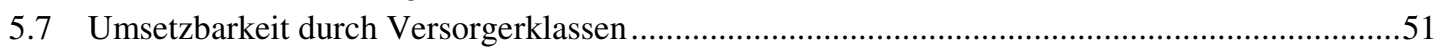

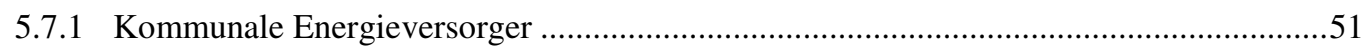

5.7.2 Bürgernahe Energieversorger .................................................................................52

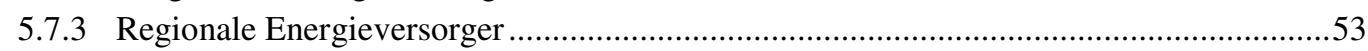

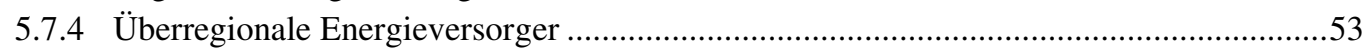

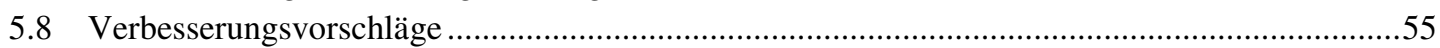

5.8.1 Regionalstrom bei Nutzung des Regionalnachweisregisters..........................................55

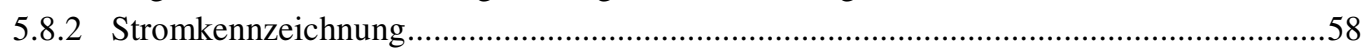

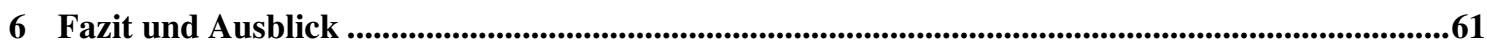

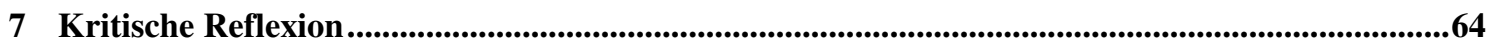

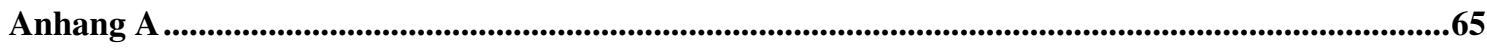

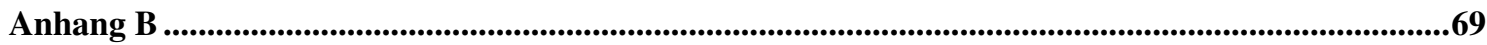

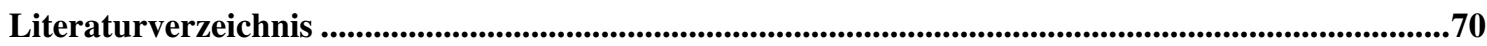




\section{Abbildungsverzeichnis}

Abbildung 1: $\quad$ Definition der Region des RNR bei Gemeinden mit mehreren Postleitzahlengebieten....4

Abbildung 2: $\quad$ Eingespeiste Arbeit im Jahr 2018 in der Veräußerungsform der Einspeisevergütung ......6

Abbildung 3: $\quad$ Eingespeiste Arbeit im Jahr 2018 in der Veräußerungsform der Marktprämie ...............7

Abbildung 4: $\quad$ Eingespeiste Arbeit im Jahr 2018 in der Veräußerungsform der sonstigen

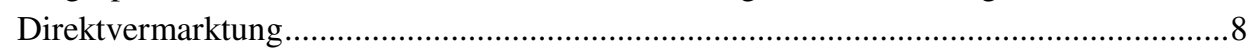

Abbildung 5: Vermarktungsvarianten regionaler Stromprodukte $\quad$.................................................13

Abbildung 6: Exemplarische Stromkennzeichnung ohne und mit Nutzung des Regionalnachweisregisters .............................................................................. 14

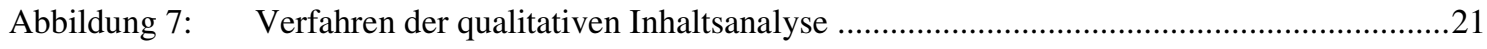

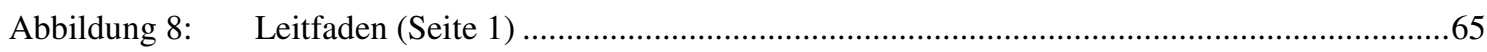

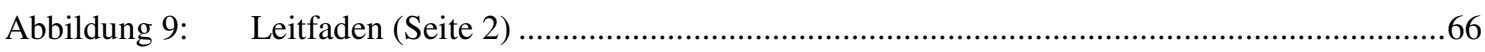

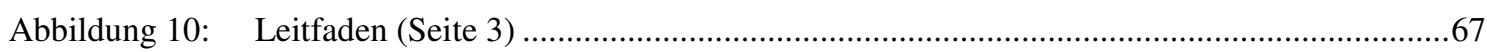

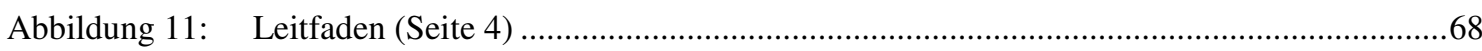

Abbildung 12: Anschreiben an (potenzielle) Interviewpartner ..........................................................69 


\section{Tabellenverzeichnis}

Tabelle 1: Überblick zu den durchgeführten Interviews...

Tabelle 2: Geografische Verteilung der Interviewpartner 


\section{Abkürzungsverzeichnis}

B

BAFA Bundesamt für Wirtschaft und Ausfuhrkontrolle

BDEW Bundesverband der Energie- und

Wasserwirtschaft e. V.

BMWi Bundesministerium für Wirtschaft und Energie

\section{$\mathbf{E}$}

EEG Erneuerbaren-Energien-Gesetz

EnWG Energiewirtschaftsgesetz

EU Europäische Union

EuGH Europäischer Gerichtshof

EVU Energieversorgungsunternehmen

\section{H}

HKN Herkunftsnachweis(e)

HKNR Herkunftsnachweisregister

HkRNDV Durchführungsverordnung über

Herkunfts- und Regionalnachweise für Strom aus erneuerbaren Energien

$\mathbf{K}$

KI Künstliche Intelligenz

kW Kilowatt

kWh Kilowattstunde

KWK Kraft-Wärme-Kopplung
KWK-G Kraft-Wärme-Kopplungsgesetz

$\mathbf{L}$

LV Letztverbraucher

M

MWh Megawattstunde

$\mathbf{0}$

OTC Over-The-Counter

\section{$\mathbf{P}$}

PPA Power Purchase Agreement

$\mathbf{R}$

RN Regionalnachweis(e)

RNR Regionalnachweisregister

$\mathbf{S}$

StromStG Stromsteuergesetz

$\mathbf{U}$

UBA Umweltbundesamt

UWG Gesetz gegen den unlauteren Wettbewerb 


\section{Einleitung}

Dieses Kapitel legt die Problemstellung, die Zielsetzung sowie den Aufbau der Arbeit dar.

\subsection{Problemstellung}

Das Regionalnachweisregister (RNR) wurde am 01.01.2019 in Deutschland eingeführt. Das RNR ermöglicht es Betreibern von Erneuerbaren-Energien-Anlagen Regionalnachweise (RN) für Strom aus ihren Anlagen ausstellen zu lassen, sofern sich die Anlagen in der Veräußerungsform der Marktprämie (§ 19 Abs. 1 Nr. 2 Erneuerbare-Energien-Gesetz (EEG) befinden. Zwar wurden Stromprodukte ${ }^{1}$ bzw. Stromtarife ${ }^{2}$ mit der Eigenschaft Regionalität auch schon vor der Einführung des RNR von Energieversorgungsunternehmen (EVU) angeboten, doch konnte diese Eigenschaft nicht in der Stromkennzeichnung für Letztverbraucher (LV) ausgewiesen werden. Weiterhin mangelte es an einer einheitlichen Definition, was unter Regionalität im Kontext von Stromtarifen zu verstehen ist. Durch das RNR kann nun die Regionalität im Rahmen der Stromkennzeichnung ggü. dem Letztverbraucher ausgewiesen werden. Für diesen ist damit in der Jahresabschlussrechnung nicht nur ersichtlich, aus welchen Energiequellen der bezogene Strom stammt, sondern auch, in welcher Höhe Strom aus der Region geliefert wurde. Ferner existiert durch das RNR nun eine einheitliche Regionalitätsdefinition. Regionalität ist definiert als Umkreis von 50 Kilometern um das Postleitzahlengebiet des Letztverbrauchers ${ }^{3}$ (§ 79a Abs. 6 EEG). Aufgrund der Beschränkung des RNR auf Anlagen in der Marktprämie können Anlagen in der Veräußerungsform der Einspeisevergütung (§21 Abs. 1, 2 EEG) und der sonstigen Direktvermarktung ( $\$ 21$ a EEG) keine RN ausstellen. Letzteres sind Anlagen, die nie unter die Förderung des EEG gefallen sind ${ }^{4}$, deren Förderzeitraum von 20 Jahren ausgelaufen ist ${ }^{5}$ oder die freiwillig auf die EEG-Förderung verzichten. Zwar produzieren diese ggf. auch regional Strom, doch dürfen diese Anlagen aufgrund fehlender Nachweise diesen nicht in der Stromkennzeichnung ausweisen [4]. Die verschiedenen Möglichkeiten, Stromprodukte mit der Eigenschaft Regionalität zu versehen (siehe Kapitel 2.2), erschwert das Verständnis auf der Verbraucherseite, was unter Regionalstrom zu verstehen ist. Diese Forschungsarbeit führt eine Betrachtung des Themas Regionalstrom aus Sicht von Energieversorgungsunternehmen durch, um einen Überblick zu geben, wie sich die Branche in Deutschland zu diesem Thema positioniert. Dabei stehen der Vertrieb und der Stromeinkauf im Fokus und damit zwei Organisationseinheiten, die sowohl Einblicke in das Endkundengeschäft als auch die Beschaffungsseite ermöglichen.

\footnotetext{
${ }^{1}$ In Anlehnung an [1, S. 83] werden Stromprodukte definiert als Stromlieferungen, die Endkunden von EVU in Form eines oder mehrerer Stromtarife angeboten werden. Stromprodukte unterscheiden sich im Portfolio eines EVU hinsichtlich Eigenschaften, die ebenfalls Bestandteil eines Stromtarifs sein können, z.B. dem Strommix oder der Stromherkunft.

${ }^{2}$ Stromtarife sind Stromprodukte, die Endkunden eines EVU angeboten werden [1, S. 83]. Es können, müssen jedoch nicht alle Merkmale eines Stromprodukts Teil des Liefervertrags sein. Weiterhin können weitere Eigenschaften hinzukommen, wie bspw. Preise, Preisgarantien oder Mindestvertragslaufzeiten.

${ }^{3}$ Letztverbraucher ist jede natürliche oder juristische Person, die Strom verbraucht ( 3 Nr. 33 EEG). Im Rahmen dieser Arbeit wird die Bezeichnung (End)Kunde synonym verwendet. Jedoch setzt die Bezeichnung (End)Kunde zusätzlich eine Geschäftsbeziehung zwischen Letztverbraucher und EVU voraus [vgl. 2].

${ }^{4}$ Dies sind insbesondere Laufwasser- und Speicherkraftwerke, die bereits vor der Einführung des EEG in Betrieb genommen wurden.

${ }^{5}$ Die ersten Anlagen scheiden 2021 aus der EEG-Förderung aus [3].
} 


\subsection{Zielsetzung}

Diese Forschungsarbeit widmet sich dem Thema Regionalstrom in Deutschland aus der Perspektive von Energieversorgungsunternehmen und hat zum Ziel, folgende Forschungsfrage zu beantworten:

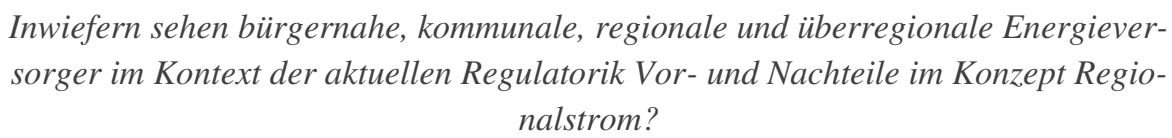

Zur Beantwortung dieser Forschungsfrage wurden 17 Experteninterviews mit leitenden Angestellten des Vertriebs, des Stromeinkaufs, Geschäftsführern sowie Mitarbeitern, die sich mit neuen Geschäftsfeldern auseinandersetzen, verschiedener Energieversorgungsunternehmen in Deutschland durchgeführt. Darunter befinden sich sowohl bürgernahe, kommunale, regionale als auch überregionale Energieversorger (siehe Kapitel 4.1). Auf Basis der Interviews wird die aufgeworfene Forschungsfrage beantwortet und ein Einblick in die Sichtweise von EVU auf das Konzept Regionalstrom ${ }^{6}$ in Deutschland gegeben. Insbesondere wird auf die Vor- und Nachteile, die mit dem Anbieten von Regionalstrom einhergehen, eingegangen. Letztlich wird basierend auf den Interviews eine Einschätzung getroffen, ob Regionalstrom ein (potenzielles) Geschäftsmodell für Versorger ist. Diese Forschungsarbeit betrachtet Regionalstrom sowohl ohne Nutzung des RNR als auch die Änderungen, die dessen Einführung Anfang 2019 mit sich gebracht hat. Zuletzt betrachtet diese Arbeit die potenzielle Zukunft von Regionalstrom aus Sicht der Experten.

\section{$1.3 \quad$ Aufbau der Arbeit}

Im nachfolgenden Kapitel 2 werden die Grundlagen für das weitere Verständnis dieser Arbeit dargelegt. Kapitel 3 geht auf die Methodik der Arbeit ein und stellt die Vorbereitung, die Durchführung und die Auswertung der Interviews vor. Kapitel 4 gibt einen Überblick über die 17 durchgeführten Interviews. Anschließend werden in Kapitel 5 die Ergebnisse zusammengefasst und mit Zitaten der interviewten Experten unterlegt. Das Fazit, die Beantwortung der Forschungsfrage und ein Ausblick sind in Kapitel 6 zu finden. Das letzte Kapitel 7 schließt mit einer kritischen Reflexion.

\footnotetext{
${ }^{6}$ Der Begriff „Konzept Regionalstrom“ schließt regionale Stromprodukte und regionale Stromtarife mit ein, ist jedoch weiter zu fassen. Dabei ist die Region, ebenso wie die Umsetzung (bspw. klassischer Stromliefervertrag im Endkundengeschäft, Kombination eines Stromliefervertrags mit Beteiligungsmöglichkeiten an Erzeugungsanlagen, Kombination eines Stromliefervertrags mit einem Direktvermarktungsvertrag, etc.) nicht näher bezeichnet. Weiterhin wird der Begriff „Regionalstrom“ synonym verwendet.
} 


\section{Grundlagen}

Dieses Kapitel liegt die für das weitere Verständnis notwendigen Grundlagen dar. Dabei wird zunächst auf die Definition von Regionalität, insbesondere im Kontext der Energiewirtschaft, eingegangen. Die nachfolgenden Unterkapitel adressieren die Veräußerungsformen des EEG, die (regionale) Grünstromkennzeichnung sowie die Vermarktungsvarianten regionaler Stromprodukte. Ein besonderer Fokus wird dabei auf die aktuelle Regulatorik gelegt.

\subsection{Definition von Regionalität}

Insbesondere im Lebensmittelbereich ist ein Trend zu Regionalität zu beobachten [5-9]. Bei einer Umfrage in Deutschland aus dem Jahr 2017 gaben 78 Prozent der Befragten an, dass sie auf Lebensmittel aus der Region Wert legten. Im Jahr zuvor lag dieser Wert bei 73 Prozent [10]. In der Bevölkerung gibt es jedoch keine übereinstimmende Auffassung darüber, was unter Regionalität zu verstehen ist [11]. Vielmehr kann sich die Auffassung je nach Ort oder Individuum unterscheiden. Die Region kann im Kontext von Lebensmitteln nicht nur geographisch, sondern auch historisch-kulturell, politisch-administrativ oder hinsichtlich wirtschaftlicher Kriterien definiert werden [12,13]. Heinze et al. [11] unterscheiden zwischen drei Konzepten, wie Unternehmen Regionalität definieren und damit werben können: (i) Benennung der Region, z.B. „Schwarzwald“, (ii) Festlegung eines metrischen Umkreises, z.B. „,50 Kilometer“, oder (iii) Nennung von (unspezifischen) Begriffen, die einen Regionenbezug vermitteln, z.B. ,aus der Region“.

Die Region als energiewirtschaftlicher Raum ist ebenfalls nicht klar definiert [14-16], wobei auch in diesem Kontext eine an geografische, historisch-kulturelle, politisch-administrative oder infrastrukturelle Gegebenheiten (z.B. Regelzonen, Netzgebiete) angelehnte Zuordnung erfolgen kann [17, S. 83]. In Gesetzestexten finden sich Begriffe, die einen geografischen Bezug vermitteln. Im EEG ist im Rahmen des Eigenverbrauchs selbst erzeugten Stroms sowie des Mieterstromzuschlags in den $\S \S 3 \mathrm{Nr} .19,21$ Abs. 3 Nr. 1 EEG der Begriff des „unmittelbaren räumlichen Zusammenhangs“ zu finden. Bei der Stromweitergabe an Dritte ohne Netzdurchleitung wird in $\S 21 \mathrm{~b}$ Abs. 4 Nr. 2 oder der Anlagenzusammenfassung nach $\S 24$ Abs. 1 Nr. 1 der Begriff der ,unmittelbaren räumlichen Nähe“ verwendet. Im Stromsteuergesetz (StromStG) findet sich in $\S 9$ Abs. 1 Nr. 3 lit. a StromStG der Begriff des „räumlichen Zusammenhangs“ sowie hinsichtlich der Regionalnachweise in § 79a Abs. 8 EEG der ,regionale Zusammenhang“. Weiterhin existieren Begriffe, die neben einem geografischen auch einen funktionalen Bezug voraussetzen, z.B. „,äumlich-funktionaler Zusammenhang“ ( $\$ 48$ Abs. 3 Nr. 3 EEG). Eine einheitliche gesetzliche Definition von Regionalität gibt es, mit Ausnahme der Regelungen zum Regionalnachweisregister, nicht [5, 15, S. 23].

In der wissenschaftlichen Literatur sind in Untersuchungen zu Präferenzen hinsichtlich Eigenschaften von Stromtarifen ebenfalls Regionalitätsdefinitionen zu finden. Eine Unterscheidung zwischen dem der (unbestimmten) Region, dem Heimatland (Schweiz), dem benachbarten Ausland und Osteuropa nehmen Burkhalter et al. [18] vor. Kaenzig et al. [19] unterscheiden zwischen Strom aus der (unbestimmten) Region, aus dem Heimatland Deutschland und dem Ausland. Kalkbrenner et al. [20] definieren Regionalität ausgehend vom Letztverbraucher als Umkreis von 20 Kilometern, wobei zusätzlich ein Regionenbezug über verschiedene Klassen von Energieversorgern hergestellt wird. Ein metrisches Maß nutzen ebenfalls Vecchiato und Tempesta [21] mit drei und zehn Kilometern Distanz zur Erzeugungsanlage. In Günther et al. [22] wird Regionalität beispielhaft anhand eines Windparks beschrieben, der sich in der Nähe der Umfrageteilnehmer befindet. Zudem wird, ebenso wie bei Kalkbrenner et al. [20], über die Versorgerklassen 
ein Regionenbezug hergestellt. Zwei jüngere Erhebungen von Hauser et al. [1] sowie Lehmann und Beikirch [23] nutzen die Definition des § 79a Abs. 6 EEG als Radius von 50 Kilometern um das Postleitzahlengebiet des Letztverbrauchers.

Mit dem Regionalnachweisregister führte der Gesetzgeber Anfang 2019 eine Definition ein, die Regionalität als Distanz zwischen Letztverbraucher und Erzeugungsanlage versteht. Die Region ist definiert als Radius von 50 Kilometern um das Postleitzahlengebiet des Letztverbrauchers, wobei in Fällen, in denen die Gemeinde des Letztverbrauchers mehrere Postleitzahlengebiete umfasst, der 50-Kilometer-Radius erst ab der Gemeindegrenze beginnt ( $\$ 79 \mathrm{a}$ Abs. 6 EEG, $\S 2$ Nr. 12 HkRNDV). ${ }^{1}$ Damit möchte der Gesetzgeber sicherstellen, dass bei größeren Gemeinden ebenfalls genügend Anlagen aus dem Umland zur Verfügung stehen, jedoch auch, dass sich die Region für den Letztverbraucher bei einem Umzug innerhalb der Gemeinde nicht ändert [24]. Sofern der 50-Kilometer-Radius den Rand eines Postleitzahlengebiets schneidet, wird dieses Postleitzahlengebiet ebenfalls zur Region hinzugezählt (§ 2 Nr. 13 HkRNDV). Diese Regelung kann dazu führen, dass die Distanz zwischen Letztverbraucher und Erzeugungsanlage über 50 Kilometer beträgt. Die Regionalitätsdefinition des RNR ist in Abbildung 1 dargestellt.

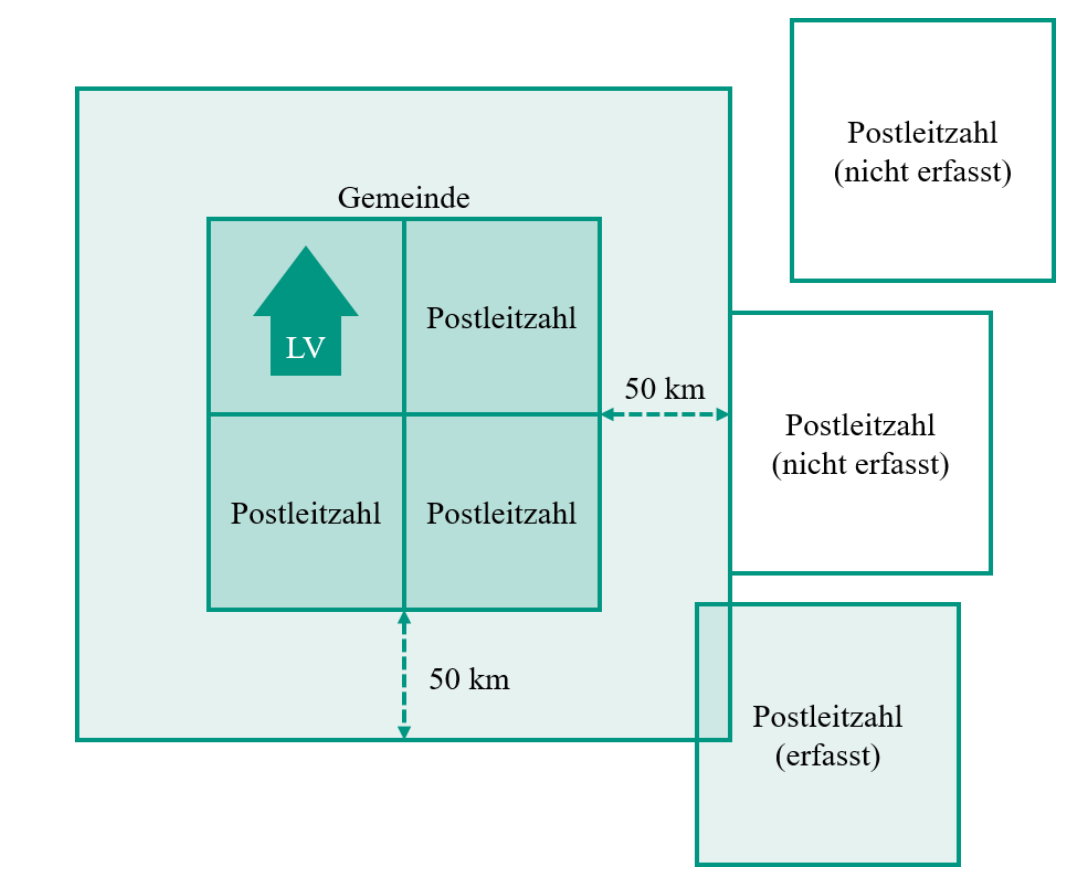

Abbildung 1: Definition der Region des RNR bei Gemeinden mit mehreren Postleitzahlengebieten

Die vom jeweiligen Postleitzahlengebiet ausgehende Region wird auf der Homepage des Umweltbundesamts (UBA) veröffentlicht ( 5 Abs. 1 HkRNDV) und kalenderjährlich aktualisiert ( 5 Abs. 2 HkRNDV). ${ }^{2}$

\footnotetext{
${ }^{1}$ Die Durchführungsverordnung über Herkunfts- und Regionalnachweise für Strom aus erneuerbaren Energien (HkRNDV) nutzt die beiden Begriffe „Verwendungsgebiet“ und „Verwendungsregion“. Das Verwendungsgebiet ist das Postleitzahlengebiet oder das Gemeindegebiet, wenn dieses mehrere Postleitzahlengebiete umfasst, am Ort der Belieferung des Letztverbrauchers mit Strom (§ 2 Nr. 12 HkRNDV). Die Verwendungsregion umfasst das Verwendungsgebiet sowie alle Postleitzahlengebiete, die sich ganz oder teilweise im Umkreis von 50 Kilometern um das Verwendungsgebiet befinden ( 2 Nr. 13 HkRNDV).

${ }^{2}$ Dies wird von Lehnert und Rühr [5] kritisiert, da eine Änderung der Regionen dazu führen kann, dass Erzeugungsanlagen nun nicht mehr für ein Regionalstromprodukt verwendet werden können.
} 
Die vorangegangenen Ausführungen zeigen, dass Regionalität nicht einheitlich definiert ist. Vielmehr muss Regionalität im jeweiligen Kontext verstanden sowie subjektive Vorstellungen berücksichtigt werden. ${ }^{3}$

\subsection{Veräußerungsformen des EEG}

Das EEG ist ein deutsches Gesetz, welches am 01.01.2000 in Kraft trat. Zweck des Gesetzes ist es, im Interesse des Klima- und Umweltschutzes eine nachhaltige Entwicklung der Energieversorgung zu ermöglichen, die volkswirtschaftlichen Kosten der Energieversorgung auch durch die Einbeziehung langfristiger externer Effekte zu verringern, fossile Energieressourcen zu schonen und die Weiterentwicklung von Technologien zur Erzeugung von Strom aus erneuerbaren Energien zu fördern ( 1 Abs. 1 EEG). Durch das EEG sind Netzbetreiber verpflichtet, Erneuerbare-Energien-Anlagen die Einspeisung ihres Stroms in das Netz der allgemeinen Versorgung zu gewährleisten (sog. Einspeisevorrang nach § $11 \mathrm{EEG).} \mathrm{Betreiber} \mathrm{von}$ Anlagen, in denen ausschließlich erneuerbare Energien eingesetzt werden, haben gemäß EEG auf den in diesen Anlagen erzeugten Strom einen Anspruch auf Vergütung (§ 19 Abs. 1 S. 1 EEG). Das EEG unterscheidet dabei zwischen den vier Veräußerungsformen:

(i) Einspeisevergütung nach $\S 21$ Abs. 1 und 2 EEG.

(ii) Marktprämie nach $\S 20$ EEG.

(iii) Mieterstromzuschlag nach $\S 21$ Abs. 3 EEG.

(iv) Sonstige Direktvermarktung nach § 21a EEG.

Anlagenbetreiber sind verpflichtet, jede Anlage mindestens einer dieser Veräußerungsformen zuzuordnen, können allerdings den in ihren Anlagen erzeugten Strom prozentual auf verschiedene Veräußerungsformen aufteilen ( $\$ 21 \mathrm{~b}$ Abs. 2 EEG) sowie die Veräußerungsform zum ersten Kalendertag eines Monats wechseln (§ 21b Abs. 1 EEG).

Die Einspeisevergütung ist ein fixer Betrag, der pro eingespeister $\mathrm{kWh}$ ausbezahlt wird. Nach dem EEG können die Einspeisevergütung Anlagen mit einer Erzeugungsleistung bis zu $100 \mathrm{~kW}$ wählen (§ $21 \mathrm{Abs.} 1$ Nr. 1 EEG). In früheren Fassungen des EEG lag die Grenze höher (vgl. § 37 Abs. 2 EEG i. d. F. v. 21. Juli 2014 bzw. die Regelungen zu Biogas- und Biomethananlagen des EEG i. d. F. v. 28. Juli 2011). Die eingespeiste Jahresarbeit 2018 nach Bundesland ist in Abbildung 2 dargestellt. Es ist ersichtlich, dass diese Veräußerungsform hauptsächlich bei Kleinanlagen genutzt wird, z.B. Photovoltaikanlage auf einem Einfamilienhaus sowie ältere Wind- und Biomasseanlagen [3]. Weiterhin sind die Anlagen auf den süddeutschen Raum konzentriert.

\footnotetext{
${ }^{3}$ Im Hinblick auf die regionale Grünstromkennzeichnung besteht Uneinigkeit, ob die gewählte Regionalitätsdefinition sinnvoll ist, siehe z.B. [14] und [24].
} 


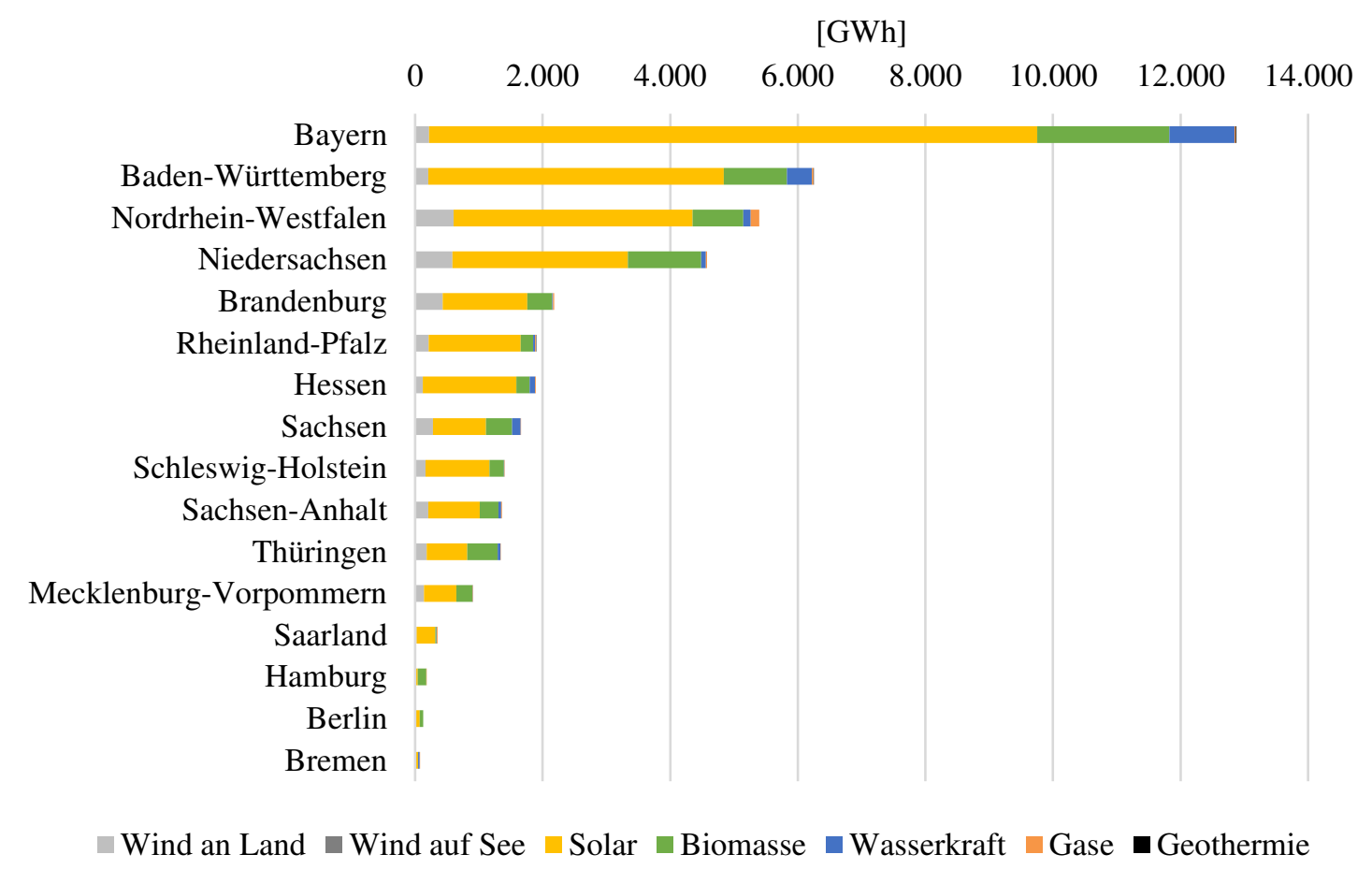

Abbildung 2: $\quad$ Eingespeiste Arbeit im Jahr 2018 in der Veräußerungsform der Einspeisevergütung (Quelle: Eigene Darstellung mit Daten aus [25])

Bei der Veräußerungsform der Marktprämie vermarktet der Betreiber den in seiner Anlage erzeugten Strom selbst oder greift auf einen Dienstleister zurück [26, S. 17-20]. Diese Veräußerungsform ist für Anlagen ab einer gewissen installierten Leistung verpflichtend. Diese beträgt $100 \mathrm{~kW}$ (§ 21 Abs. $1 \mathrm{Nr} .1 \mathrm{EEG})$, lag in früheren Fassungen des EEG jedoch ebenfalls höher. Die Marktprämie wird nach dem EEG 2017 ab einer energieträgerspezifischen Grenze der Anlagenleistung für Windenergieanlagen an Land, Solaranlagen ${ }^{4}$, Biomasseanlagen und Windenergieanlagen auf See über Ausschreibungen bestimmt ( 22 Abs. 1 EEG). Alle Anlagen unter dieser Grenze haben ebenfalls einen Anspruch auf die Marktprämie, deren Höhe sich jedoch unmittelbar aus dem Gesetz ergibt. Betreiber von Anlagen, deren Anlagenleistung diese Grenze übersteigt, bieten mit dem sog. anzulegenden Wert in Cent pro kWh für ihre Anlage. Wenn es zu einem Zuschlag kommt, berechnet sich aus dem anzulegenden Wert die Marktprämie. Dabei wird von dem anzulegenden Wert der Monatsmarktwert des Stroms (Anlage 1 zu § 23a EEG) subtrahiert. Die nach Bundesland 2018 eingespeiste Jahresarbeit in der Veräußerungsform der Marktprämie ist in Abbildung 3 dargestellt. Es ergibt sich ein ggü. der Einspeisevergütung umgekehrtes Bild: Die Erzeugungsmengen sind auf den norddeutschen Raum konzentriert, mit Windenergie und Biomasse als dominierende Erzeugungstechnologien. Die Erzeugungsmengen sind in Summe mehr als drei Mal so hoch als bei der Einspeisevergütung.

\footnotetext{
${ }^{4}$ Im EEG ist unter dem Begriff „Solaranlage“ jede Anlage zur Erzeugung von Strom aus solarer Strahlungsenergie zu verstehen (§ 3 Nr. 41 EEG). Der Begriff „Photovoltaikanlage“ wird fortan synonym verwendet.
} 


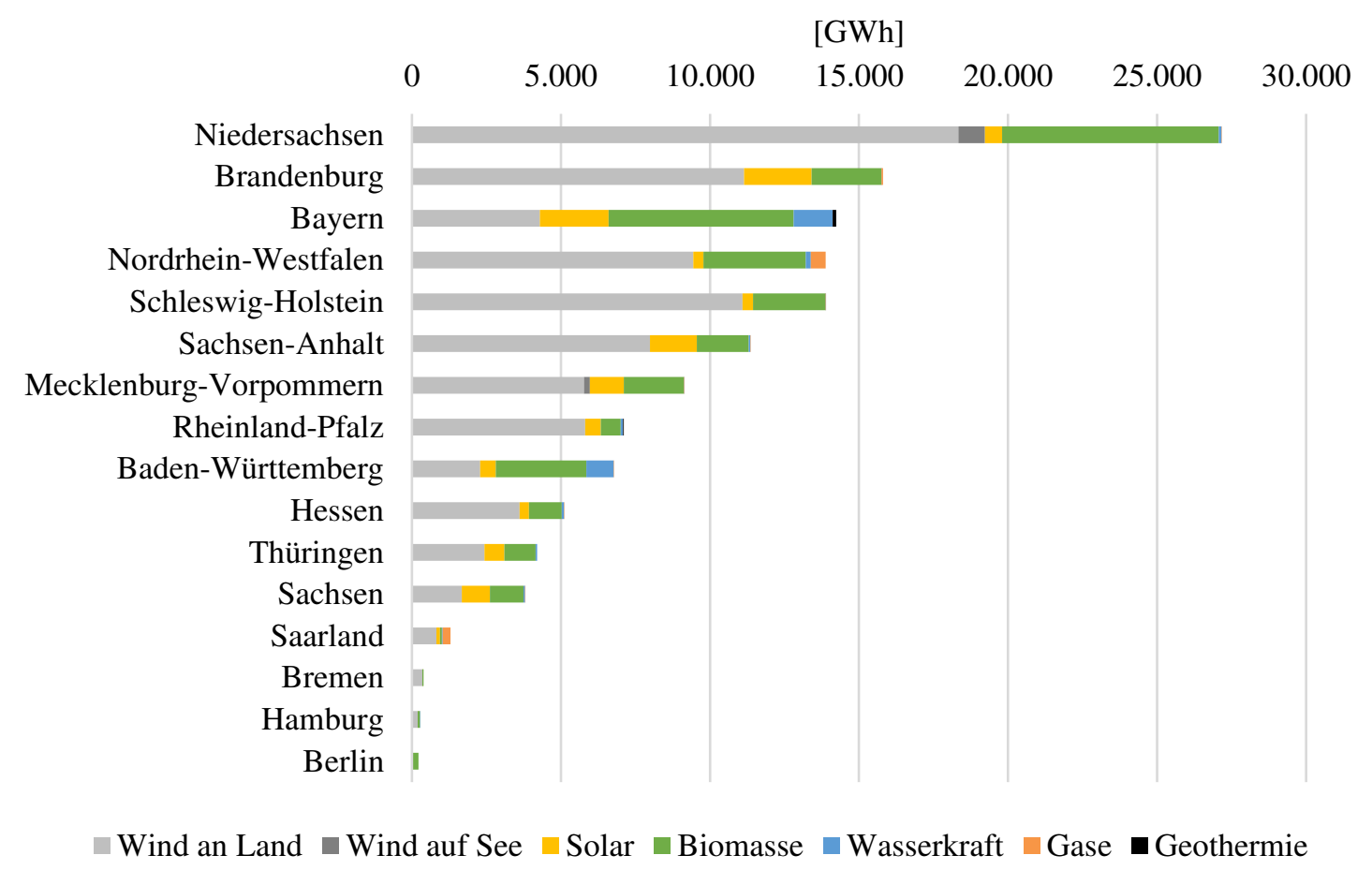

Abbildung 3: Eingespeiste Arbeit im Jahr 2018 in der Veräußerungsform der Marktprämie (Quelle: Eigene Darstellung mit Daten aus [25])

Anlagen, die vor der Einführung des EEG gebaut wurden, nun jedoch von diesem erfasst sind, deren Förderung ausgelaufen ist oder Anlagenbetreibern, die freiwillig auf EEG gestützte Fördermaßnahmen verzichten, bleibt als Veräußerungsform die sogenannte sonstige Direktvermarktung (§ 21a EEG). Dabei wird keine Zahlung nach § 19 oder § 50 EEG in Anspruch genommen. Da diese Veräußerungsform mit höheren finanziellen Risiken verbunden ist, wird nur in wenigen Fällen auf die EEG gestützten Fördermaßnahmen verzichtet [14, S. 38, 27]. Dieser Umstand spiegelt sich in Abbildung 4 wider: Der prozentuale Anteil der Jahresarbeit 2018 in der sonstigen Direktvermarktung beträgt lediglich 0,064\% der vom EEG erfassten Gesamterzeugungsmenge. 


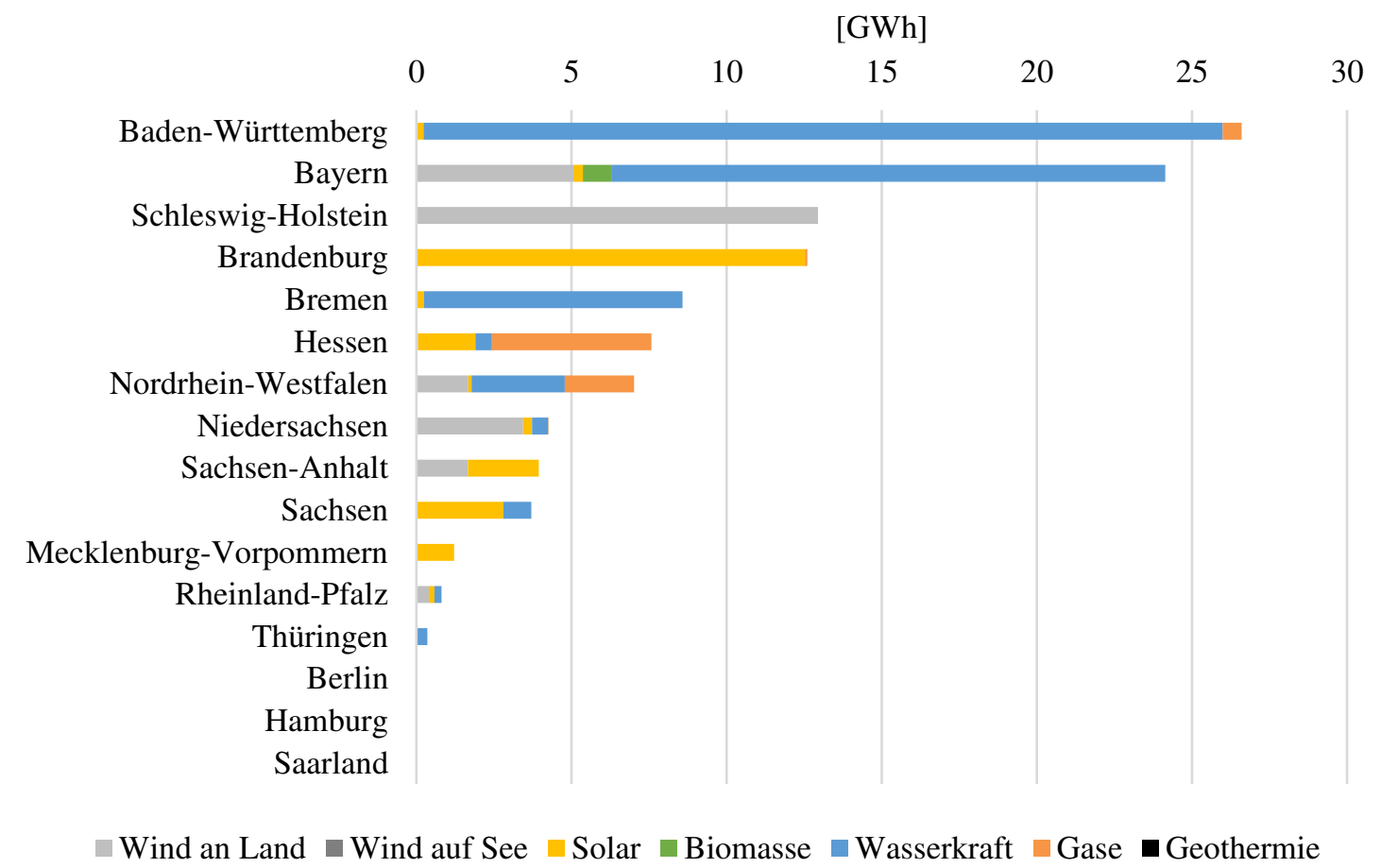

Abbildung 4: Eingespeiste Arbeit im Jahr 2018 in der Veräußerungsform der sonstigen Direktvermarktung (Quelle: Eigene Darstellung mit Daten aus [25])

Bei der vierten Veräußerungsform des Mieterstromzuschlags handelt es sich um eine Sonderform der EEGFörderung für Strom aus Solaranlagen [28]. Für den weiteren Verlauf der Arbeit sind nur die Veräußerungsformen (ii) und (iii) von Relevanz.

\subsection{Grünstromkennzeichnung}

Das Doppelvermarktungsverbot des § 80 EEG verbietet es, den aus geförderten Erneuerbare-Energien-Anlagen stammenden Strom als Ökostrom ${ }^{5}$ zu vermarkten. ${ }^{6}$ Folglich können nur Herkunftsnachweise (HKN) genutzt werden, um gegenüber Letztverbrauchern im Rahmen der Stromkennzeichnung nach § 42 Abs. 1 Nr. 1 Energiewirtschaftsgesetz (EnWG) nachzuweisen, dass ein bestimmter Anteil oder eine bestimmte Menge des Stroms aus erneuerbaren Energien erzeugt wurde ( 3 Nr. 29 EEG). ${ }^{7}$ Herkunftsnachweise werden jeweils für eine erzeugte und an Letztverbraucher gelieferte Strommenge von einer Megawattstunde

\footnotetext{
${ }^{5}$ Die Begriffe „Ökostrom“ oder „Grünstrom“ sind in Deutschland nicht eindeutig definiert. Im Folgenden wird darunter ein Stromprodukt verstanden, welches ausschließlich erneuerbare Energien ausweist [1, S. 84].

${ }^{6}$ Das Doppelvermarktungsverbot des $\$ 80$ EEG richtet sich zwar nur gegen den Anlagenbetreiber und hat für diesen weitreichende Folgen (§ 52 Abs. 2 Satz 1 Nr. 5 i. V. m. Satz 2, § 86 Abs. 1 Nr. 1 EEG). Dennoch kann seitens eines EVU ein Verstoß gegen $§ 5$ UWG vorliegen, wenn es ggü. dem Kunden mit der Herkunft einer Stromlieferung wirbt, ohne über einen entsprechenden Nachweis der Qualität zu verfügen. § 80 Abs. 2 EEG umfasst dabei nicht nur RN und HKN, sondern auch sonstige Nachweise, die die Herkunft des Stroms belegen. Weitere Ausführungen hierzu sind in [15, 26, 27] zu finden.

${ }^{7}$ Nach $\S 31$ KWK-G können auch hocheffiziente KWK-Anlagen beim Bundesamt für Wirtschaft und Ausfuhrkontrolle (BAFA) die Ausstellung von HKN beantragen. Deren Erzeugung kann jedoch nicht im Rahmen der Stromkennzeichnung ausgewiesen werden, da $§ 42$ Abs. 1 Nr. 1 EnWG die KWK nicht als separaten Energieträger aufführt. Europarechtlich wird eine Ausweisung der KWK im Rahmen der Stromkennzeichnung nicht vorgegeben. Der Referentenentwurf des EEG 2020 [29] und den damit verbundenen
} 
ausgestellt ( $\$ 79$ Abs. 5 EEG) und bescheinigen unter anderem, in welcher Art von Anlage ( 79 Abs. 6 Nr. 3 EEG) und mittels welcher Energieträger ( 879 Abs. 6 Nr. 4 EEG) Strom produziert wurde. HKN haben eine Gültigkeit von zwölf Kalendermonaten nach dem Ende des Erzeugungszeitraums (§ 34 HkRNDV). ${ }^{8}$ Um HKN ausstellen zu können, muss die Erzeugungsanlage der Veräußerungsform der sonstigen Direktvermarktung ( $\$ 21 \mathrm{a}$ EEG) zugeordnet sowie im Herkunftsnachweisregister (HKNR) registriert werden. Für die Registrierung, Kontenführung sowie Nutzung des HKNR werden Gebühren erhoben, die in Anlage 1 zu $§ 1$ Abs. 1 HkRNDV aufgeführt sind.

Die auf der EU-Richtlinie 2009/28/EG ${ }^{9}$ basierenden Herkunftsnachweise sind bzgl. ihrer Herkunft nicht auf Deutschland beschränkt, sondern können auch aus weiteren Ländern innerhalb der Europäischen Union (EU) stammen [30]. Über die Anerkennung ausländischer Herkunftsnachweise aus ausgewählten Ländern hat das UBA 2014 ein Rechtsgutachten veröffentlicht [31]. Die Anerkennung von Herkunftsnachweisen aus weiteren Ländern ist jedoch nicht ausgeschlossen und unterliegt der Einzelfallprüfung [32]. Die bisherige Praxis von EU-Ländern, selbst über die Anerkennung von HKN aus Drittstaaten zu entscheiden, soll mit der EEG-Novelle 2021 [29] abgeschafft werden. Bereits 2014 hat der Europäische Gerichtshof (EuGH) in einem Urtei $1^{10}$ klargestellt, dass EU-Mitgliedsstaaten nicht über die notwendige Kompetenz verfügen, über die Anerkennung von HKN aus Drittstaaten zu entscheiden. Zukünftig können demnach nur noch HKN aus Drittstaaten anerkannt werden, die ein entsprechendes Abkommen mit der EU haben. Ein solches Abkommen ist bspw. mit der Schweiz noch nicht geschlossen worden.

Herkunftsnachweise setzen keine Kopplung an die Stromlieferung voraus, wenngleich eine Kopplung auf Antrag des Anlagenbetreibers möglich ist. Mit dieser sog. „optionalen Kopplung“ nach § 16 Abs. 3 HkRNDV wird bestätigt, dass ein EVU den mittels HKN ausgewiesenen Strom tatsächlich bezieht. Eine Zeitgleichheit auf Viertelstundenbasis zwischen Erzeugung und Verbrauch wird nicht vorausgesetzt. ${ }^{11}$ Die optionale Kopplung spielt bei Betrachtung des gesamtdeutschen Ökostrommarktes jedoch eine zu vernachlässigende Rolle [1, S. 204]. Insbesondere tragen regional unterschiedliche Angebots- und Nachfrageverhältnisse [30] zu einer Entkopplung von bilanziellen Herkunftsnachweisen und Energielieferungen und damit zu einem europäischen Handel bei [33]. Durch die Möglichkeit des länderübergreifenden Handels von Herkunftsnachweisen variieren die Preise in Abhängigkeit der Qualität, u.a. nach Herkunftsland, Erzeugungstechnologie und Alter der Anlage [1, S. 26]. Im Vergleich zum Börsenstrompreis ist der Preis für Herkunftsnachweise mit unter 4 Euro pro MWh für HKN aus der Schweiz und unter 1 Euro pro MWh für HKN aus skandinavischen Altanlagen (Stand 08.03.2018 aus [1, S. 214]) jedoch als gering einzustufen [34, S. 121 ${ }^{12}$, wenngleich während der letzten Jahre steigend [1, S. 26]. Da der Handel von HKN üblicherweise bilateral zwischen den Handelspartnern oder über Broker stattfindet, ist die Preistransparenz entsprechend eingeschränkt [1, S. 26].

Möchte ein Energieversorgungsunternehmen ggü. dem Letztverbraucher nachweisen, dass 100\% der gelieferten Strommenge aus erneuerbaren Energien stammen, so muss es HKN in gleicher Höhe entwerten [35, S. 33-34]. Wird auf einen vollständigen Nachweis der Grünstromeigenschaft mittels HKN verzichtet, sind

Änderungen des KWK-G sieht vor, dass zukünftig das UBA HKN für Strom aus erneuerbarer KWK ausstellt, das BAFA weiterhin HKN für hocheffiziente KWK. Jedoch gelten für beide HKN die gleichen Anforderungen hinsichtlich der Eigenschaften (sog. kombinierter Herkunftsnachweis).

${ }^{8}$ Zukünftig soll die Gültigkeit, definiert als Zeitraum zwischen Produktion der Energieeinheit und Entwertung des HKN, 18 Monate betragen. Der bisherige Zeitraum von 12 Monaten für die Übertragung von HKN soll dagegen unverändert bleiben [29].

${ }^{9}$ Richtlinie 2009/28/EG des Europäischen Parlaments und des Rates vom 23. April 2009 zur Förderung der Nutzung von Energie aus erneuerbaren Quellen und zur Änderung und anschließenden Aufhebung der Richtlinien 2001/77/EG und 2003/30/EG.

${ }^{10}$ EuGH, Urteil Green Network SpA, C-66/13, ECLI:EU:C:2014:2399.

${ }^{11}$ Das Bilanzkreismanagement für Strom erfolgt auf viertelstündlicher Basis.

${ }^{12}$ Dies gilt i.d.R. nicht für HKN aus Anlagen, die einen Förderanspruch nach dem EEG besitzen und freiwillig in die sonstige Direktvermarktung nach § 21a EEG wechseln. Diese Anlagen werden aus der EEG-Förderung „,heraus gekauft“. Daher ist meist nur eine Beimischung von $\mathrm{HKN}$ aus diesen Anlagen (z.B. 10\%) wirtschaftlich darstellbar [27, S. 113]. 
die Erzeugungstechnologien bei der Ausweisung des EEG-Anteils anteilig zu kürzen (§ 78 Abs. 4 EEG). Der überwiegende Anteil der heute in Deutschland angebotenen Ökostromtarife basiert auf ausländischen Herkunftsnachweisen, insbesondere aus Skandinavien und dem Alpenraum [24, 36, 37]. Über 90\% davon stammen aus Wasserkraft, wenngleich ein leichter Trend zu weiteren Erzeugungstechnologien, insbesondere Wind und Biomasse, zu beobachten ist [1, S. 26-27].

\subsection{Regionale Grünstromkennzeichnung}

Mit der EEG-Novelle 2014 wurde das „Grünstromprivileg“ ersatzlos gestrichen, welches es Stromlieferanten ermöglichte, sich von der Zahlung der EEG-Umlage ganz oder teilweise befreien zu lassen, auf direktvermarkteten Strom zurückzugreifen und diesen Strom in der Stromkennzeichnung als Ökostrom auszuweisen, wenn gewisse Anforderungen an die Stromlieferung an Letztverbraucher erfüllt wurden ( $\$ 39$ Abs. 1 EEG i. d. F. v. 28. Juli 2011). Vor diesem Hintergrund und aufgrund des steigenden Drucks durch Branchenvertreter, alternative Vermarktungswege für EEG-geförderten Strom zu ermöglichen, wurde mit dem EEG 2017 die regionale Grünstromkennzeichnung eingeführt [24, 38, 39]. ${ }^{13}$ Seit Anfang 2019 existiert beim UBA infolgedessen das Regionalnachweisregister [40]. Das RNR ist - im Gegensatz zum HKNR nicht im Unionsrecht begründet, d.h. das RNR geht über die EU-Richtlinie 2009/28/EG hinaus [15, S. 35].

Das Regionalnachweisregister des Umweltbundesamts ermöglicht es, mittels Regionalnachweisen im Rahmen der Stromkennzeichnung nach $\S 42$ EnWG gegenüber einem Letztverbraucher die regionale Herkunft eines bestimmten Anteils oder einer bestimmten Menge des verbrauchten Stroms aus erneuerbaren Energien auszuweisen ( $33 \mathrm{Nr}$. 38 EEG). Zusätzlich können Anlagen, die RN ausstellen, konkret benannt werden [5, 24, 26, S. 64, 27, 38]. Mittels Herkunftsnachweisen können Energieversorgungsunternehmen zwar ebenfalls mit der Regionalität einer Stromlieferung werben, dürfen diese jedoch nicht in der Stromkennzeichnung ausweisen [39, S. 305]. ${ }^{14} \mathrm{RN}$ werden jeweils für eine erzeugte und an Letztverbraucher gelieferte Strommenge von einer Kilowattstunde ausgestellt (§ 79a Abs. 5 S. 1 EEG) und verfallen, wenn sie nicht spätestens 24 Kalendermonate nach dem Ende des Erzeugungszeitraums entwertet worden sind ( $\$ 35$ HkRNDV). Sie besitzen damit eine doppelt so lange Gültigkeit wie HKN (siehe Kapitel 2.3). Für die Ausstellung von RN kann auf nach dem EEG geförderte Anlagen zurückgegriffen werden, welche sich in der Veräußerungsform der Marktprämie ( $\$ 20$ EEG) befinden, ohne dabei gegen das Doppelvermarktungsverbot des $\S 80 \mathrm{EEG}$ zu verstoßen. Anlagen in anderen Veräußerungsformen können das RNR nicht nutzen. Damit möchte der Gesetzgeber vermeiden, dass bereits vor der Einführung des RNR existierende Regionalstromprodukte durch dessen Einführung tangiert werden [39]. Folglich erweitert das RNR den Pool möglicher Anlagen, der für die Umsetzung eines Regionalstromprodukts genutzt werden kann, um Anlagen, die sich in der EEG-Förderung befinden (siehe Kapitel 2.5). ${ }^{15}$

Wurde der anzulegende Wert einer Anlage, aus der sich die Marktprämie ableitet, gesetzlich bestimmt (siehe Kapitel 2.2), so reduziert sich dieser Wert um 0,1 Cent pro Kilowattstunde für den Strom, für den dem Anlagenbetreiber ein RN ausgestellt worden ist (§ 53b EEG). In der Gesetzesbegründung zum EEG 2017 [41] wird dies mit der (bereits europarechtlich erforderlichen) Vermeidung von Überförderung begründet, weshalb die Regelung nicht für Anlagen gilt, deren anzulegender Wert über Ausschreibungen be-

\footnotetext{
${ }^{13} \mathrm{Zu}$ den Gründen für die Abschaffung des Grünstromprivilegs und den diskutierten Alternativen der regionalen Grünstromkennzeichnung siehe [37].

${ }^{14}$ Für zulässige Vermarktungsvarianten regionaler Stromprodukte siehe Kapitel 2.5 sowie [5].

${ }^{15}$ Im Jahr 2018 stammten 78,20\% der eingespeisten Jahresarbeit aus Anlagen in der Veräußerungsform der Marktprämie, 21,74\% aus Anlagen in der Einspeisevergütung und 0,06\% aus der sonstigen Direktvermarktung. Diese Zahlen umfassen jedoch ausschließlich Anlagen, die einen Zahlungsanspruch nach dem EEG besitzen [25].
} 
stimmt wurde. Ob Anlagenbetreiber zukünftige, unsichere Erlöse durch den Verkauf von Regionalnachweisen in ihre Gebote tatsächlich miteinbeziehen, bleibt jedoch anzuzweifeln [24, 26, S. 48, 38, 39]. RN können auch von Erzeugungsanlagen außerhalb des Bundesgebiets ausgestellt werden, wenn die entsprechende Anlage einen Zuschlag in einer Ausschreibung nach § 5 Abs. 2 Satz 2 EEG erhalten hat ( $\$ 23$ Abs. 3 Nr. 3 HkRNDV) und sich in einer nach dem RNR definierten Region befindet ( $\$ 23$ Abs. 3 Nr. 1 HkRNDV). Dabei ist bei einer Registrierung von Biomasseanlagen $\S 23$ Abs. 3 Nr. 2 HkRNDV zu berücksichtigen, der eine Verwendung anderer Energieträger ausschließt.

Für die Registrierung, Kontenführung sowie Nutzung des Regionalnachweisregisters sind Gebühren zu entrichten, die in Anlage 2 zu § 1 Abs. 2 HkRNDV aufgeführt sind. Regionalnachweise dürfen nur entlang der vertraglichen Lieferkette des Stroms, für den sie ausgestellt worden sind, übertragen werden ( $79 \mathrm{a}$ Abs. 5 EEG). Dies soll die Glaubwürdigkeit eines auf Regionalnachweisen basierenden Regionalstromprodukts erhöhen [39]. Dennoch unterscheidet sich die Kopplung des RNR von der „optionalen Kopplung“ des HKNR. Letztere setzt eine Lieferung der Strommenge in den Bilanzkreis des Energieversorgungsunternehmens voraus, an welches der HKN übertragen wird ( $\$ 16$ Abs. 3 HkRNDV). Demgegenüber verlangt $\S 79 \mathrm{a}$ Abs. 5 EEG lediglich, dass ein Stromliefervertrag, d.h. ein privatrechtliches Verpflichtungsgeschäft, vorliegt [39]. Infolgedessen können Regionalnachweise zusammen mit der vertraglich festgelegten Strommenge beliebig oft verkauft werden [14, 26, S. 38]. Somit besteht die Möglichkeit, die Kopplung der RN an die Stromlieferung, bspw. durch einen gegenläufigen Stromliefervertrag, zu umgehen [39]. Es gilt jedoch zu beachten, dass gegenläufige Stromlieferverträge auch ungewollt während des Handels auftreten können, ohne dass diese zum Ziel haben, die Kopplung zu umgehen [16, S. 6]. Eine Zeitgleichheit zwischen Stromlieferung und Verbrauch setzt das RNR, ebenso wie das HKNR, nicht voraus. Dennoch ergeben sich durch die Intention des Gesetzgebers, keinen freien Handel von RN zu ermöglichen, einige Anforderungen an korrespondierende Stromlieferverträge. Zu diesen zählen u.a. die Zuordenbarkeit von Strommengen zu Vertragspartnern, was nur bei OTC-Geschäften ${ }^{16}$ sicherzustellen ist, der Ausschluss von Rahmenverträgen als Stromlieferverträge, der Ausschluss von nachträglichen Lieferverträgen sowie die Kongruenz der vertraglichen Liefermengen mit den Lieferzeiträumen der Regionalnachweise [26, S. 39-42].

Der Regionalanteil ist auf den in der Stromkennzeichnung ausgewiesenen EEG-Anteil begrenzt $(\S 42$ Abs. 1 EnWG), der sich zwischen privilegierten und nicht privilegierten Letztverbrauchern unterscheidet, je nach Höhe der abgeführten EEG-Umlage pro Kilowattstunde ( $§ 78$ Abs. 5, 63 ff. EEG). Zu den privilegierten Letztverbrauchern zählen in der Regel Industriebetriebe, die eine hohe Stromkostenintensität aufweisen, während Abnehmer ohne Privileg typischerweise Kleinverbraucher wie Haushaltskunden sind. Für letztere liegt der EEG-Anteil, der die Obergrenze für den ausweisbaren Regionalanteil bildet, momentan bei 55,61\% (Stand 2020 für das Bezugsjahr 2018). Aufgrund dieser Begrenzung auf den EEG-Anteil darf gegenüber dem Endkunden seitens eines EVU nicht der Eindruck erweckt werden, 100\% der gelieferten Strommenge stammten aus einer bestimmten Anlage [39] oder stammten aus der nach § 79a EEG definierten Region, wenn der Nachweis der Regionalität ausschließlich mittels RN erfolgt. ${ }^{17}$

Ein Ziel des Gesetzgebers war es unter anderem, mit der Einführung des Regionalnachweisregisters die Akzeptanz von Neuanlagen zu erhöhen, indem Strom konkreter Anlagen vor Ort vermarktet und somit die Akzeptanz durch die lokale Bevölkerung erhöht wird [41, S. 243, 43]. Mit dem RNR eröffnet sich für Versorger somit die Möglichkeit, ihren Kunden einen Stromtarif anzubieten, der die Regionalität des Stroms explizit ausweist. Mit diesem Ansatz der Regionalstromkennzeichnung wurde das bisherige Prinzip

\footnotetext{
${ }^{16}$ Der OTC-Handel (englisch: Over-The-Counter; deutsch: über den Tresen) beschreibt den außerbörslichen Handel, der entweder bilateral zwischen den Handelspartnern oder über Broker stattfindet. [42, S. 19-20].

${ }^{17}$ Für eine weitere Diskussion siehe $[5,15,39]$.
} 
der Stromkennzeichnung von EEG-gefördertem Strom durchbrochen, wonach keine Zuordnung von EEGStrom aus einzelnen Anlagen auf einzelne Letztverbraucher erfolgte [43].

Da RN ausschließlich dazu dienen, die Herkunft des Stroms aus erneuerbaren Energien auszuweisen, jedoch nicht die Grünstromeigenschaft, müssen bei regionalen Grünstromprodukten unter Nutzung des RNR zusätzlich zu den Regionalnachweisen Herkunftsnachweise in Höhe der gelieferten Strommenge entwerten werden [39]. Dies ist jedoch optional, da ein Nachweis der Regionalität des Stroms keinen Nachweis der Grünstromeigenschaft voraussetzt und umgekehrt. Folglich muss ein EVU, das einen regionalen Grünstromtarif unter Nutzung des RNR anbietet, aktuell Nachweise in Höhe von 156,61 Prozent der gelieferten Strommenge beschaffen. Diese 156,61 Prozent setzen sich aus 100 Prozent HKN und 56,61 Prozent RN zusammen.

Möchte ein EVU ein regionales Grünstromprodukt unter Nutzung des RNR im Rahmen der Stromkennzeichnung nach § 42 EnWG ausweisen, so muss es im ersten Schritt den Strommix für das entsprechende Stromprodukt ermitteln [35, S. 16-18]. Danach erfolgt im zweiten Schritt die Ausweisung der Grünstromeigenschaft mittels HKN [35, S. 33-34]. Wird auf eine vollständige Grünstellung des Strommixes mittels HKN verzichtet, werden bei der Ausweisung des EEG-Anteils die relativen Anteile der Erzeugungstechnologien anteilig gekürzt ( $\$ 78$ Abs. 4 EEG). Im dritten Schritt wird der EEG-Anteil dargestellt, auf dessen Ausweisung der Letztverbraucher aufgrund der Zahlung der EEG-Umlage ein Anrecht hat ( 78 Abs. 1 EEG).$^{18}$ Der vierte Schritt besteht in der Regionalisierung des EEG-Anteils mittels Regionalnachweisen [35, S. 35]. Unter der Annahme, dass der Letztverbraucher sämtlich Umlage- und Nachweiskosten zu tragen hat, ergeben sich für diesen in Summe Nachweis- und Umlagekosten in Höhe von 211,22 Prozent der gelieferten Strommenge. ${ }^{19}$

\subsection{Vermarktungsvarianten regionaler Stromprodukte}

Nachfolgend wird, basierend auf [14] und mittels Ergänzungen aus [26] und [17], zwischen fünf Optionen der Regionalstromvermarktung unterschieden (siehe Abbildung 5). Bei den Optionen 1 bis 4 handelt es sich um Grünstromprodukte, die in der Stromkennzeichnung zzgl. zum EEG-Anteil ausschließlich erneuerbare Energien ausweisen und auf unterschiedliche Weise einen Bezug zur Region herstellen. Bei Option 5 handelt es sich um keine Grünstrom-, sondern Graustromprodukte ${ }^{20}$. Der Regionalitätsbezug wird entweder über Regionalnachweise, den Stromeinkauf oder eine Kombination hergestellt. Jedoch kann die Regionalität im Rahmen der Stromkennzeichnung nur mittels RN ausgewiesen werden (siehe Kapitel 2.4). Werbeaussagen hinsichtlich Regionalität sind somit von der Ausweisung der Regionalität zu unterscheiden (siehe Abbildung 6).

\footnotetext{
${ }^{18}$ Die Ausweisung des EEG-Anteils gegenüber dem Letztverbraucher in Abhängigkeit der Höhe der gezahlten EEG-Umlage wird oft kritisiert, da hierdurch der Eindruck erweckt werde, ein EVU sei grüner als es in Wirklichkeit ist [24].

${ }^{19}$ Die 211,22\% der ausgewiesenen Strommenge ergeben sich aus 100\% Herkunftsnachweisen, 55,61\% EEG-Umlage und 55,61\% Regionalnachweisen. Die Kosten für die Regional- und Herkunftsnachweise beinhalten neben den Zertifikatskosten ggf. noch weitere Kosten, wie bspw. für operative Aufwände und Margen.

${ }^{20}$ Unter Graustrom werden Stromlieferungen unbekannter Herkunft verstanden [44, S. 225] oder auch Stromlieferungen, die nicht mittels Herkunftsnachweisen unterlegt sind [45, S. 232]. Umgangssprachlich ist im Kontext von Stromlieferverträgen auch die Bezeichnung „,konventioneller Strommix“ gebräuchlich, wenngleich der (ausgewiesene) Strommix meist nicht ausschließlich aus konventionellen Erzeugungsanlagen stammt.
} 


\section{Option 1: $\quad$ Keine Vermarktung regional erzeugten Stroms aus erneuerbaren Energien}

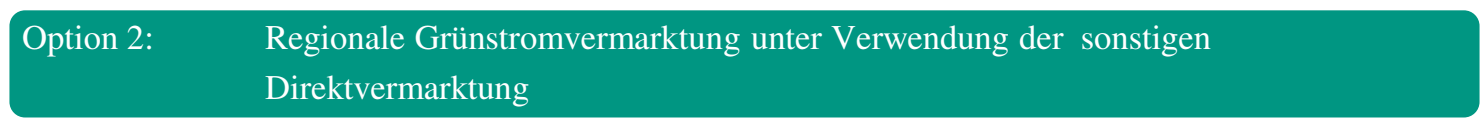

\section{Option 3: $\quad$ Regionale Grünstromvermarktung unter Verwendung der Marktprämienvermarktung}

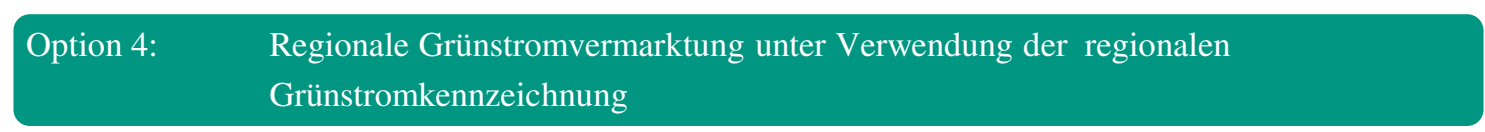

\section{Option 5: $\quad$ Regionale Graustromvermarktung}

Abbildung 5: Vermarktungsvarianten regionaler Stromprodukte (Quelle: Eigene Darstellung in Anlehnung an [14, 26])

Bei Option 1 erfolgt keine Vermarktung regional erzeugten Stroms aus erneuerbaren Energien, sondern ein Bezug zur Region wird lediglich über den Produktnamen und/oder Reinvestitionen in die Region hergestellt. Dagegen erfolgt bei Option 2 entweder eine börsliche Beschaffung des Stroms oder der Strom wird OTC beschafft, jeweils zzgl. HKN aus der Region. Alternativ erfolgt eine OTC-Beschaffung aus der Region zzgl. HKN beliebigen Ursprungs. Wichtig dabei ist, dass die Regionalität auf mindestens eine Weise sichergestellt wird, d.h. über eine nachverfolgbare Lieferkette der regionalen Strombeschaffung oder über HKN aus der Region [vgl. 5]. Die dritte Option sieht eine Beschaffung regional erzeugten Stroms aus geförderten Anlagen vor, die sich in der Marktprämie befinden. Eine Grünstellung erfolgt mittels HKN beliebigen Ursprungs. Der Bezug zur Region wird nicht über die Nennung konkreter Anlagen, sondern durch unspezifische Begriffe geschaffen, wie bspw. ,aus der Region“. ${ }^{21}$ Bei den Optionen 1, 2 und 3 ist die Stromkennzeichnung identisch (siehe Abbildung 6c).

Bei der vierten Option wird direktvermarkteter Strom aus Anlagen, die sich in der nach § 79a Abs. 6 EEG definierten Region befinden, zusammen mit RN verwendet. Weiterhin werden für den Nachweis der Grünstromeigenschaft HKN genutzt. Da mittels RN lediglich die Eigenschaft der Regionalität in Höhe des EEG-Anteils nachgewiesen werden kann, muss für den verbleibenden Anteil der Strommenge ein anderer Nachweis der Regionalität erbracht werden, sofern mit einem 100\% regionalen Ökostromprodukt geworben werden soll [5]. Allerdings spricht laut Lehnert und Rühr [5] vieles dafür, dass für diesen verbleibenden Anteil die Region nicht mehr durch § 79a Abs. 6 EEG vorgegeben ist. Weiterhin sei davon auszugehen, dass der Nachweis der Regionalität nicht durch HKN erbracht werden müsse, sondern eine nachverfolgbare Lieferkette ausreiche (siehe Option 2). Die Stromkennzeichnung dieser Vermarktungsvariante ist in Abbildung $6 \mathrm{~d}$ dargestellt.

\footnotetext{
${ }^{21}$ Es herrscht Uneinigkeit darüber, ob diese Vermarktungsform nach Einführung des RNR noch zulässig ist oder einen Verstoß gegen das Doppelvermarktungsverbot des § 80 EEG darstellt. Lehnert und Sösemann [27] argumentieren, dass kein Verstoß vorliegt, solange durch die Regionalität die Erzeugungstechnologie nicht identifiziert werden kann und für den Kunden erkennbar ist, dass der Ort der Erzeugung ein zusätzliches Produktmerkmal neben der Erzeugungstechnologie ist. Nach Maaß et al. [26] handelt es sich zumindest um eine rechtliche Grauzone, wobei Lehnert et al. [15] und Lehnert und Rühr [5] die Auffassung vertreten, dass durch die Einführung des RNR nunmehr eine höhere Anzahl an Argumenten für eine unzulässige Vermarktung spricht als dagegen. Im Allgemeinen ist es jedoch schwierig, Verstöße gegen das Doppelvermarktungsverbot nachzuweisen [26, S. 21].
} 
Bei den vier zuvor beschriebenen Optionen können die relativen Anteile an über die Börse beschafften Stroms, mittels OTC beschafften konventionellen Stroms, Marktprämienstroms sowie Stroms aus der sonstigen Direktvermarktung variieren. Gleiches gilt für die relativen Anteile an Regionalnachweisen und Herkunftsnachweisen. Bei Letzteren kann zudem die „optionale Kopplung“ des HKNR genutzt werden.

Neben der Vermarktung grünen Stroms ist auch eine regionale Graustromvermarktung denkbar (Option 5). Abbildung 6a stellt die Stromkennzeichnung dieser Vermarktungsvariante dar. Allerdings scheint es momentan keine Stromtarife zu geben, die mit der regionalen Erzeugung, bspw. aus Kohle- oder Atomkraftwerken, werben [26, S. 14]. Lediglich Stromtarife, die den Bezug zur Region über den Produktnamen oder über die Art des Anbieters (z.B. Stadtwerk) herstellen, werden angeboten. Es sind also ähnliche Produkte wie bei Option 1, nur der Strommix ist nicht mit HKN unterlegt. Eine Kombination von Graustrom bekannter oder unbekannter Herkunft mit Regionalnachweisen ist ebenfalls möglich (siehe Abbildung 6b). Die praktische Relevanz eines solchen Regionalstromprodukts wird jedoch angezweifelt [14, S. 47].

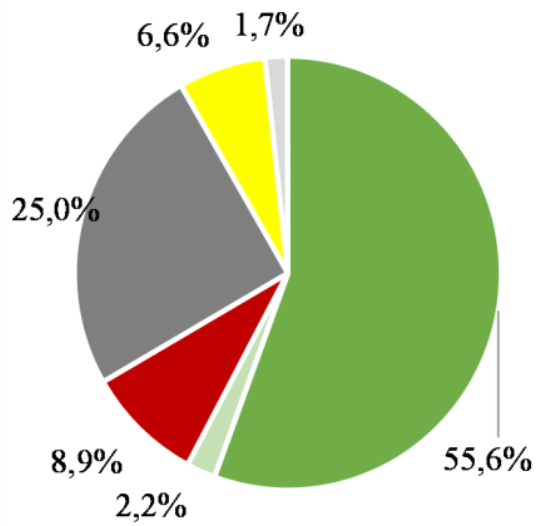

(a) Konventioneller Stromtarif

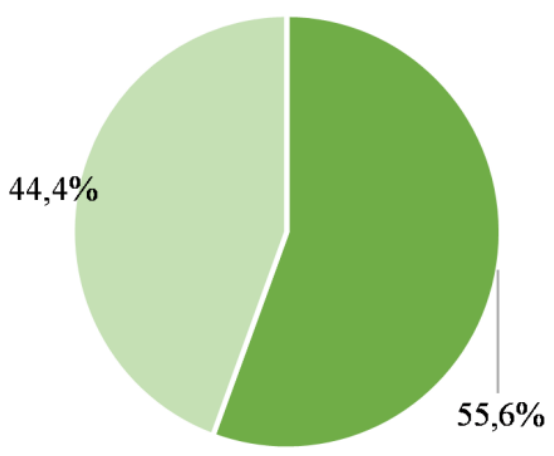

(c) Ökostromtarif

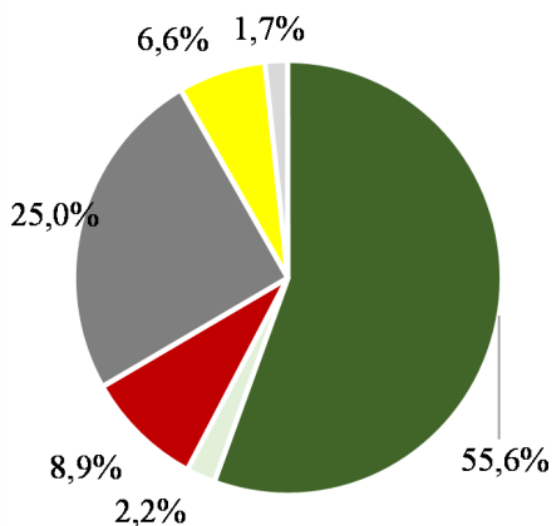

(b) Regionalstromtarif

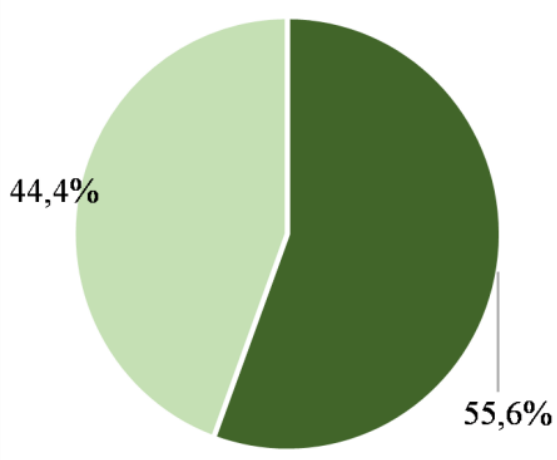

(d) Regionaler Ökostromtarif
- Kernkraft

Erdgas

Erneuerbare Energien, finanziert aus der EEG-Umlage

Sonstige erneuerbare Energien
Eohle

Sonstige fossile Energieträger

In regionalem Zusammenhang zum Stromverbrauch erzeugt

Abbildung 6: Exemplarische Stromkennzeichnung ohne und mit Nutzung des Regionalnachweisregisters 
Die vielfältigen Möglichkeiten für Energieversorgungsunternehmen, Regionalstromprodukte zu vermarkten, erschwert die Nachvollziehbarkeit für den Verbraucher und reduziert die Transparenz [16, 24, 38]. Grenzen der Vermarktungsvarianten regionaler Stromprodukte werden neben dem EEG, dem EnWG und dem Zivilrecht, v.a. durch das Wettbewerbsrecht und im Speziellen durch das Lauterkeitsrecht gezogen. Ausführungen zur wettbewerbsrechtlichen Einordnung von Regionalstromprodukten sind in [15], [5] und [26] zu finden. 


\section{Methodik}

Dieses Kapitel erläutert die dieser Arbeit zugrundeliegende Methodik. Zunächst wird auf die Methodenauswahl und im Speziellen auf die Interviewformen eingegangen. Daran schließen Ausführungen zu den Rahmenbedingungen der Interviews, zur Erstellung des Leitfadens, zur Expertenakquise und zur Auswertung der Interviews an.

\subsection{Methodenauswahl}

Die Forschungsfrage dieser Arbeit (siehe Kapitel 1.2) adressiert Marktentwicklungen im Endkundensegment von Energieversorgungsunternehmen als auch jüngste Änderungen in der deutschen Regulatorik. Folglich hat die Forschungsfrage einen starken Fokus auf nationalen Entwicklungen, was zusammen mit der Aktualität des Themas die zur Verfügung stehende Literatur stark einschränkt. Eine reine Literaturrecherche scheidet daher als Methodik aus. Um einen Überblick zum Themenfeld zu erhalten und die gewonnenen Erkenntnisse in weitere Forschungsarbeiten einfließen zu lassen, bieten sich Experteninterviews als qualitative Methode der Sozialforschung an [46, S. 36]. Sie ermöglichen aufgrund ihres explorativen Charakters, wesentliche Sachverhalte zu identifizieren [47, S. 159, 48, S. 23-24] sowie auf Prozesswissen zuzugreifen [48, S. 25, 49, S. 23]. Um sowohl Fakten, Stellungnahmen und Bewertungen zum Themenfeld zu erhalten, stellen semistrukturierte Leitfadeninterviews zur Datenerhebung einen unter Abwägung von Nutzen und Aufwand gangbaren Weg dar [46, S. 37].

\subsection{Interviewformen}

Für die Durchführung von Experteninterviews stehen verschiedene Interviewformen zur Verfügung, wie etwa ein persönliches Interview im direkten Gegenüber als auch ein Telefoninterview oder ein schriftliches Interview. Nachfolgend wird, orientiert an Mayer [46], auf diese drei Möglichkeiten eingegangen. ${ }^{1}$

\subsubsection{Schriftliches Interview}

Schriftliche Befragungen besitzen den Vorteil, dass annähernd zeitgleich eine große Anzahl von Experten befragt werden kann [50, S. 425]. Allerdings kann die Qualität der Ergebnisse leiden, wenn schriftliche Interviews in Form eines Fragebogens allein durch den Interviewten ohne Hilfestellung bzw. Kommentatoren (Interviewer) beantwortet werden, sodass Fragen falsch oder unzureichend bearbeitet werden [46, S. 101]. Außerdem können die äußeren Umstände während des Ausfüllen des Fragebogens nicht kontrolliert werden [50, S. 425]. Folglich ist bei der Erstellung eines schriftlichen Fragebogens deutlich mehr Sorgfalt angebracht als bei einem Leitfaden einer mündlichen Befragung [51, S. 353-354]. Zudem bietet eine mündliche Befragung mehr Spielraum für die spontane Fortentwicklung des Gesprächsverlaufs [50, S. 425].

\footnotetext{
${ }^{1}$ Eine Übersicht mit den Stärken und Schwächen verschiedener Befragungsmethoden ist in [50, S. 425] zu finden.
} 


\subsubsection{Persönliches Interview}

Bei der mündlichen Befragung bzw. dem persönlichen Interview führt der Interviewer das Gespräch und übernimmt Lage-, Regel- und Kontrollfunktion [46, S. 102]. Zu berücksichtigen ist dabei, dass der Interviewer aufgrund seines eigenen persönlichen Auftretens den Interviewten beeinflussen und infolgedessen einen Verzerrungsfaktor darstellen kann [50, S. 425]. Um solche Verzerrungsfaktoren möglichst zu vermeiden, sollte der Interviewer persönliche Stellungnahmen oder andere Einflussnahmen ausschließen und alle Interviews gleich durchführen (siehe Kapitel 3.3) [46, S. 102].

\subsubsection{Telefoninterview}

Das Telefoninterview stellt den Mittelweg zwischen den beiden zuvor genannten Befragungsarten dar. Bei dieser Methode wird der Interviewte nicht durch das Auftreten des Interviewers beeinflusst, jedoch besteht auch hier die Möglichkeit, im Gespräch Rückfragen zu stellen. Nachteilig ist dabei der Umstand, dass der Interviewer beim Telefoninterview im Vergleich zum persönlichen Interview weniger Kontrolle über die Situation besitzt und ihm die Möglichkeit genommen ist, nonverbale Kommunikation wahrzunehmen [48, S. 39]. Zudem ist es nicht möglich, zur Erläuterung komplexer Fragestellungen entsprechendes Demonstrationsmaterial zu verwenden [46, S. 102, 52, S. 153]. Zielen die Interviews vorrangig auf eine Informationsgewinnung ab, kommen diese Nachteile jedoch weniger zum Tragen [48, S. 39]. Ein besonders hervorzuhebender Vorteil bei einer Vielzahl an Interviewpartnern auf großen räumlichen Distanzen ist der geringere Zeit- und Kostenaufwand durch die nicht notwendige Vor-Ort-Präsenz [52, S. 153]. Für ein Telefoninterview kann, ebenso wie beim persönlichen Interview, ein strukturierter Leitfaden entwickelt und als Gesprächsgrundlage genutzt werden, wobei nach Möglichkeit offene und präzise formulierte Frage verwendet werden sollten [53, S. 205].

\subsubsection{Zwischenfazit}

Die Rigidität schriftlicher Interviews führt dazu, dass diese im Rahmen dieser Arbeit nicht eingesetzt wurden. Aufgrund des explorativen Charakters, der Flexibilität bei der Durchführung sowie der Möglichkeit, nonverbale Kommunikation wahrzunehmen, wurden persönliche Interviews als bevorzugte Methodik identifiziert. Bei weit entfernten Interviewpartnern kam jedoch der Vorteil der Zeit- und Kosteneffizienz von Telefoninterviews zum Tragen. Zusätzlich spitzte sich bei der Durchführung der letzten Interviews die Situation der „Coronakrise“ zu [54], sodass bei diesen von persönlichen Interviews abgesehen werden musste.

Die Interviews für diese Arbeit wurden als sogenannte Tandeminterviews durchgeführt, bei denen zwei Interviewer gleichzeitig den einzelnen Interviewten befragen [55, S. 43-44]. Je nach Situation entsteht dadurch in Summe eine höhere Aufmerksamkeit bei den Interviewern gegenüber dem Interviewten und folglich eine lückenlosere Abfrage. Zudem entsteht durch zwei Personen eine gegenseitige Kontrollfunktion bei den Interviewern untereinander, bspw., dass alle Leitfragen sowie für das Vorhaben interessante Nachfragen gestellt werden. Dies erhöht die Qualität und Objektivität und hilft mögliche Fehlerquellen auszuschließen. Für die spätere Auswertung ist es ebenfalls hilfreich auf zwei unabhängige Wahrnehmungen zu dem jeweiligen Interviewten zurückgreifen zu können, um somit die Aussagen objektiver einzuschätzen und zu interpretieren. Hinsichtlich der Gesprächsdynamik sind keine eindeutigen Aussagen möglich. Einerseits kann die Gesprächssituation durch eine dritte Person aufgelockert werden, andererseits kann es auch schwieriger wird, sensible Themen anzusprechen. 


\subsection{Rahmenbedingungen der Interviews}

Zur Bewertung von Interviews können nicht die üblichen Gütemaße der quantitativen Sozialforschung Objektivität, Reliabilität und Validität [56] angewandt werden [48, S. 92, 57, S. 573]. Vielmehr muss die einer jeden Interviewsituation individuell zugrunde liegende Subjektivität berücksichtigt werden [58, S. 683]. Dennoch empfiehlt es sich, bei der Durchführung von Interviews auf möglichst gleiche Rahmenbedingungen zu achten, sowohl hinsichtlich der Fragen (siehe Kapitel 3.4), der Dauer, der äußeren Einflüsse als auch bei der Beziehung zwischen Interviewer und Interviewtem [48, S. 90-91, 57, S. 572-573]. Die Zusicherung von Anonymität hilft, dass sich der Interviewte freier, ungezwungener und zumeist auch informativer bzw. detaillierter zu den einzelnen Fragen äußern kann [46, S. 46, 48, S. 40]. Dies ist insbesondere wichtig, wenn - wie im Rahmen dieser Arbeit - die Gespräche aufgezeichnet werden [52, S. 157]. Tonaufzeichnungen stellen sicher, dass die Konzentration während der Gespräche auf deren Verlauf liegt und nach den Interviews auf alle Informationen des Gesprächs zugegriffen werden kann [46, S. 47, 48, S. 40].

In Anlehnung an Bogner et al. [48] wurden die Interviews in die drei Teile (i) Vorgespräch, (ii) Interview und (iii) Nachgespräch unterteilt. Im Vorgespräch wurden die Rahmenbedingungen erläutert, im Nachgespräch wuden offen gebliebene Fragen beantwortet sowie das weitere Vorgehen erläutert. Die eigentliche Informationsgewinnung fand in Teil (ii) statt.

\subsection{Leitfaden}

Zur Vorbereitung und Vereinheitlichung von Experteninterviews ist ein Leitfaden eine hilfreiche Unterstützung [46, S. 43-46, 48, S. 27-34, 58, S. 676-678]. Ein solcher Leitfaden hilft insbesondere bei der Strukturierung eines Themas und zusätzlich bei der Durchführung des Interviews selbst. Dabei kann ein Leitfaden unterschiedlich stark strukturiert sein, was sowohl Einfluss auf die Anzahl und die Art der verwendeten Fragen als auch deren Reihenfolge hat. Der Grad an Strukturierung hängt von mehreren Faktoren ab, bspw. den Anforderungen, die an den Leitfaden gestellt werden, ebenso wie von den Präferenzen der Interviewer. Dabei können klar formulierte Fragen gewollt sein, um im Bedarfsfall darauf zurückzugreifen. Dies kann vom Interviewer aber auch als einengend wahrgenommen werden und beim Gesprächsverlauf hinderlich sein. Mit der Ausarbeitung des Leitfadens wird schon im Vorfeld festgelegt, welche Art von Interview geführt werden soll.

Im Rahmen dieser Arbeit wurden semistrukturierte Interviews durchgeführt, die eine höhere Anzahl offener Fragen verwenden, Rückfragen ermöglichen und mehr Flexibilität erlauben, um sich dem Interviewten anzupassen. Dabei stört es nicht, wenn der Interviewte selbst das Gespräch auf Themen lenkt, die im späteren Verlauf ohnehin angesprochen würden [58, S. 675]. Durch diese Vorgehensweise wird mit höherer Wahrscheinlichkeit das Ziel erreicht, möglichst viele Informationen zu erlangen [48, S. 28]. Auch spontane Nachfragen helfen, vollständige Antworten zu allen Fragen zu bekommen [48, S. 52, 57, S. 567]. Dabei soll das semistrukturierte Leitfadeninterview einen möglichst natürlichen Gesprächsverlauf finden. Ein Leitfaden kann als Manuskript für die Interviewenden dienen, sollten diese während eines lebhaften Gesprächsverlaufs eine Orientierungshilfe benötigen [52, S. 41, 59, S. 430]. Zudem ist bei der Erstellung des Leitfadens die Reihenfolge der gestellten Fragen im Interview zu beachten. Die verschiedenen Themen sollten einer Argumentationslogik folgen, sodass das Interview für den Experten nachvollziehbar bleibt [60, S. 53]. Ein weiteres Ziel neben der Informationsgewinnung ist es, die Interviewten gleichermaßen dazu zu bewegen, zu den gleichen Themen bzw. gleichen Fragen zu antworten, sodass die Antworten bei der Auswertung miteinander verglichen werden können [47, S. 208, 48, S. 27-28]. 
In einem Experteninterview können gleich mehrere Themengebiete oder Aspekte eines Themengebiets angesprochen werden. Wichtig ist, jeweils Hauptfragen und ergänzende Nachfragen zu entwickeln, um bei Bedarf konkreter zu werden. Es sollte klar sein, welche Fragen verpflichtend zu stellen sind, um die Forschungsfrage zu beantworten. Diese sollen erzählungsgenerierende Fragen ${ }^{2}$ sein, welche den Interviewten dazu einladen etwas auszuholen. Dabei bietet es sich an, nach einer erzählungsgenerierenden Frage mit einer Bewertungs- oder Faktenfrage konkretere Informationen und Aussagen abzufragen [48, S. 62-69]. Generell ist zu berücksichtigen, dass es nicht ein perfektes, einheitliches Vorgehen und es auch keinen perfekten Leitfaden gibt [47, S. 159]. Bezüglich der Länge des Leitfadens werden vier bis acht Hauptfragen für ein 30-minütiges Interview empfohlen. Jede Hauptfrage kann von Unterfragen begleitet werden [52, S. 144]. Es kann hilfreich sein im Leitfaden Zusatzfragen mit aufzunehmen, die als erweiterte Befragung im gleichen Kontext dienen, wenn am Ende des Interviews noch Zeit verbleibt [60, S. 53].

In der vorliegenden Arbeit wurden sechs Hauptfragen, zwei Zusatzfragen und je eine Frage für den Einstieg und das Ende des Interviews gewählt. Die erste Hauptfrage soll in Erfahrung zu bringen, inwiefern sich ein Experte mit Regionalstrom bereits auseinandergesetzt hat und wie dessen Einstellung ggü. Regionalstrom ist. Auf die Meinung zum Regionalnachweisregisters (siehe Kapitel 2.4) geht die zweite Hauptfrage ein. Die dritte Hauptfrage adressiert potenzielle Verbesserungsvorschläge der Experten hinsichtlich der regulatorischen Vorgaben zu Regionalstrom. Die Vor- und Nachteile von Regionalstrom für die Vertriebs- und Beschaffungsseite werden durch die Hauptfragen vier und fünf adressiert. Die letzte Hauptfrage widmet sich der Zukunft von Regionalstrom.

Generell ist es ratsam einen Pretest des Leitfadens durchzuführen, um diesen auf Verständlichkeit, Eindeutigkeit und Vollständigkeit der Fragen sowie die Befragungsdauer zu testen [48, S. 34, 58, S. 682]. Bei großen Anpassungen sollte der Pretest wiederholt werden [46, S. 99]. Aufgrund der großen Bereitschaft von Experten, an einem Interview teilzunehmen (siehe Kapitel 3.5), wurde direkt ein Interview geführt mit der Option, dieses bei (größeren) Anpassungen des Leitfadens zu verwerfen. Dieses Interview ergab keinen Änderungsbedarf am Leitfaden, sodass es ebenfalls in die Auswertung miteinbezogen werden konnte. Der finale Leitfaden ist in Anhang A zu finden.

\subsection{Expertenauswahl und Kontaktaufnahme}

Die Expertenauswahl erfolgte, in Anlehnung an die in [61, S. 196-197] und [62, S. 39] aufgeführten Fragen, anhand der beiden Kriterien (i) Relevanz des Experten zur Beantwortung der Forschungsfrage und (ii) Bereitschaft zur Interviewteilnahme. Die Relevanz eines potenziellen Interviewpartners für die Fragestellung dieser Arbeit bemisst sich anhand des Unternehmens, in dem er tätig ist. Dabei ist zu berücksichtigen, ob das Unternehmen bereits Regionalstrom anbietet, mit Regionalität wirbt, in der jeweiligen Region verwurzelt ist oder das Unternehmen eine relevante Marktstellung ${ }^{3}$ besitzt. Für die Teilnahme eines relevanten Experten an einem Interview ist jedoch auch dessen Bereitschaft vonnöten. Da eine direkte Kontaktaufnahme meist nicht möglich ist, erfolgt diese meist über Dritte, welche die Anfrage an den Experten weiterleiten [48, S. 38]. ${ }^{4}$ Dabei ist es essenziell, den Zweck des Interviews und die positiven Aspekte, u.a. für den Experten selbst, hervorzuheben [62, S. 39].

\footnotetext{
${ }^{2}$ Bogner et al. [48] unterscheiden zwischen fünf verschiedenen Fragetypen, die im Rahmen von Experteninterviews eingesetzt werden können.

${ }^{3}$ Die Einordnung, ob ein EVU eine relevante Marktstellung besitzt oder nicht, erfolgt anhand qualitativer und quantitativer Kriterien. Zu diesen zählen u.a. die Bekanntheit des EVU, bspw. hinsichtlich bestimmter Stromprodukte, aber auch die Stromabsatzmengen oder die mediale Präsenz.

${ }^{4}$ Zu berücksichtigende Punkte bei der Kontaktaufnahme über Dritte sind in [48, S. 37-39] beschrieben.
} 
Die Kontaktaufnahme in der vorliegenden Arbeit erfolgte über ein dreistufiges Verfahren: In der ersten Stufe wurde an potentielle Unternehmen ein Anschreiben per E-Mail verschickt. In diesem Anschreiben wurden das Forschungsvorhaben und seine wissenschaftliche Bedeutung erläutert. Das Anschreiben ist in Anhang B zu finden. Erfolgte innerhalb von ein bis zwei Wochen nach Versand der E-Mail keine Rückmeldung, wurde telefonisch nachgefragt, ob die E-Mail im Unternehmen angekommen sei und diese an einen Experten weitergeleitet wurde. Sofern gewünscht, folgte ein weiteres Anschreiben direkt an einen Experten des jeweiligen Unternehmens. Bei Unternehmen, bei denen dieses Verfahren erfolglos blieb, die aber als relevant für das Forschungsvorhaben eingestuft waren, wurde ein weiteres und letztes Anschreiben über den postalischen Weg versandt, um der Anfrage noch mehr Förmlichkeit und damit Bedeutung zu verleihen.

Hinsichtlich der Stichprobengröße und -zusammensetzung gibt es bei der qualitativen Forschung gegenüber der quantitativen Forschung keine allgemeingültigen Vorgaben [62, S. 39]. Es steht nicht die Repräsentativität der Stichprobe ggü. der Grundgesamtheit im Vordergrund, sondern die inhaltliche Repräsentation [46, S. 39, 48, S. 34-35]. Bei der Stichprobenbildung wurde das sog. theoretische Sampling angewandt, welches die Stichprobe auf Basis bereits erlangter Erkenntnisse sukzessiv erweitert, bis keine weiteren Erkenntnisse mehr gewonnen werden können [46, S. 39, 48, S. 35-36]. ${ }^{5}$ Dabei sind sowohl die Auswahl als auch die Anzahl der Experten primär durch den Untersuchungsgegenstand geleitet [62, S. 40].

Die hohe Bereitschaft seitens Energieversorgungsunternehmen an einem Experteninterview teilzunehmen mündete in 17 durchgeführten Experteninterviews (siehe Kapitel 4). Als Grund hierfür wurde von den Experten insbesondere das Interesse an den Forschungsergebnissen genannt, vor allem Einblicke in das Marktgeschehen sowie in die Sichtweisen anderer EVU. Doch auch altruistische Motive können eine Rolle gespielt haben [48, S. 88]. Des Weiteren kann vermutet werden, dass einige Experten Interviews positiv wahrnehmen, bspw. aufgrund der Möglichkeit, in deren Rahmen die eigene Position zu einem gewissen Thema mitzuteilen [48, S. 88, 60, S. 64].

\subsection{Auswertung}

In Vorbereitung auf die Auswertung von Interviews sind diese im ersten Schritt zu transkribieren und anonymisieren. Dabei sollte der Detailgrad der Transkribtion der Forschungsfrage entsprechend gewählt werden [48, S. 42, 62, S. 53]. In dieser Arbeit wird die wörtliche Transkription mit Übertragung ins Schriftdeutsche verwendet, im Rahmen derer Texte um Dialekte bereinigt und Satzbaufehler verbessert werden [63, S. 89]. Nonverbale Aspekte sind für die Forschungsfrage von untergeordneter Bedeutung. Als Software wird f4transkript verwendet [64]. Durch eine Anonymisierung werden Rückschlüsse auf einzelne Interviewpartner oder zugehörige Unternehmen ausgeschlossen. Dies ist zumeist die Grundlage dafür, dass Experten frei reden und keine Informationen vorenthalten (siehe Kapitel 3.4).

Die Auswertung der Interviews erfolgt mittels der qualitativen Inhaltsanalyse, die für die Informationsgewinnung aus semistrukturierten Leitfadeninterviews als die geeignetste Methode gilt [48, S. 72]. Die qualitative Inhaltsanalyse zeichnet sich durch ein regelgeleitetes Vorgehen aus, bei dem das zu bearbeitende Material identisch behandelt wird [52, S. 204]. Die Inhaltsanalyse der mittels Leitfadeninterviews erhaltenen Daten stellt kein Standardinstrument dar, sondern muss an das Material und den konkreten Forschungs-

\footnotetext{
${ }^{5}$ Eine Vorab-Festlegung der Stichprobenstruktur [46, S. 39, 48, S. 35], bspw. anhand der in Kapitel 4.1 festgelegten Versorgerklassen, lässt sich aufgrund des schwierigen Zugangs zu Experten größerer EVU nur schwer erreichen. Weiterhin ist unklar, ob die Anzahl an EVU einer bestimmten Klasse ein geeigneter Indikator für die inhaltliche Repräsentativität ist. So könnte bspw. auch die Anzahl an Kunden oder die an Kunden gelieferte Strommenge eines EVU als Indikator verwendet werden.
} 
gegenstand angepasst werden [65, S. 51]. Es existieren verschiedene Varianten der qualitativen Inhaltsanalyse [60, S. 90]. Eines der bekanntesten Verfahren ist das Vorgehen nach Mayring [65]. In dieser Arbeit wird jedoch das Verfahren nach Gläser und Laudel [52] angewendet, das auf dem Verfahren nach Mayring [65] aufbaut [52, S. 199]. Es bietet jedoch den Vorteil, dass der Kategorienkatalog induktiv gebildet wird, d.h. dass dieser mithilfe des Leitfadens und der Antworten aus den Interviews entwickelt und sukzessiv angepasst wird, sofern es das Datenmaterial verlangt [52, S. 201]. Diese zusätzliche Flexibilität der Kategorienbildung stellt sicher, dass mehr Informationen aus dem Datenmaterial berücksichtig werden können [52, S. 201]. Die qualitative Inhaltsanalyse nutzt Daten unabhängig davon, wo sie im Text positioniert sind [52, S. 204]. Deshalb ist die Reihenfolge der Fragen während des Interviews von geringerer Bedeutung (siehe Kapitel 3.4). Die Katergorienbildung dient dazu, das transkribierte und anonymisierte Datenmaterial zu reduzieren, indem für die Forschungsfrage relevante Passagen Kategorien zugeordnet werden, um diese im Anschluss zwischen den Interviews zu vergleichen. Ziel ist es, wesentliche Aussagen aus den Interviews zu extrahieren. Als Software zur Auswertung wird f4analyse genutzt [66].

Die Analyse und Interpretation stellen die letzten Schritte bei der qualitativen Inhaltsanalyse dar. Im Rahmen der Analyse wird geprüft, ob die Aussagen der Experten redundant oder widersprüchlich sind [52, S. 202]. Es werden die Aussagen und Meinungen der Experten unter Beibehaltung zentraler persönlicher Zitate zusammengefasst wiedergegeben. Die Zusammenfassungen vermitteln dem Leser die Bedeutung verschiedener Aussagen. Die Nutzung von Zitaten geben dem Leser zusätzlich ein Gefühl für die Art des empirischen Datenmaterials und ermöglichen eine bessere Nachvollziehbarkeit. Außerdem helfen sie den Lesefluss zu verbessern und machen das Zusammengefasste für den Leser greifbarer [52, S. 273-274].

Der letzte Schritt besteht in einer inhaltlichen Interpretation der Analyseergebnisse. Es gilt zu beachten, dass auch bei den vorangegangenen Schritten, d.h. bei der Bildung des Kategorienkatalogs, der Zuordnung von Textstellen zu den Kategorien und der darauf aufbauenden Analyse, ein gewisser Deutungsraum gegeben ist [52, S. 201-202]. Die Deutung erfolgt in Abhängigkeit des Kontexts und der Art, wie Aussagen getätigt wurden. Abbildung 7 stellt die Auswertung grafisch dar.

\section{Interpretation}

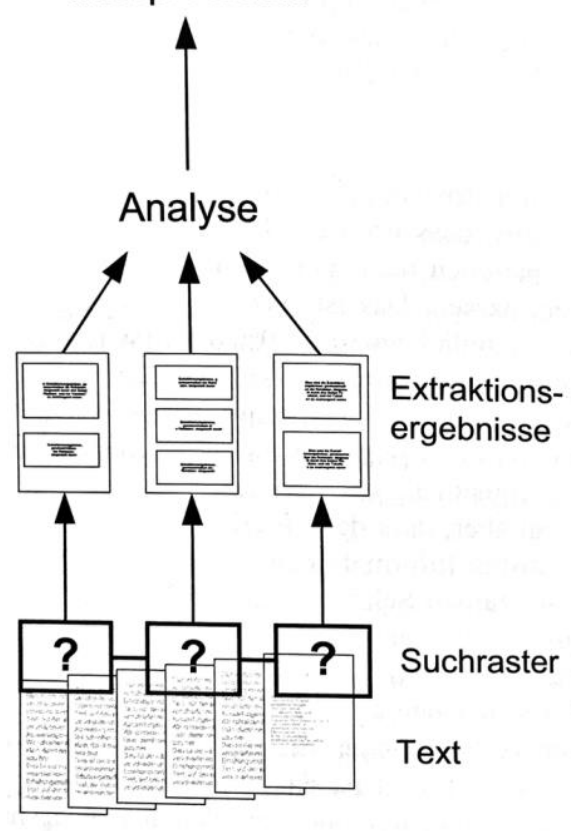

Abbildung 7: Verfahren der qualitativen Inhaltsanalyse (Quelle: Gläser und Laudel [52]) 


\section{Interviews}

Dieses Kapitel gibt einen Überblick über die geführten Interviews. Dabei geht es auf die Versorgerklassen, die Interviewdauern, die Interviewformen und geographische Verteilung der Experten ein.

\subsection{Versorgerklassen}

Die Unternehmen der interviewten Experten werden in verschiedene, eigens für diese Arbeit definierte Klassen eingeteilt. Diese unterscheiden sich hauptsächlich in der Größe des Versorgungsgebietes ${ }^{1}$, der regionalen Verwurzelung und der Bürgernähe.

\section{Überregionale Energieversorger}

Überregionale Energieversorger zeichnen sich durch ein überregionales Angebot und über einen großen Kundenstamm aus, der über das ganze Bundesgebiet oder zumindest große Teile davon verteilt ist. Eine gewisse regionale Verwurzelung kann gegeben sein, ist jedoch für das Image des Unternehmens nicht auszeichnend.

\section{Regionale Energieversorger}

Regionale Energieversorger zeichnen sich durch ihre regionale Verwurzelung aus und das Versorgungsgebiet erstreckt sich über die jeweilige Region. Eine Region kann in diesem Zusammenhang auch ein ganzes Bundesland umfassen.

\section{Kommunale Energieversorger}

Ein klassisches Beispiel für kommunale Versorger sind Stadtwerke, da diese in allen Regionen Deutschlands zu finden sind. Kommunale Versorger zeichnen sich durch ihre regionale Verwurzelung aus. Sie fokussieren sich bei ihrem Versorgungsgebiet auf die jeweilige Gemeinde ${ }^{2}$. Zwischen den kommunalen Energieversorgern finden sich große Unterschiede. So gibt es kommunale Energieversorger mit weniger als 5.000 Letztverbrauchern, jedoch auch mit mehreren Hunderttausend. Letztere decken ein deutlich größeres Versorgungsgebiet ab, sodass der Übergang zwischen kommunalen und regionalen Versorgern flieBend sein kann.

\section{Bürgernahe Energieversorger}

Bürgernahe Energieversorger zeichnen sich durch eine hohe Bürgernähe aus. Hierbei handelt es sich oft um jüngere Unternehmen, die eine große Motivation haben die Energiewende voran zu treiben und dabei nicht ausschließlich gewinnorientiert handeln. Dies passiert unter idealistischeren Ansätzen als bei etablierten Versorgern, die meist Grünstrom- oder Regionalstromprodukte nur zur Ergänzung ihres Produktportfolios anbieten. Die bürgernahen Energieversorger vermarkten meist aus Bürgerenergieanlagen stammenden Strom. Das Absatzgebiet dieses Stroms steht häufig in einem regionalen Zusammenhang zur jeweiligen Erzeugungsanlage. Vereinzelt agieren bürgernahe Energieversorger auch überregional.

\footnotetext{
${ }^{1}$ Unter Größe des Versorgungsgebiets ist in diesem Kontext das Hauptversorgungsgebiet zu fassen. Die vertrieblichen Aktivitäten konzentrieren sich auf dieses. Es ist jedoch nicht auszuschließen, dass auch Stromtarife mit Endkunden außerhalb des Hauptversorgungsgebiets abgeschlossen werden.

${ }^{2}$ Unter dem Begriff „,Gemeinde“ sind auch Städte zu verstehen.
} 


\section{2 Überblick zu den Interviews}

In der nachfolgenden Tabelle 1 sind die geführten Interviews aufgeführt, die im Rahmen dieser Arbeit durchgeführt wurden. Die Spalte „Versorgerklasse“ bezeichnet die Art des Unternehmens (siehe Kapitel 4.1), welchem der jeweilige Experte zuzuordnen ist. Weiterhin sind der Tabelle die Dauern und die Form der Interviews (siehe Kapitel 3.2) zu entnehmen.

Tabelle 1: Überblick zu den durchgeführten Interviews

\begin{tabular}{llll}
\hline ID & Versorgerklasse & Interviewdauer & Interviewform \\
\hline Interview 1 & kommunal & $00: 33: 27$ & Telefoninterview \\
\hline Interview 2 & bürgernah & $00: 50: 35$ & Persönliches Interview \\
\hline Interview 3 & kommunal & $00: 38: 50$ & Persönliches Interview \\
\hline Interview 4 & kommunal & $00: 24: 57$ & Persönliches Interview \\
\hline Interview 5 & bürgernah & $00: 32: 09$ & Persönliches Interview \\
\hline Interview 6 & kommunal & $00: 36: 47$ & Persönliches Interview \\
\hline Interview 7 & bürgernah & $00: 32: 24$ & Telefoninterview \\
\hline Interview 8 & regional & $00: 44: 10$ & Persönliches Interview \\
\hline Interview 9 & bürgernah & $00: 39: 40$ & Persönliches Interview \\
\hline Interview 10 & kommunal & $00: 33: 16$ & Telefoninterview \\
\hline Interview 11 & kommunal & $00: 33: 00$ & Telefoninterview \\
\hline Interview 12 & kommunal & $00: 27: 59$ & Persönliches Interview \\
\hline Interview 13 & kommunal & $00: 31: 09$ & Persönliches Interview \\
\hline Interview 14 & überregional & $00: 33: 27$ & Persönliches Interview \\
\hline Interview 15 & bürgernah & $00: 28: 48$ & Telefoninterview \\
\hline Interview 16 & bürgernah & $00: 36: 23$ & Telefoninterview \\
\hline Interview 17 & regional & $00: 47: 27$ & Telefoninterview \\
\hline
\end{tabular}

\subsection{Geografische Verteilung}

Um die Anonymität der Interviewten und ihrer Unternehmen zu wahren, wird kein Zusammenhang zwischen den Interviews und der geographischen Lage der Unternehmen hergestellt. Zudem ist zu beachten, dass einige Unternehmen - dazu gehören vor allem überregionale und bürgernahe Energieversorger - in mehreren Bundesländern Niederlassungen haben. Folglich ist es schwierig, diesen nur ein Bundesland zuzuordnen. Deshalb wird jedem Interviewpartner das Bundesland zugeordnet, in dem er aktuell arbeitet. Tabelle 2 stellt die geografische Verteilung der Experten dar. 
Tabelle 2: Geografische Verteilung der Interviewpartner

\begin{tabular}{ll}
\hline Anzahl & Bundesland \\
\hline 8 & Baden-Württemberg \\
\hline 3 & Bayern \\
\hline 2 & Nordrhein-Westfalen \\
\hline 1 & Niedersachen \\
\hline 1 & Thüringen \\
\hline 1 & Schleswig-Holstein \\
\hline
\end{tabular}




\section{Ergebnisse}

Nachfolgend werden die Ergebnisse aus den 17 Experteninterviews erläutert. Obwohl es sich bei semistrukturierten Leitfadeninterviews um eine qualitative Methodik handelt, wurden zu Beginn der Interviews die quantitativen Informationen, ob das Konzept Regionalstrom im jeweiligen Unternehmen umgesetzt wird (ja/nein), als auch die subjektive Bewertung der Experten des Konzepts Regionalstrom (eher positiv/eher negativ), abgeleitet. Dies ermöglicht es, ein allgemeines „Stimmungsbild“ der Experten zum Interviewthema zu erhalten.

Von insgesamt 17 Experten setzen 12 das Konzept Regionalstrom bereits um. Zwei weitere äußern während der Interviews, dass sie das Konzept innerhalb der nächsten sechs Monate umsetzen werden. Zwölf Interviewpartner bewerten das Konzept Regionalstrom (eher) positiv, fünf (eher) negativ. Es fällt auf, dass mehr Interviewpartner das Konzept Regionalstrom umsetzen bzw. umsetzen werden als es positiv bewerten. Weiterhin handelt es sich nicht um die gleiche Gruppe von Experten, die das Konzept Regionalstrom umsetzen und dieses zugleich positiv bewerten bzw. umgekehrt. Dies wird einerseits durch politische Vorgaben begründet, bspw., dass ein Landkreis kommunale EVU zur Umsetzung bewegt. Oder durch den Druck, gewisse sich im Markt abzeichnende Trends mitzugehen.

Die hier aufgeführten Kategorien sind mithilfe des Leitfadens (siehe Kapitel 3.4) als auch der gegebenen Antworten entwickelt worden und fassen alle relevanten Inhalte des Datenmaterials zusammen. Dabei wird zunächst auf die Strombeschaffung und den Vertrieb von Regionalstrom aus Sicht von Energieversorgungsunternehmen eingegangen, sowohl mit als auch ohne Nutzung des Regionalnachweisregisters. Daran schließt ein Kapitel hinsichtlich der Frage an, ob und inwieweit Regionalstrom eine größere Gewinnmarge gegenüber konventionellem Strom oder Ökostrom ermöglicht. Das Kapitel endet mit einem Blick in die Zukunft von Regionalstrom und Verbesserungsvorschlägen für die Politik seitens der Experten.

\subsection{Strombeschaffung von Regionalstrom}

In diesem Kapitel wird der Einkauf von Regionalstrom (auch: Strombeschaffung) aus Sicht der Experten beleuchtet. Dabei wird sowohl auf die Frage, ob es genügend Erzeugungsanlagen in der Region gibt, als auch auf das Thema Mehr- und Minderkosten eingegangen. Die Aussagen beziehen sich auf Regionalstrom im Allgemeinen und sind nicht auf Regionalstrom unter Nutzung des RNR beschränkt (siehe Kapitel 2.5).

\subsubsection{Anzahl Erzeugungsanlagen in der Region}

Alle Interviewpartner bürgernaher und kommunaler Versorgungsunternehmen sehen hinsichtlich der Frage, ob genügend Anlagen in der jeweiligen Region vorhanden sind, kaum Engpässe. Es seien sowohl genügend Anlagen vorhanden als auch der Zugriff auf diese Anlagen sei sichergestellt, bspw. durch vorangegangene und bestehende Geschäftsbeziehungen oder die Anlagen befänden sich im Eigentum oder in der eigenen Direktvermarktung. 


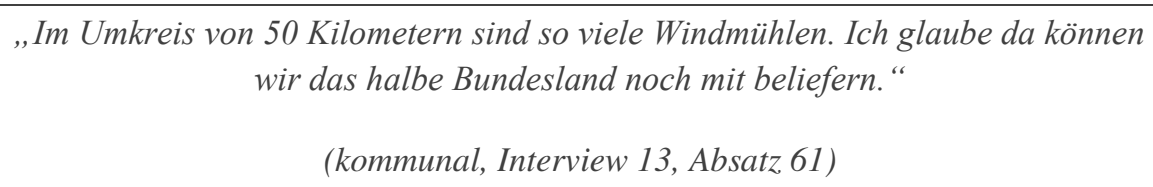

Dabei gilt zu beachten, dass nur sechs der zwölf EVU, die bereits heute das Konzept Regionalstrom umsetzen, das RNR nutzen. Das RNR erweitert den potenziellen Erzeugungspool für ein Regionalstromprodukt um Anlagen, die sich in der EEG-Förderung und dort wiederum in der Veräußerungsform der Marktprämie befinden (siehe Kapitel 2.4). Für die Schaffung eines Regionalstromprodukts kann somit auf mehr Anlagen zurückgegriffen werden. Dafür wird der Regionalitätsbegriff, definiert als $50 \mathrm{~km}$ Umkreis ausgehend vom Postleitzahlengebiet des Letztverbrauchers, relativ eng gefasst. Wird das RNR nicht genutzt, so sind der Definition von Regionalität im Kontext Strom weniger strenge Grenzen gesetzt.

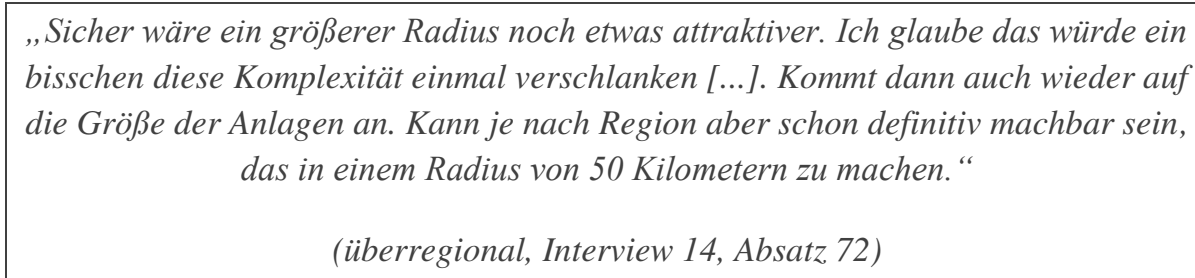

Letzteres mache die Umsetzung des Konzepts Regionalstrom einfacher. Zwei der sechs Interviewpartner, die das RNR nicht nutzen, belieferten in einem ersten Schritt ihre Kunden aus regionalen Anlagen. Reiche deren Erzeugung nicht aus, sprängen weiter entfernte Anlagen ein. Eine Erzeugungskaskade entstehe. Diese Form des Regionalstroms sei jedoch sehr teuer, wenn auf Anlagen zurückgegriffen werde, die sich noch in der EEG Förderung befinden. Allerdings gilt zu beachten, dass regulatorisch mehrere Möglichkeiten bestehen, ein Regionalstromprodukt umzusetzen (siehe Kapitel 2.5).

Die Experten regionaler und überregionaler Versorger berichten auf die Frage, ob genügend Erzeugungsanlagen in den Regionen vorhanden sind, von Fällen, in denen Angebot und Nachfrage nicht zueinander passten.

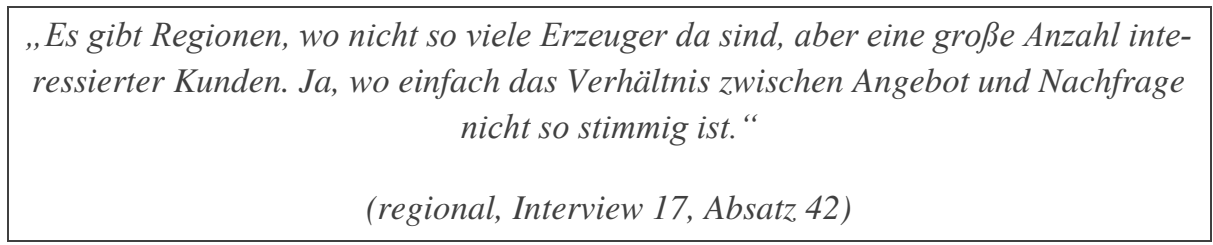

Dies gelte insbesondere bei einer strengen Definition von Regionalität und somit auch bei der Nutzung des RNR. Daher müsse regionen- und unternehmensspezifisch evaluiert werden, ob ein Regionalstromprodukt angeboten werden könne.

\subsubsection{Mehrkosten}

Für Regionalstrom, bei dem das RNR nicht genutzt wird, scheinen nach den Aussagen der Experten die operativen und prozessualen Kosten der Hauptkostentreiber zu sein, z.B. das Managen von mehreren Kleinanlagen im Vergleich zu einer Großanlage sowie die damit einhergehenden Abrechnungsprozesse. Ein Experte eines bürgernahen Versorgers dazu: 


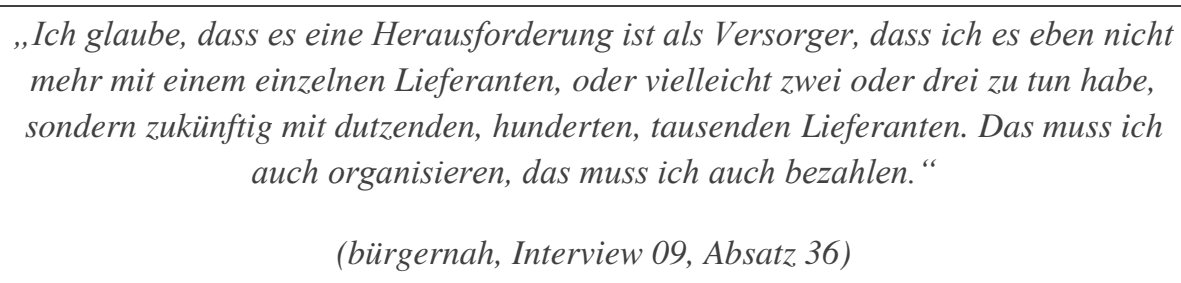

Die Kosten der HKN, wenn sie für die Umsetzung des Konzepts Regionalstrom genutzt werden, seien nach Aussagen der Experten dagegen zu vernachlässigen, wenn diese aus Altanlagen ohne EEG-Förderanspruch stammten. Würden jedoch Neuanlagen mit EEG-Förderansprach verwendet und ,aus dem EEG heraus gekauft“ (siehe Kapitel 2.2), so entstünden hohe Kosten.

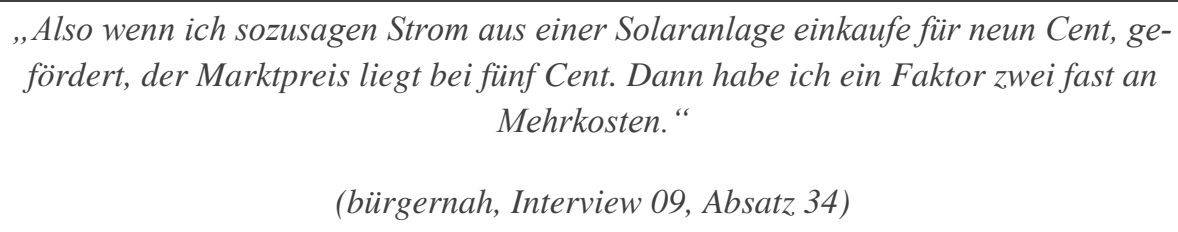

In diesem Fall wechseln die Anlagen in die Veräußerungsform der sonstigen Direktvermarktung ( $21 \mathrm{a}$ EEG), um nicht gegen das Doppelvermarktungsverbot des § 80 EEG zu verstoßen. Daher ist laut Experten auch nur eine Beimischung von Erzeugungsmengen solcher Anlagen wirtschaftlich darstellbar.

\subsection{Strombeschaffung bei Nutzung des Regionalnachweisregisters}

In diesem Kapitel wird darauf eingegangen, ob und inwieweit die neuen Regelungen des RNR einen Einfluss auf die Strombeschaffung haben, insbesondere im Vergleich zu Regionalstrom, bei dem das RNR nicht genutzt wird. Gemäß Leitfaden wurden die Experten darum gebeten, auf die Vor- und Nachteile diesbezüglich einzugehen. Dazu wurden Nachfragen zur Regulatorik sowie im Speziellen zur Kopplung der Regionalnachweise an die vertragliche Lieferkette des Stroms (siehe Kapitel 2.4) gestellt. Bei der Regulatorik wurde separat darauf eingegangen, ob die Experten diese für zu komplex halten.

\subsubsection{Vorteile}

Durch das RNR wird der potenzielle Pool an Erzeugungsanlagen, der für ein Regionalstromprodukt genutzt werden kann, um all diejenigen Anlagen erweitert, die sich in der Veräußerungsform der Marktprämie und somit in der EEG-Förderung befinden (siehe Kapitel 2.4). Obwohl dieser Vorteil in den Interviews nicht explizit hervorgehoben und somit als selbstverständlich von den Experten gesehen wird, ist diese Möglichkeit der Anlagennutzung eine der wesentlichen Neuerungen des RNR. Dieser Vorteil kommt insbesondere dann zum Tragen, wenn die Nachfrage nach Regionalstrom steigt und/oder ansonsten nicht genügend Erzeugungsanlagen in der Region zur Einführung eines Regionalstromprodukts verfügbar sind (siehe Kapitel 2.2). Auf der Beschaffungsseite werden darüber hinaus von den Experten durch das RNR keine weiteren Vorteile gesehen. Einzig ein Experte eines regionalen Versorgers weist darauf hin, dass die Handelsplatzgebühren durch die OTC-Geschäfte, welche die einzig mögliche Transaktionsform bei Nutzung des RNR sind, gegenüber einer Beschaffung über die Börse eingespart werden können. Diese Einsparungen seien jedoch marginal und gelten auch für OTC-Geschäfte ohne Nutzung des RNR. 


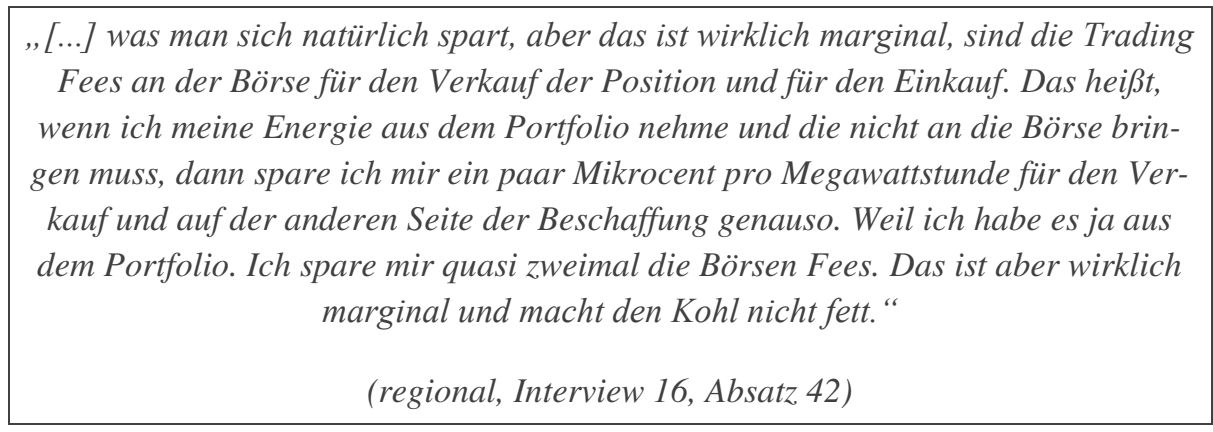

\subsubsection{Nachteile}

Hat ein EVU eigene Erzeugungsanlagen, dann können laut Experten unter anderem die Erzeugungsmengen dieser wenigen, regional differenzierten Anlagen nicht exakt im Voraus abgeschätzt werden. Insofern bestehe ein Risiko darin, dass nicht genügend produziert werde, bspw. durch verschiedene Wetterjahre oder durch Kraftwerksausfälle. Diesen Umstand schildern drei Interviewpartner.

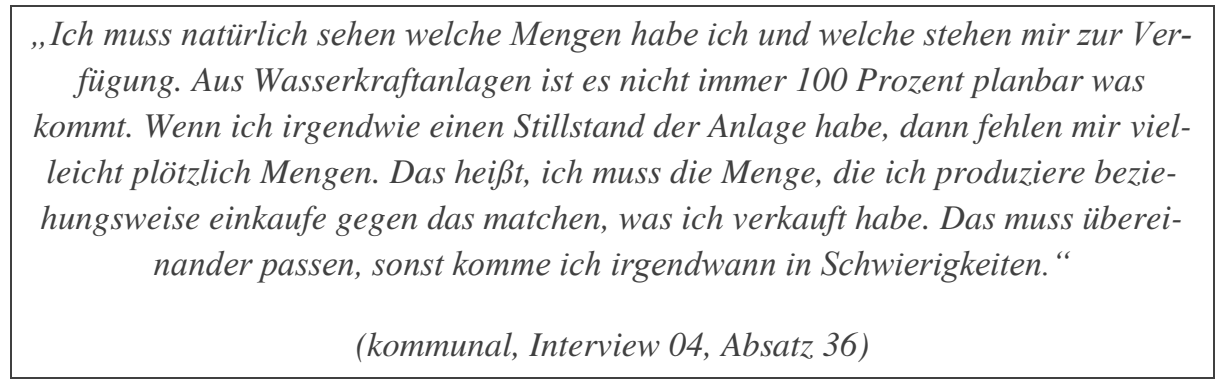

Hat ein EVU keine eigenen Erzeugungsanlagen, so müsse es die Strommengen regional einkaufen. Die regionalen Erzeuger müssten einerseits genügend Mengen produzieren, diese andererseits jedoch auch (langfristig) anbieten, sodass der Vertrieb diesbezüglich Planungssicherheit habe. Darauf wiesen zwei Interviewpartner hin.

$$
\begin{aligned}
& \text { "Nur eben beim Regionalnachweisprodukt bin ich halt irgendwo auf denjenigen, } \\
& \text { der es vor Ort erzeugt, angewiesen." } \\
& \text { (regional, Interview 08, Absatz 64) }
\end{aligned}
$$

Bei Nutzung des Regionalnachweisregisters verringert sich die Marktprämie des Anlagenbetreibers um einen Euro pro MWh ( $\$ 53 \mathrm{~b}$ EEG). Die Kompensation muss daher mindestens einem Euro pro MWh entsprechen, um eine Schlechterstellung des Anlagenbetreibers zu vermeiden, wenngleich in diesem Fall der Mehraufwand, der mit der Nutzung des RNR und einer Vermarktung der RN einhergeht, noch nicht entlohnt wurde. Um einen Anreiz zur Nutzung des RNR auf Erzeuger- bzw. Direktvermarkterseite zu schaffen, muss die Kompensation höher sein.

Diese Kompensations- bzw. Anreizzahlungen durch die Strombeschaffungsseite werden von den drei Interviewpartnern, die das RNR nutzen und keine eigenen Anlagen für ihr Regionalstromprodukt nutzen, jedoch als gering eingestuft. Vielmehr fielen die operativen Mehrkosten von Regionalstrom ins Gewicht, insbesondere, wenn das RNR verwendet werde. 
„Ich glaube die Hauptkosten sind wirklich die ganzen organisatorischen Kosten, die
da anfallen. Sei es die Anmeldung, die Kontenbetreibung, dann die Entwertung und
so weiter, die Verhandlungen mit den Anlagenbetreibern, um letztendlich ja auch
genügend Zertifikate einkaufen zu können."

(kommunal, Interview 12, Absatz 60)

Durch die bilateralen Geschäfte mit den Erzeugern bzw. Direktvermarktern entstünden auf Beschaffungsseite ex-ante Transaktionskosten. Zu diesen zählten Informationsbeschaffungskosten für die Suche nach potenziellen Transaktionspartnern, Anbahnungskosten für die Kontaktaufnahme sowie Vereinbarungskosten. Unter letztere seien Kosten für die Verhandlungen, Vertragsformulierungen und -anpassungen zu fassen. Weitere operative Kosten entstünden durch die individuelle Zuordnung der Kunden zu den Erzeugungsanlagen, da Regionalität ausgehend vom Letztverbraucher definiert wird. Besonders negativ werden durch die Experten der zwei regionalen Versorger und des einen überregionalen Versorgers die nicht vorhandenen IT-Schnittstellen und -Prozesse des RNR hervorgehoben. Dies führe in der Praxis zu händischen Abwicklungen und somit zu Mehrkosten. Dieser Umstand erschwere zudem die Umsetzung des Konzepts Regionalstrom als White Label Lösung ${ }^{1}$. Mehrkosten für die individuelle Zuordnung und den operativen Mehraufwand des Registers entstünden bei Regionalstrom ohne RNR nicht.

"Also momentan ist sicher ein Hemmschuh der prozessuale Aufwand mit den Nach-
weisen. Das ist sicher ein großer Kostentreiber der nicht zu verachten ist und des-
wegen verfolgen wir es ja momentan auch nicht. Weil wir sagen, wir müssten es ent-
weder mit Manpower oder mit großem IT-Aufwand oder beidem wahrscheinlich,
irgendwie operabel machen das Ganze."
(regional, Interview 17, Absatz 40)

Wird Regionalstrom durch den Vertrieb angeboten, so müsse sichergestellt sein, dass auf der Beschaffungsseite möglichst langfristig Mengen gesichert sind. Ansonsten bestehe die Gefahr, dass nach Einführung des Regionalstromprodukts dieses wieder aus dem Portfolio genommen werden müsse, wenn der regionale Erzeuger nicht mehr liefere. Ein Experte wies jedoch darauf hin, dass bei Erzeugern, die ihren Strom für ein Regionalstromprodukt anbieten, ideelle Gründe eine nicht zu vernachlässigende Rolle spielten, weshalb diese Erzeuger ihren Strom meist langfristig zur Verfügung stellten.

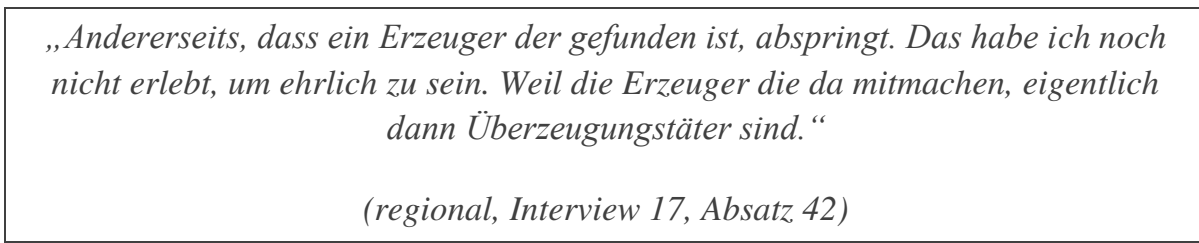

Anlagenbetreiber besäßen vor diesem Hintergrund eine theoretische Marktmacht, wenn sich der Stromvertrieb durch das Anbieten eines Regionalstromprodukts in eine Abhängigkeit begebe. Im aktuellen Marktumfeld sei diese Marktmacht nicht zu beobachten, da die Nachfrage nach Regionalstrom noch gering sei und sich die Versorger gegenüber den Anlagenbetreibern bzw. Direktvermarktern daher in einer besseren Verhandlungsposition befänden. Letztere akzeptierten daher bereits marginale Mehreinnahmen bspw. gegenüber einem Verkauf an der Börse.

\footnotetext{
${ }^{1}$ White Label bezeichnet Güter (Produkte, Dienstleistungen), die von einem Unternehmen hergestellt und durch ein oder mehrere, weitere Unternehmen unter deren Marke vertrieben werden. Anpassungen der ursprünglichen Güter werden vorgenommen, damit diese bspw. mehr den Kundenerwartungen entsprechen oder um mehrere Vertriebskanäle nutzen zu können [67].
} 


$$
\begin{gathered}
\text { "[...] aufgrund der sozusagen besseren Verhandlungspositionen von Vertrieben ge- } \\
\text { genüber Erzeugern." } \\
\text { (bürgernah, Interview 09, Absatz, 10) }
\end{gathered}
$$

Weiterhin seien PPAs² ein mögliches Instrument, Marktmacht zu vermeiden sowie langfristige Geschäftsbeziehungen und damit Sicherheit sowohl für den Erzeuger als auch die Beschaffungsseite zu gewährleisten.

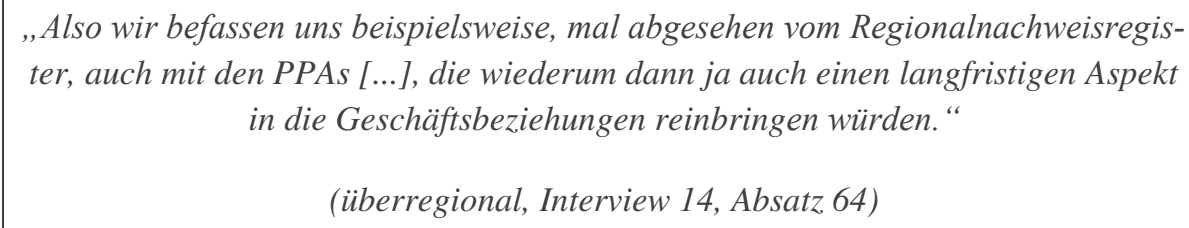

Das Risiko von Anlagenausfällen müsse ebenfalls berücksichtigt werden, bspw. durch redundante Erzeugungsanlagen, welche die gleichen Kriterien erfüllten, insbesondere die Nähe zum Letztverbraucher. Dieses Risiko erwähnen drei Experten verschiedener Versorgerklassen während der Interviews.

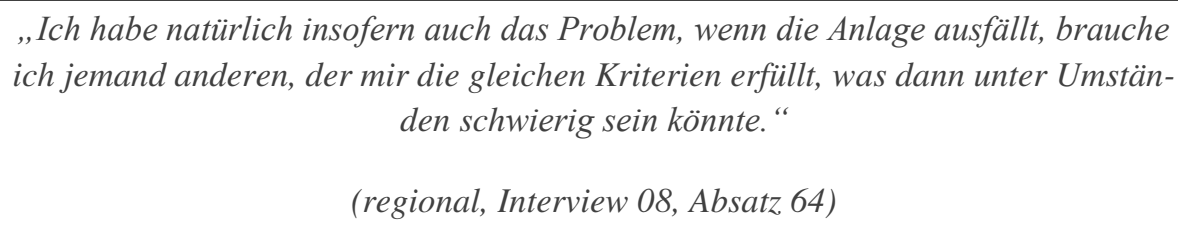

Auch auf der Absatzseite bestehe laut einem Experten die Gefahr, dass sich die Nachfrage nach Regionalstrom über die Zeit verringere. In diesem Fall sei eine langfristige Beschaffung tendenziell negativ, da es für den (in der Regel teurer) beschafften Regionalstrom keinen Abnehmer mehr gebe. Vier Experten regionaler und kommunaler Versorger schildern jedoch, dass Grün- und Regionalstromkunden weniger häufig den Stromtarif wechselten und daher das Nachfragerisiko als gering einzustufen sei.

\begin{tabular}{|} 
„,[...] auch bei den normalen Ökostromprodukten macht man als Vertrieb die Erfah- \\
rung, dass die Wechselquote geringer ist bei diesen Kunden als bei normalen \\
Stromkunden. Meine These bisher: Ja noch geringer dürfte es bei regionalen \\
Ökostromkäufern sein, weil die ja eigentlich ihr Wunschprodukt einmal gefunden \\
haben und somit bei diesem Wunschprodukt auch bleiben wollen. Mit aller Über- \\
zeugung und Gleiches gilt aus meiner Sicht für den Erzeuger." \\
(regional, Interview 17, Absatz 42)
\end{tabular}

\subsubsection{Komplexität für Energieversorgungsunternehmen}

Sieben Experten bemängeln einen zusätzlichen Grad an Komplexität, der mit der Einführung eines Regionalstromprodukts unter Nutzung des RNR einhergehe. Aus den Interviews lässt sich schließen, dass sich diese zusätzliche Komplexität für Energieversorger nicht nur aus den expliziten Anforderungen des Regi-

\footnotetext{
${ }^{2}$ Ein Power-Purchase-Agreement (deutsch: Strombezugsvereinbarung) ist ein langfristiger Stromliefervertrag, der bilateral zwischen einem Käufer (z.B. Letztverbraucher, Lieferant) und einem Verkäufer (z.B. Anlagenbetreiber, Erzeuger) abgeschlossen wird [68, S. 81-82].
} 
onalnachweisregisters ergibt (siehe Kapitel 2.4), sondern auch daraus, dass diese Anforderungen den Interviewten teilweise nicht bekannt sind, bspw. hinsichtlich der Bilanzierungsperiode der Regionalnachweise. Dies kann einerseits durch einen mangelnden Informationsstand der Interviewten begründet sein, jedoch auch aus Unsicherheiten auf Seiten der Experten, wie regulatorisch festgelegte Anforderungen in der energiewirtschaftlichen Praxis umzusetzen sind. Aufgrund dieser Problematik hat die energiewirtschaftliche Branche bereits in der Vergangenheit eigene Leitfäden entwickelt, wie Gesetze zu interpretieren und umzusetzen sind. Ein Beispiel ist der Leitfaden Stromkennzeichnung des BDEW [35].

„Das Thema liegt hier einzig und allein in der nicht vorhandenen Bildungsvor-
schrift, den nicht vorhandenen Rechenvorschriften zum Stromkennzeichnen. Es gibt
da nur einen Leitfaden vom BDEW der dann irgendwann einmal als Branchenstan-
dard letzten Endes herangenommen wird. Aber der BDEW hat keine Richtlinien-
kompetenz."
(kommunal, Interview 01, Absatz 16)

Obwohl nicht direkter Teil des Leitfadens, äußern sich dennoch zwei der sechs Experten, die das RNR bereits nutzen, zur Handhabung des RNR-Portals und befinden diese als in Ordnung.

„,Das Register an sich, der operative Aufwand, das da einzugeben, ist jetzt so von den Kollegen als in Ordnung eingeschätzt worden. "

(regional, Interview 11, Absatz. 12)

\subsubsection{Kopplung der Regionalnachweise an die vertragliche Lieferkette}

Die mit dem RNR einhergehende Kopplung der Regionalnachweise an die vertragliche Lieferkette des Stroms (siehe Kapitel 2.4) mache die Belieferung mit Regionalstrom zwar schwieriger, doch diese Komplexität wird vom überwiegenden Teil der Experten als beherrschbar eingeschätzt. Gewisse Ökostromsiegel setzen eine Kopplung zwischen Stromlieferung und HKN bereits heute voraus, sodass es für die EVU nichts Neues sei. ${ }^{3}$ Zudem wird die Kopplung des RNR von Experten zweier bürgernaher Versorgern sowie einem Experten eines kommunalen Versorgers als energiewirtschaftlich sinnvoll eingestuft.

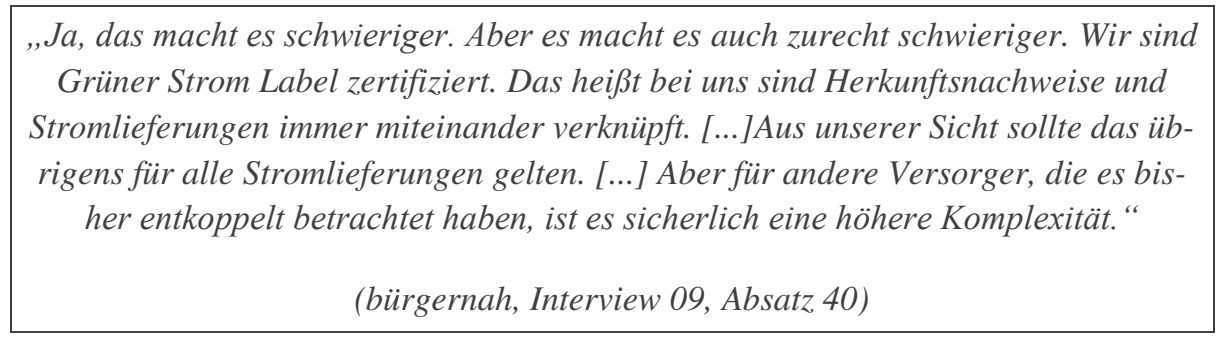

Die Komplexität relativiert sich noch etwas, da zwei Experten kommunaler Versorger davon berichten, die Kopplung des RNR zu umgehen, indem sie nicht regionale Energiemengen aus den eigenen Bilanzkreisen mit regionalen Energiemengen von Direktvermarktern tauschten, wobei die Regionalnachweise bei ihrem Unternehmen verblieben. In Konsequenz werde die Strombeschaffung durch das Regionalstromprodukt nicht tangiert.

\footnotetext{
${ }^{3}$ Ausführungen zur sog. ,,optionalen Kopplung“ des HKNR sind in Kapitel 2.3 zu finden.
} 


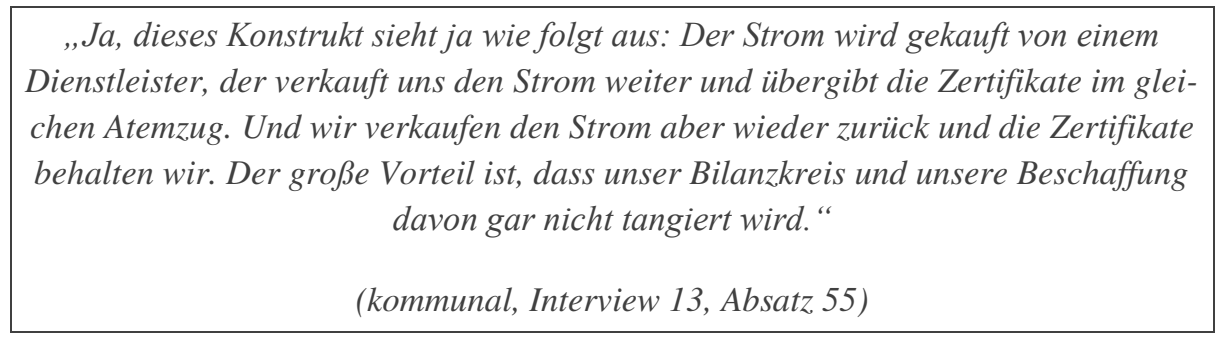

Auf der anderen Seite geht zwei Experten die Kopplung des RNR auf Jahresbasis noch nicht weit genug. Sie fordern für eine bessere Integration der Erneuerbaren in das Energiesystem eine regionale Belieferung in Echtzeit, was jedoch mit gewissen Anreizen vergütet werden sollte (siehe Kapitel 5.8).

\subsection{Vertrieb von Regionalstrom}

Nach Fragen bezüglich der Strombeschaffung widmeten sich die Interviews der vertrieblichen Seite von Regionalstrom. Die Hauptfragen gehen dabei auf die Vor- und Nachteile von Regionalstrom für den Vertrieb ein. Weitere Aspekte, wie der Einfluss von Regionalstrom auf das Image eines Unternehmens und die daraus resultierenden Marketingaspekte, die Zielgruppe für ein Regionalstromprodukt und die Mehrzahlungsbereitschaft der Kunden wurden durch Unterfragen adressiert, sofern die Experten nicht bereits in den Hauptfragen selbst darauf eingingen. Die letzte Unterfrage hat das RNR und dessen spezifische Vor- und Nachteile für den Vertrieb im Fokus. Dies wird getrennt in Kapitel 5.4 dargestellt.

\subsubsection{Allgemeine Vorteile}

Bei Strom handelt es sich um ein sogenanntes Low-Involvement-Produkt [69, S. 6-9]. Entsprechend schwierig ist es, die Aufmerksamkeit und das Interesse des Kunden für diese Produkte zu wecken [70, S. 199]. Die Entscheidungsfindung fokussiert sich deshalb in der Regel auf wenige Differenzierungsmerkmale, wie bspw. den Preis [71, S. 177].

Diesen Aussagen aus der Marketing-Literatur zu Low-Involvement-Produkten stimmen auch die meisten der interviewten Experten zu. Einerseits mangele es an Differenzierungsmerkmalen.

\begin{tabular}{|r|}
\hline „Es ist Strom aus der Steckdose. \\
(regional, Interview 08, Absatz 82) \\
\hline
\end{tabular}

Andererseits bestehe auch nicht das Interesse auf Kundenseite, sich intensiv mit Stromtarifen zu beschäftigen.

„Ja, es ist zu langweilig. Keine Sau interessiert sich für Strom. “

(kommunal, Interview 03, Absatz 63)

Entsprechend schwierig sei es, Involvement zu erzeugen. Dennoch biete nach den Aussagen von vier Experten kommunaler Energieversorger Regionalstrom eine Möglichkeit zur Produkt- und Unternehmensdifferenzierung, insbesondere eine Abkehr der Kunden vom Kaufentscheidungsverhalten, welches sich ausschließlich nach dem Preis richte. 
„Ja, dass man sich vom Wettbewerb differenziert und von dieser Discounter-Mentalität. “

(kommunal, Interview 10, Absatz 7)

Hierdurch könne das Image eines Unternehmens erhöht und beim Produkt Strom ein gewisses Involvement erzeugt werden (siehe Kapitel 5.3.2). Acht Experten aller Versorgerklassen nennen in diesem Zusammenhang besonders häufig die Aspekte Nahbarkeit und Bezug zur Heimat, die dem Produkt eine emotionale Komponente verliehen.

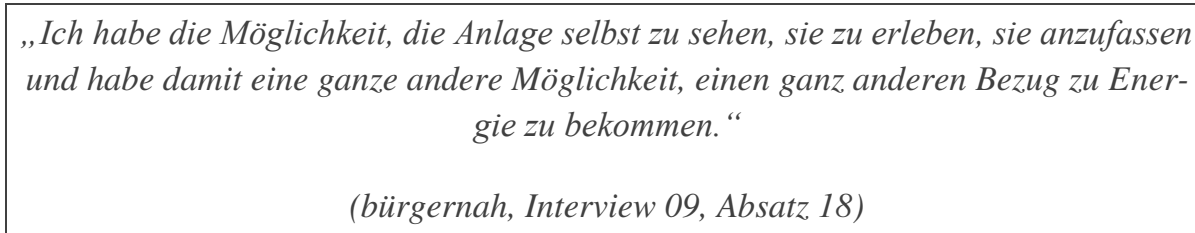

Von vier Experten bürgernaher und regionaler Versorger werden langfristige Kundenbindungen als positives Ergebnis eines höheren Involvements genannt (siehe Kapitel 5.3.4).

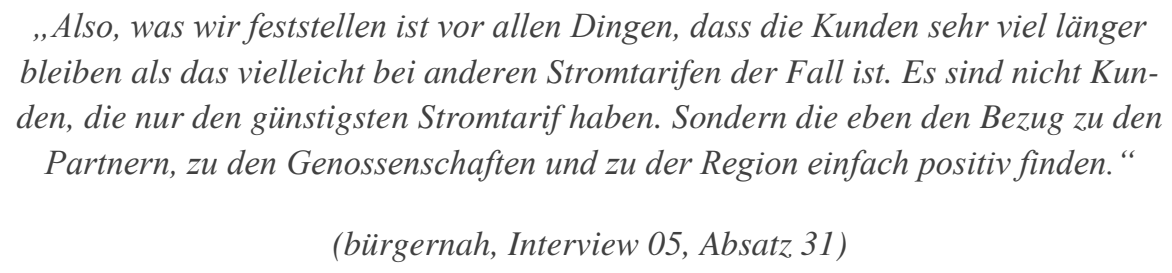

Langfristige Kundenbindungen sind für Energieversorgungsunternehmen besonders vorteilhaft, da die Wechselkosten [72, S. 265] leicht die geringen Margen bei Tarifkunden [73, S. 414] aufzehren können.

\subsubsection{Akzeptanz für Anlagen und Image des Versorgers}

Es existieren zahlreiche Studien, die sich mit der lokalen ${ }^{4}$ Akzeptanz für Erneuerbare-Energien-Anlagen beschäftigen, bspw. [74-78]. Ein Ziel des Gesetzgebers war es unter anderem, mit der Einführung des RNR Anfang 2019 die Akzeptanz von Neuanlagen zu erhöhen, indem Strom konkreter Anlagen vor Ort vermarktet und somit die Akzeptanz durch die lokale Bevölkerung erhöht werde [43, S. 66]. Auch Anlagenbetreiber erhoffen sich durch die regionale Vermarktung ihres Stroms unter anderem Akzeptanz für ihre Erzeugungsanlagen [17, S. 87].

Drei Experten gehen darauf ein, dass Regionalstrom einen ersten Schritt in Richtung Akzeptanz für Erzeugungsanlagen unternehme. Ein Grund dafür sei die Nahbarkeit, welche durch Regionalstrom erhöht werde (siehe Kapitel 5.3.1).

\footnotetext{
${ }^{4}$ Für den Begriff „lokal“ existiert, ebenso wie für die Region (siehe Kapitel 2.1), keine allgemeingültige Definition. In der Regel wird hierunter ein geografischer Raum verstanden, der kleiner ist als die Region [15, S. 23-24].
} 


$$
\begin{gathered}
\text { "[...] regionale Identifizierung mit dem Kunden logischerweise stärken kann und } \\
\text { dadurch natürlich auch die Akzeptanz für diese Anlagen letztendlich erhöhen } \\
\text { kann." } \\
\text { (kommunal, Interview 01, Absatz 16) }
\end{gathered}
$$

Die Akzeptanz sei für Bestandsanlagen allgemein höher als für Neuanlagen. Darauf wiesen drei Experten hin. Daher sei Strom aus diesen Anlagen besonders für ein Regionalstromprodukt geeignet. Somit könnten Bestandsanlagen bei Bedarf ertüchtigt und weiter betrieben werden, z.B. nach dem Auslaufen der EEG-Förderung (siehe Kapitel 5.6.3). Trotzdem solle die Anlagenauswahl für ein Regionalstromprodukt laut drei Experten wohlbedacht durchgeführt und vorab potenzielle Akzeptanzprobleme berücksichtigt werden.

„,Wenn man dann natürlich jetzt, sag ich mal, kritische Anlagen hätte wie große
Freiflächenanlagen auf Ackerflächen, konventionelle Landwirte, die Biogas, betrei-
ben, etc. Dann hat man natürlich auch eher wieder ein Thema das zu Diskussionen
führt. Dann versuchen wir einfach so weit es geht auch unsere Erzeuger dement-
sprechend auszuwählen, dass wir sagen: Das ist eine Energieerzeugung hinter der
wir stehen können. Also keine Windkraft in nicht vernünftigen Gebieten [...]."
(bürgernah, Interview 15, Absatz 36)

Sehr kritisch wird von ebenfalls drei Interviewpartnern der Neubau von Anlagen durch das Produkt Regionalstrom gesehen. Dieser könne auf Ablehnung seitens der Bevölkerung stoßen. Dies gelte insbesondere für Windkraftanlagen, welche die größten Akzeptanzprobleme hätten. Diesen Umstand sprechen sechs Interviewpartner an.

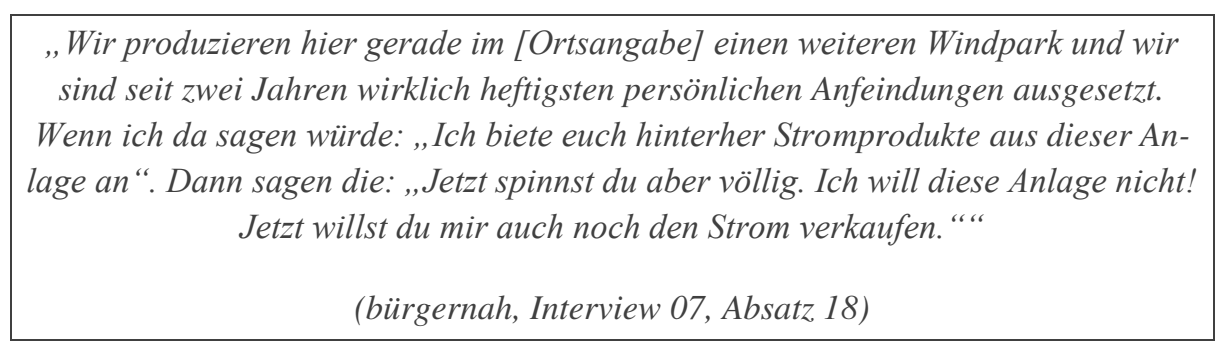

Auf der anderen Seite zeigten die Erfahrungen eines Interviewpartners mit einer Biogasanlage, dass wenn Anlagen erst einmal gebaut seien, die Akzeptanz für diese stark zunehme, da u.a. die anfänglich befürchteten Belästigungen (Lärm, Geruch, Sichtfeldeinschränkungen, etc.) als nicht so schlimm wahrgenommen würden wie befürchtet.

$$
\begin{gathered}
\text { "[...] bis hin sogar, dass durch die Biogasanlage die Geruchsentwicklung deutlich } \\
\text { reduziert ist. Sprich: Da findet sogar eher Akzeptanz statt." } \\
\text { (regional, Interview 08, Absatz 50) }
\end{gathered}
$$

Somit ist es schwierig aus den Interviews allgemeingültige Schlussfolgerungen hinsichtlich der Förderung der lokalen Akzeptanz durch Regionalstrom abzuleiten. Dies bestätigt auch ein Experte eines kommunalen Versorgers der darauf hinweist, dass die Akzeptanz der Energiewende und für die Anlagen vor Ort von Region zu Region sehr unterschiedlich sei. Diese hänge von zahlreichen Aspekten ab, bspw. der Kultur, der regionalen Verwurzelung der Bevölkerung, der Anzahl bereits existierenden Anlagen oder der Stimmung zur Energiewende vor Ort. 
„,...] da wird auch jede Kommune ihren eigenen Weg gehen und auch die Infrastruktur ist sehr unterschiedlich in Deutschland. “

(kommunal, Interview 03, Absatz 67)

Die Akzeptanz könne auf verschiedene Weise gefördert werden: Sei es durch Beteiligungsmöglichkeiten, bspw. in Form von Community-Konzepten ...

„Andererseits, wenn ich davon überzeugt bin, dass es dem Kunden was bringt, dass es der Region etwas bringt, dass so eine Art Community entstehen kann. Ja auch dieser Community-Aspekt ist ja dabei nicht zu verachten, der um sich greift. Ja auch in Großstädten erlebe ich das zunehmend mehr. “

(regional, Interview 17, Absatz 20)

... oder einer echten finanziellen Beteiligung an Anlagen.

„Dann werden Sie Quoten sehen, die unglaublich pro Windkraft sind und obwohl wir hier mitten im Epizentrum der Windkraft sitzen [...], weil fast alle beteiligt sind. Bürgerwindparks: Die schaffen Akzeptanz und schaffen und auch genau das Gegenteil von Blockadehaltung und von Gegenwind."

(regional, Interview 16, Absatz 40)

Aber auch durch positive Argumente, dass die Wertschöpfung vor Ort bleibe ...

„Das ist regional und das ist vor Ort. Das sind dann regionale Wertschöpfungskreisläufe, was ja jeden betrifft. Dann bleibt das Geld in der Region und zudem kommt auch die Energie aus der Region und es ist hundertprozentig nachhaltig. "

(regional, Interview 16, Absatz, 26)

... oder die Versorgungssicherheit der Region erhöht werde.

"Also wenn ich weiß, ,, aus dieser Anlage kommt mein Strom “, dann weiß ich natürlich, dass mein Ort vielleicht eine gewisse Versorgungssicherheit hat und nicht darauf angewiesen ist, dass Strom aus dem Kraftwerk in 500 Kilometern Entfernung kommt. Für uns ist das sowieso ein Akzeptanz-Tool, weil wir glauben, dieser direkte Strombezug oder diese Verbindung dahin durchaus positiv bei einem Menschen ankommt."

(bürgernah, Interview 05, Absatz 59)

Von diesen Argumenten werden die regionale Wertschöpfung und die finanzielle Beteiligung als Akzeptanzvehikel mit sieben Experten besonders oft genannt. Bereits heute könnten sich die Bürgerinnen und Bürger teilweise direkt an Erneuerbare-Energien-Projekten finanziell beteiligen. Eine andere, für die Zukunft denkbare Art der indirekten finanziellen Beteiligung könne eine Vergünstigung bei regionalem Strombezug darstellen. Dies könnte das von drei Experten genannte Neidargumente gegen Regionalstrom abschwächen. Denn je kleiner die Region werde, desto mehr spielten zwischenmenschliche Aspekte eine Rolle, z.B. Strombezug vom Nachbarn, dem Erlöse nicht gegönnt werden. 
Das Wertschöpfungsargument müsse laut zwei Experten bei Versorgern mit einem Regionenbezug relativiert werden, da manche Kunden bereits davon ausgingen, dass die Wertschöpfung bei diesen Versorgern vor Ort bleibe und sie regional beliefert würden. Weiterhin könne das Thema Versorgungssicherheit in Verbindung mit Regionalstrom von der Bevölkerung auch kritisch wahrgenommen werden, wenn die Region und/oder die Heterogenität der Erzeugungsanlagen immer kleiner werde. Hier könnten bspw. Fragen aufkommen, wie die Versorgungssicherheit bei einem regionalen PV-Stromtarif nachts aufrechterhalten werden kann.

Hinsichtlich der Frage, wie sich Regionalstrom auf das Image eines Versorgungsunternehmens auswirkt, sprechen fünf Experten an, dass sie sich durch das Anbieten von Regionalstrom in erster Linie einen Imagegewinn erhoffen.

„Wir haben hier idealerweise einen Imagegewinn. “

(regional, Interview 08, Absatz 34)“

Doch von vier Experten wird auch erwähnt, dass es zu potenziellen Imageverlusten aufgrund mangelnder Akzeptanz für Erzeugungsanlagen kommen könne. Allerdings erfolgten die aktuell beobachteten Widerstände gegen den Ausbau der erneuerbaren Energien durch einen kleinen Anteil der Bevölkerung. Der Widerstand sei jedoch sehr laut und werde daher öffentlich stark wahrgenommen.

„Ich glaube, dass diese Anzahl von Personen marginal gering ist. Die sind bloß sehr laut, das ist richtig. "

(regional, Interview 16, Absatz 40)

Diese Bevölkerungsgruppe würde einen Versorger auch mit Sanktionen belegen (z.B. Kündigungen, Negativwerbung), wenn dieser Veränderungen in ihrem Umfeld durch den Bau neuer Anlagen herbeiführe.

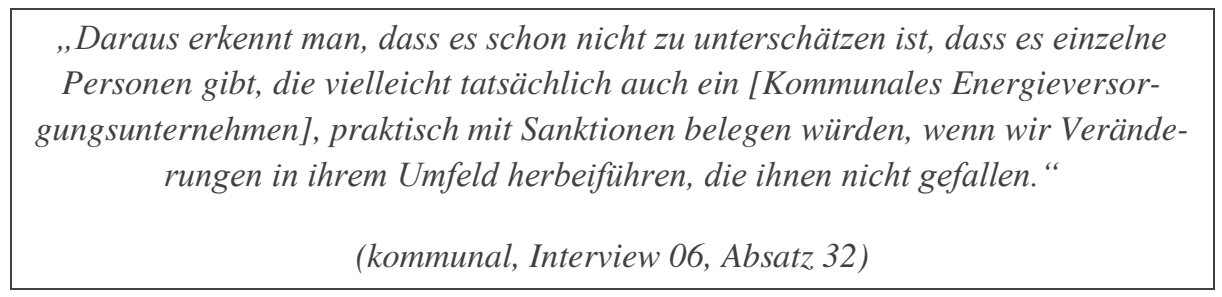

Ein Regionalstromprodukt könne in diesem Fall zumindest in der kurzen Frist die Akzeptanz nicht erhöhen, sondern nur verringern.

\subsubsection{Marketing}

Bei Regionalstrom handelt es sich um ein vergleichsweise neues Konzept in der Energiewirtschaft. Die vier Unternehmen, welche erst vor kurzem ein Regionalstromprodukt in ihr Portfolio mit aufnahmen, haben dieses nach eigenen Aussagen marketingtechnisch bislang nicht oder kaum beworben. Dies könnte eine Erklärung dafür sein, dass eine geringe Nachfrage nach Regionalstrom vorliegt (siehe Kapitel 5.3.5). Insbesondere bei Low-Involvement-Produkten wie Strom wird in der Marketing-Literatur empfohlen, nur wenige Werbebotschaften möglichst oft zu kommunizieren, um auf diese Weise ein passives Lernen zu ermöglichen und ein Bewusstsein für das Produkt beim Kunden zu schaffen [71, S. 177]. Diese Auffassung vertritt auch ein Experte eines kommunalen Versorgers: 
„Also, man muss es auch wissen und nicht nur irgendwo auf der Homepage als Imageträger schreiben nach dem Motto: Wenn einer fragt, kann ich sagen ich habe es. Sondern ich muss es halt auch tatsächlich aktiv bewerben und betreiben. “

(kommunal, Interview 06, Absatz 58)

Erschwert werde das Marketing nach Ansicht von sieben Experten durch den Umstand, dass der Kunde an diesem nur wenig Interesse zeige (siehe Kapitel 5.3.1).

„, Genau, die sind energiemüde. Die wollen das Thema nicht mehr hören. “

(regional, Interview 16, Absatz 24)

Zudem handle es sich bei Regionalstrom um ein erklärungsbedürftiges Produkt. ${ }^{5}$ Darauf machen sieben Experten aller Versorgerklassen aufmerksam.

$$
\begin{gathered}
\text { „Es ist noch zu abstrakt für die meisten. “ } \\
\text { (regional, Interview 08, Absatz 44) }
\end{gathered}
$$

So könne es laut Aussagen von sechs Experten bereits an einer Differenzierung zwischen einem Grünstromtarif und einem regionalen Grünstromtarif scheitern.

$$
\begin{gathered}
\text { „Die Stadtwerke in [Stadt X] sagen „, Wir haben ja schon was und da würden wir ja } \\
\text { nur noch Verwirrung stiften, wenn wir nun noch ein weiteres Produkt aufnehmen “. } \\
\text { Weil, wie gesagt, der Mehrheit der Kunden diese Unterschiede als unverständliche } \\
\text { und zu komplizierte Unterschiede erscheinen. " } \\
\text { (bürgernah, Interview 02, Absatz, 60) }
\end{gathered}
$$

Weiterhin könne es zu Rückfragen und Imageproblemen kommen, da die Kunden bei Ökostromtarifen ggf. bereits davon ausgingen, dass Strom aus Erneuerbare-Energien-Anlagen aus der Region geliefert werde, was jedoch in der Regel nicht der Fall ist.

\begin{tabular}{|} 
„Ja ihr macht das doch alles schon “. Die Kunden in [Stadt X] gehen vielleicht \\
durch die gute Imagewerbung, die unsere Kollegen die letzten Jahre gemacht ha- \\
ben, davon aus, dass sie sowieso Strom aus Erneuerbaren-Anlagen bekommen. Und \\
natürlich sind die auch regional. Was aber nicht stimmt. Und das könnte ein kleiner \\
Bumerang sein." \\
(regional, Interview 11, Absatz, 36)
\end{tabular}

Ein Experte eines bürgernahen Versorgers geht dennoch davon aus, dass es ein gewisses Segment ökoaffiner Kunden gebe, die mittels eines Regionalstromprodukts adressiert werden können. Dabei bestehe jedoch

\footnotetext{
${ }^{5}$ Im Marketing wird ein Produkt als erklärungsbedürftig bezeichnet, wenn es für einen (potenziellen) Kunden nicht oder kaum möglich ist, die Funktion oder den Nutzen des Produkts selbstständig zu erfassen [79].
} 
die Gefahr, dass Kunden, welche aktuell bereits ein Ökostromprodukt beziehen, zum Regionalstromprodukt wechselten. In Summe würden ggf. nicht mehr Kunden erreicht, sondern diese wechselten nur den Tarif. ${ }^{6}$

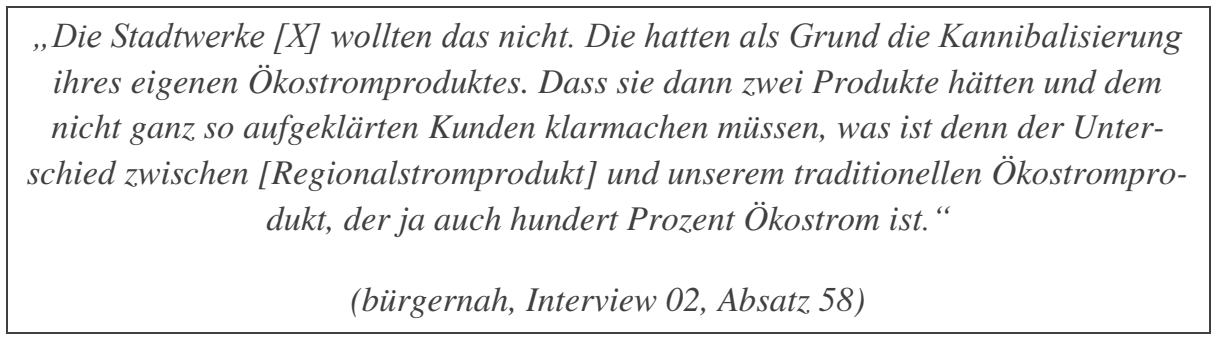

In dieser Argumentation ist allerdings unberücksichtigt, dass nicht nur die Kundengewinnung, sondern auch die Kundenbindung ein mögliches Ziel für die Einführung eines Regionalstromprodukts sein kann (siehe Kapitel 5.3.1).

Von hohen Kosten des Marketings für Regionalstrom seien insbesondere Versorger mit großen Absatzgebieten betroffen, da deren Vertriebsmaterial regionenspezifisch angepasst werden müsse.

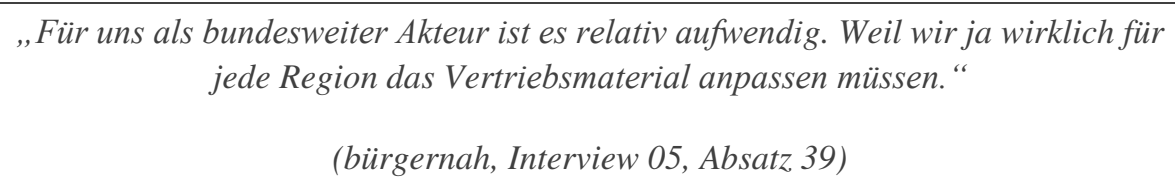

Kommunale und je nach Absatzgebiet auch bürgernahe Energieversorger hätten durch ihre regionale Verwurzelung diesbezüglich einen Vorteil. Für sie entfalle nicht nur die Anpassung des Vertriebsmaterials, sondern sie besäßen auch das Wissen über die Regionen, in denen der Strom vermarket werden soll, z.B. hinsichtlich Kultur, Werten und Einstellungen.

\subsubsection{Zielgruppe}

Fragen bezüglich der Zielgruppen für Regionalstrom sind nicht Bestandteil des Leitfadens. Dennoch äußerten sich mehrere Experten dazu. Diese Informationen sind hier zusammengefasst.

Vier Experten sehen die (potenziellen) Regionalstromkunden als eine Untergruppe der ökoaffinen Kunden. Diese Kunden könnten wiederum unterteilt werden in solche, die aktiv an der Energiewende mitwirken wollen und können, bspw. in Form von Investitionen und Beteiligungen. Die zweite Gruppe bestehe aus Kunden, die die Energiewende lediglich unterstützen wollen oder nicht aktiv mitwirken können. Letzteres könne bspw. durch den Bezug von Regionalstrom oder Ökostrom geschehen. Die Verwendung der Mehrerlöse obliege dann dem jeweiligen Versorgungsunternehmen. ${ }^{7}$

\footnotetext{
${ }^{6}$ Der Absatzrückgang eines Produkts der gleichen Marke, Produktlinie oder des gleichen Unternehmens aufgrund der Einführung eines anderen Produkts wird als Produktkannibalisierung bezeichnet [80, S. 71].

${ }^{7}$ Ein Überblick zu Differenzierungsmöglichkeiten von Ökostromprodukten in Form von Zusatzmerkmalen ist in [1, S. 85-87] zu finden.
} 


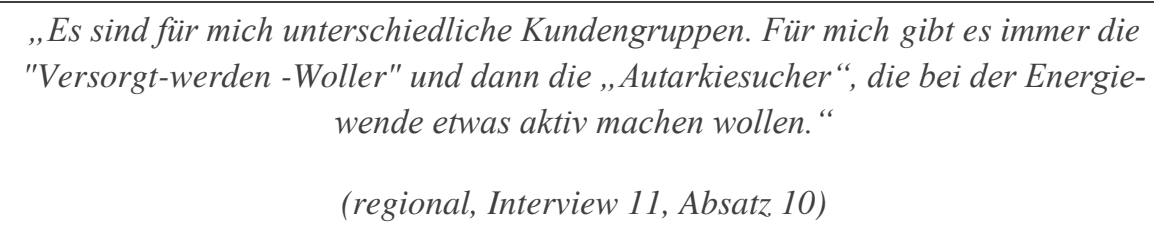

Die Unternehmen von drei Experten, die Aussagen hinsichtlich Zielgruppensegmenten tätigen, konzentrierten sich aktuell auf das Haushaltskundensegment. Jedoch haben in jüngster Vergangenheit vermehrt Gewerbe- und Industriebetriebe ihren Strombezug auf Ökostrom umgestellt [1, S. 91]. Von diesem Trend berichten auch die Experten. Nach deren Ansicht könne dies durch die steigende Wahrnehmung von Umweltschutzthemen in der Öffentlichkeit und die damit indirekten Auswirkungen auf das Unternehmensimage begründet sein. Daher seien Gewerbe- und Industriebetriebe auch für regionalen Grünstrom eine potenzielle und interessante Kundengruppe, da sie größere Abnahmemengen als Haushaltskunden haben. Die Betriebe wiederum könnten in ihrer Außendarstellung mit der Regionalität ihrer Inputgüter werben.

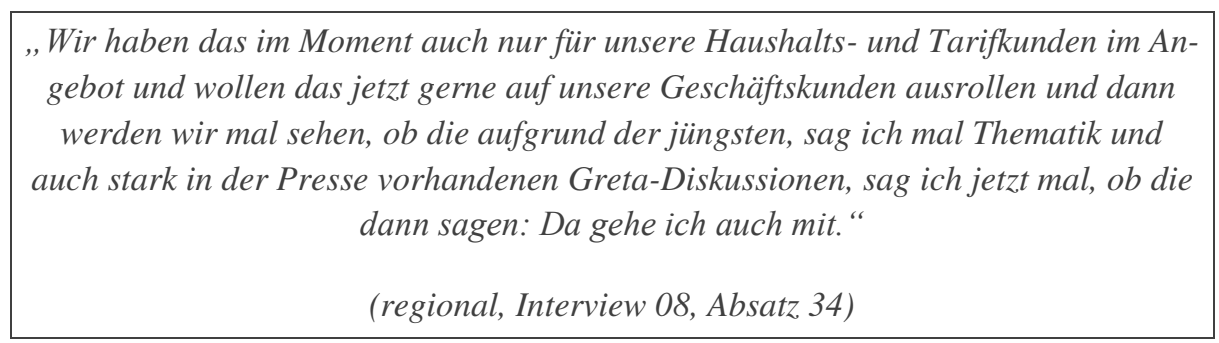

Bei potenziellen Industrie- und Gewerbekunden als Zielgruppe von Regionalstrom könne aufgrund der Bezugsmengen ein kundenspezifisches Marketing und eine individuelle Kommunikation sogar betriebswirtschaftlich sinnvoll sein, was bei Haushaltskunden nicht der Fall sei.

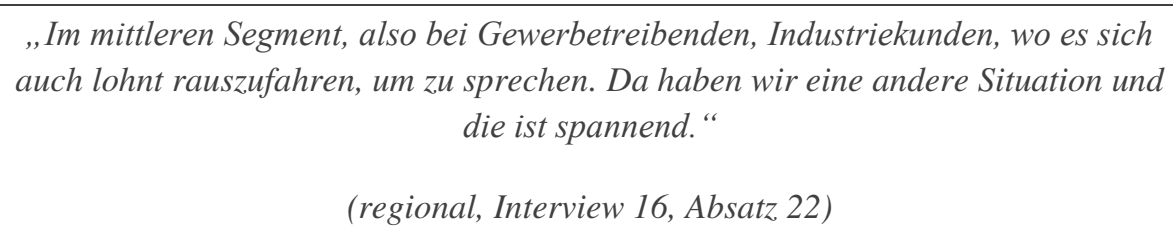

Trotz der von den Experten geschilderten jüngsten Entwicklungen ist allerdings davon auszugehen, dass dieser Wechsel im Gewerbe- und Industriesektor nur stattfindet, wenn sich der Trend hin zu Regionalität im Haushaltskundensegment und auch bei anderen Gütern fortsetzt. In diesem Fall könnte sich der Trend auf den Stromsektor ausdehnen, sodass die Erwartungen der Kunden und damit der Druck auf die Gewerbeund Industriebetriebe steigen.

Hinsichtlich der Unterscheidung zwischen Stadt- und Landbevölkerung erwähnen zwei Experten, dass urbane Räume ein attraktives Vertriebsgebiet darstellten, da auf kleinem Raum eine hohe Anzahl an Kunden beliefert werden könnten und die Stadtbevölkerung zudem stärker auf Trends reagiere, z.B. hinsichtlich Regionalität. 
„Ich könnte mir vorstellen, dass das teilweise passieren wird in Großstädten, wo ich auf sehr kleiner Fläche sehr viele Kunden bedienen kann damit, womöglich. Das könnte ich mir vorstellen. Allerdings ist da auch der Wettbewerb um diese Regionalnachweise relativ hoch. "

(kommunal, Interview 13, Absatz 81)

\subsubsection{Nachfrage}

Die überregionalen, regionalen und kommunalen Energieversorger sehen aktuell im Markt kaum eine Nachfrage nach Regionalstrom, sodass kein Nachfragedruck entstehe.

$$
\begin{gathered}
\text { "[...] der Nachfragedruck fehlt, dass einer kommt: „Ich möchte genau von diesem } \\
\text { Windrad den Strom haben “. [...] Eigentlich kenne ich solche Anfragen nicht." } \\
\text { (kommunal, Interview 03, Absatz 11) }
\end{gathered}
$$

Dies könne durch das mangelnde Bewusstsein der Verbraucher für Regionalstrom bedingt sein (siehe Kapitel 5.3.1). Mithilfe von Marketing könne dieses gestärkt und so ggf. eine Nachfrage erzeugt werden (siehe Kapitel 5.3.3), was jedoch zuerst einmal Kosten für die Versorgungsunternehmen bedeute. Einige der Interviewpartner bürgernaher Versorger sehen jedoch bereits aktuell eine Nachfrage nach Regionalstrom.

„Ja, aus unserer Sicht gibt es ein klares Kundenbedürfnis für Regionalstrom. “

(bürgernah, Interview 09, Absatz 4)

Insofern ergibt sich hinsichtlich der Nachfrage nach Regionalstrom kein eindeutiges Bild. Dennoch lässt sich aus den Interviews eine eher geringe Nachfrage ableiten, da die Aussagen der Experten bürgernaher Versorger, die bei deutschlandweiter Betrachtung eine vergleichsweise geringe Absatzmenge an Strom haben, relativiert werden müssen.

\subsubsection{Mehrzahlungsbereitschaft}

Alle Experten sind sich darin einig, dass es eine Mehrzahlungsbereitschaft einer gewissen Kundengruppe (z.B. ökologische vs. preissensitive Kunden) für Regionalstrom gebe, wobei die relativen Anteile der Kundengruppen von Region zu Region verschieden seien.

$$
\begin{gathered}
\text { „Ja, ich glaube es gibt eine Zusatzzahlungsbereitschaft in einer Teilzielgruppe von } \\
\text { Menschen, die sich das leisten können." } \\
\text { (bürgernah, Interview 09, Absatz, 22) }
\end{gathered}
$$

Da ein Kunde nur schwer zwischen Regionalstrom unter Nutzung des RNR und sonstigem Regionalstrom (siehe Kapitel 2.5) unterscheiden könne, sei laut Experten davon auszugehen, dass sich die Mehrzahlungsbereitschaft zwischen diesen Produkten nicht unterscheide. Es dürfte mehr auf die Kernbotschaften ankommen, welche durch das Marketing vermittelt werden (siehe Kapitel 5.3.3). 


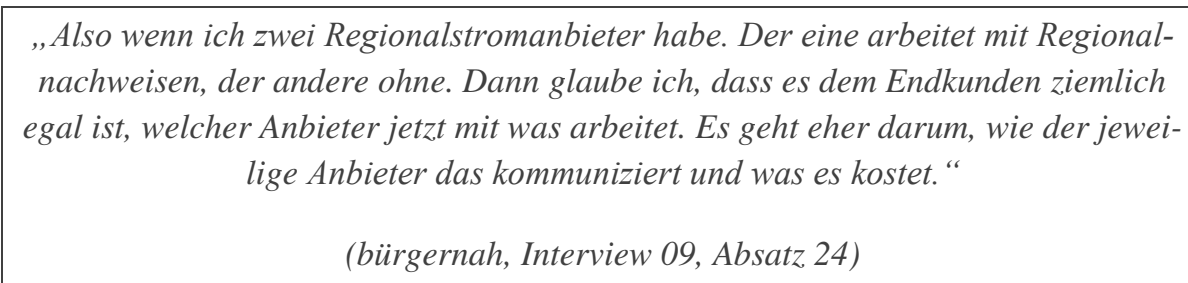

Die absolute Höhe der Mehrzahlungsbereitschaft sei jedoch (stark) begrenzt, da die Kunden im Stromsektor preissensitiv seien. Dies könne durch die im internationalen Vergleich hohen Strompreise in Deutschland begründet sein, sodass die Mehrzahlungsbereitschaft bereits regulatorisch größtenteils ausgeschöpft sei. ${ }^{8}$

$$
\begin{gathered}
\text { „Unser Strom in Deutschland ist teuer. “ } \\
\text { (kommunal, Interview 04, Absatz 56) }
\end{gathered}
$$

Die Mehrzahlungsbereitschaft, obwohl in ihrer absoluten Höhe und auf ein bestimmtes Kundensegment begrenzt, resultiere aus dem guten Gefühl (Fachbegriff: „warm glow of giving“99), sei es für den Ausbau erneuerbarer Energien in der Region, aber auch vereinzelt für soziale Projekte in der Region (z.B. Unterstützung des Fußballvereins), für die ein Teil der Mehreinnahmen eingesetzt werden [1, S. 97]. ${ }^{10}$

$$
\begin{gathered}
\text {,[...] Kunden, die uns 1,5 oder 1,8 Cent spenden pro Kilowattstunde und wir ma- } \\
\text { chen irgendwas Gutes damit im regionalen Umfeld. Sie lehnen sich zurück, die zah- } \\
\text { len gern } 100 \text { Euro mehr und sind zufrieden damit." } \\
\text { (regional, Interview 11, Absatz, 10) }
\end{gathered}
$$

Der höhere Preis für ein Regionalstromprodukt könne jedoch nicht durch Mehraufwand gerechtfertigt werden, z.B. für bürokratische Prozesse oder Nachweise (siehe Kapitel 5.2.2 und 5.3.4).

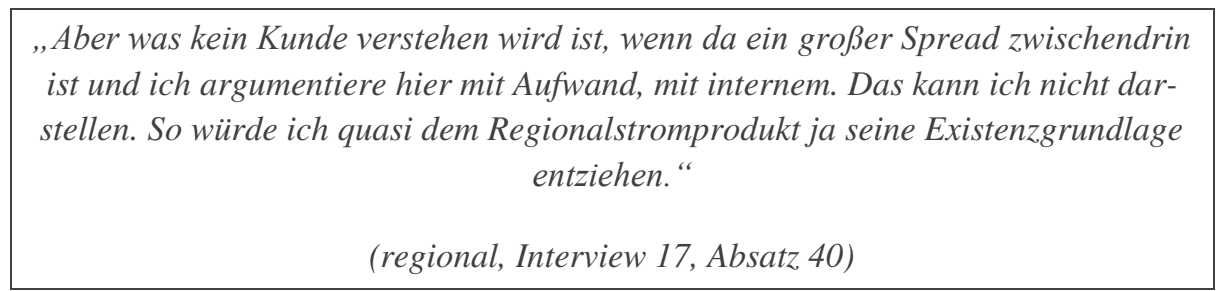

Dieser Umstand ist für Energieversorgungsunternehmen betriebswirtschaftlich ungünstig, da insbesondere bei der Strombeschaffung (siehe Kapitel 5.1 und 5.2) sowie dem Marketing (siehe Kapitel 5.3.3) als Teil des Vertriebs Mehrkosten entstehen.

\footnotetext{
${ }^{8}$ Deutschland hatte 2019 im Ländervergleich der EU-28 die höchsten Strompreise für Haushaltskunden [81].

${ }^{9}$ Unter dem Begriff "Warm-glow of giving" wird der Umstand beschrieben, dass Leute nicht indifferent ggü. ihres eigenen Beitrags zu einem öffentlichen Gut (z.B. Umweltschutz) sind. Die Gründe hierfür können vielfältig sein, bspw. Anerkennung, Erleichterung des schlechten Gewissens, etc. [82, S. 1220].

${ }^{10}$ Ein Überblick zu Differenzierungsmöglichkeiten von Ökostromprodukten in Form von Zusatzmerkmalen ist in [1, S. 85-87] zu finden.
} 


\subsection{Vertrieb bei Nutzung des Regionalnachweisregisters}

In diesem Kapitel sind sowohl Vor- als auch Nachteile zusammengefasst die von den Experten speziell für das RNR genannt wurden und zusätzlich zu den schon zuvor genannten Aspekten zu sehen sind (siehe Kapitel 5.3).

\subsubsection{Vorteile}

Der von allen Interviewten genannte Vorteil des RNR ist die anlagenscharfe Ausweisung der Stromlieferung gegenüber dem Kunden unter dem Rückgriff auf Anlagen in der Veräußerungsform der Marktprämie. Durch diese Ausweisung, bspw. in Form eines Fotos, könne marketingtechnisch eine Story erzählt und somit mehr Involvement erzeugt werden (siehe Kapitel 5.3.1).

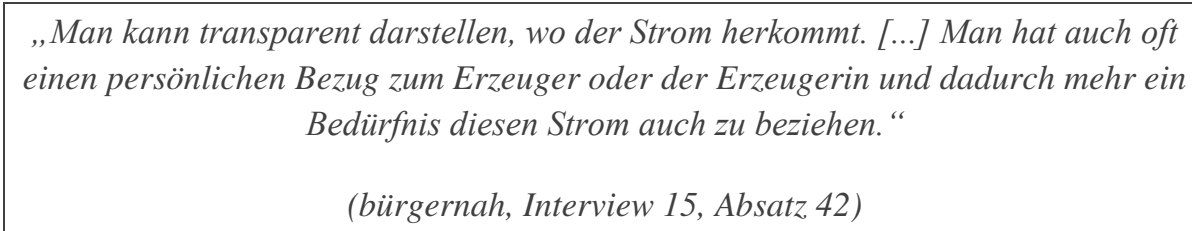

Allerdings ist bisher nicht abschließend geklärt, welche Vermarktungsvarianten regionaler Stromprodukte rechtlich zulässig sind (siehe Kapitel 2.5). Bei einem Interviewpartner wog diese rechtliche Unsicherheit so stark, dass er von einer Nutzung des RNR absah.

\subsubsection{Nachteile}

Ein von vier Experten in den Interviews genannter Nachteil des RNR für den Vertrieb ist der Umstand, dass der Regionalstromanteil auf den EEG-Anteil begrenzt ist (siehe Kapitel 2.4).

„Ich kann kein 100 Prozent Regionalstromprodukt machen. “

(regional, Interview 08, Absatz 56)

Zugleich wird die Bedeutung der Stromkennzeichnung für den Letztverbraucher durch die Experten jedoch als gering eingestuft. Vielmehr erwähnen fast alle Interviewpartner, dass für den überwiegenden Anteil der Letztverbraucher nur die Story zum Produkt Regionalstrom wichtig sei (siehe Kapitel 5.3.6).

Man kann ja einfach sagen: „Schau mal, hier siehst du, da vorne wird dein Strom produziert". Das ganze operative muss den Bürger auch nicht so interessieren. "

(kommunal, Interview 06, Absatz 34)

Kompliziert werde es dagegen, wenn ein Kunde das Regionalstromprodukt vollumfänglich verstehen möchte und in diesem Zusammenhang Nachfragen stelle. So sei gegenüber diesen Kunden nur schwer vermittelbar, wieso in der Stromkennzeichnung lediglich der EEG-Anteil als regional ausgewiesen werden kann oder wieso in mehr als $50 \mathrm{~km}$ Umkreis zur Anlage das jeweilige Regionalstromprodukt nicht mehr verfügbar ist (siehe Kapitel 2.4). 


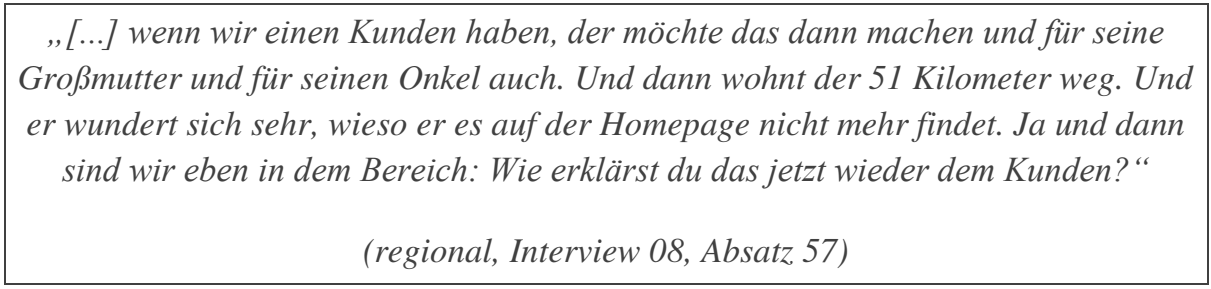

Aus diesen Äußerungen der Experten lässt sich schließen, dass die regulatorische Begrenzung der Regionalität auf den in der Stromkennzeichnung ausgewiesenen EEG-Anteil zu negativen Auswirkungen (z.B. auf das Image) für einen Energieversorger führen kann, wenn es diesem nicht gelingt, dem Kunden das Regionalstromprodukt unter Nutzung des RNR zu erklären.

\subsection{Gewinnmarge von Regionalstrom}

Die (potenzielle) Gewinnmarge bei Regionalstrom ergibt sich aus den (Mehr-)Erlösen abzüglich der (Mehr-)Kosten auf der Beschaffungs- als auch Vertriebsseite. Die Experten wurden daher gebeten, eine Einschätzung der zu realisierenden Gewinnmargen für ein Regionalstromprodukt zu treffen.

Alle Interviewten sind sich einig, dass die Gewinnmarge eines regionalen Ökostromprodukts vergleichbar sei mit der eines klassischen Ökostromprodukts. Die Gewinnmarge sei eventuell sogar geringer, da ein höherer Aufwand für zusätzliche Prozesse, Bürokratie und ein regionenspezifisches Marketing den gleichen oder nur marginal höheren Mehrzahlungsbereitschaften gegenüberstehe (siehe Kapitel 5.1, 5.2 und 5.3.6). Der höhere Aufwand gelte insbesondere bei Nutzung des Regionalnachweisregisters.

„Gewinnmargen sind da durchaus machbar. Aber ich würde jetzt mal sagen, nicht
abweichend von anderen Ökoprodukten. Keine höheren Gewinnmargen, weil Sie
haben ja umgekehrt auch höheren Aufwand. Den dürfen sie ja nicht vergessen."

(regional, Interview 17, Absatz 24)

Es ist zu beachten, dass bei denjenigen Interviewpartnern, die bereits ein neues Regionalstromprodukt unter Nutzung des RNR zusätzlich zu ihren bereits bestehenden Stromprodukten einführten, bislang nur eine sehr geringe Anzahl an Kunden das Regionalstromprodukt beziehen. Es können somit keine Skaleneffekte realisiert werden. Eine andere Situation könnte sich ergeben, wenn großflächig auf das Regionalstromprodukt umgestellt wird. Hier liegen jedoch keine Erfahrungen der Experten vor, ob die Bestandskunden bei einer (starken) Preiserhöhung nicht zu einem anderen Anbieter wechselten, da der einzige Interviewpartner, der diese vollständige Umstellung vollzogen hat, nur marginale Preisanpassungen vornahm.

Neben den monetären Größen sind bei Regionalstrom auch die nicht-monetären Größen zu berücksichtigen, insbesondere der Imagegewinn eines Unternehmens (siehe Kapitel 5.3.2). Unter Abwägung der von den Experten genannten Aspekten wirft der aktuell geringe Nachfragedruck (siehe Kapitel 5.3.5) dennoch die Frage auf, ob Energieversorger, die nach rein finanziellen Gesichtspunkten am Markt agieren, ein Regionalstromprodukt aufsetzen sollten, wenn selbst bei stärkerer Marktpenetration keine oder nur marginal höhere Gewinnmargen resultieren. Alle Interviewpartner, die bislang ein Regionalstromprodukt als zusätzliches Produkt im Portfolio haben, machten dies nach eigenen Aussagen zu Testzwecken oder da es von der Kommunalpolitik gewünscht ist. Ein großes Marktpotenzial ${ }^{11}$ wird nicht gesehen.

\footnotetext{
${ }^{11}$ Das Marktpotenzial bezeichnet die mögliche Absatzmenge eines Produkts bei optimaler Entwicklung des betrachteten Marktes [83, S. 72].
} 


\subsection{Zukunft von Regionalstrom}

Die letzte Hauptfrage des Leitfadens widmet sich der Zukunft von Regionalstrom. Dabei wurden die Experten um eine generelle Einschätzung diesbezüglich gebeten, wobei der überwiegende Anteil der Experten Aussagen zur Absatzentwicklung tätigten. Weiterhin wurde mittels drei Unterfragen auf weitere Teilbereiche eingegangen. Dabei wurden die Experten gefragt, was für einen Einfluss das Ausscheiden der ersten Erzeugungsanlagen aus der EEG-Förderung für Regionalstrom bedeuten wird, welche Versorgerklasse das Konzept Regionalstrom am besten umsetzen kann und ob Regionalstrom helfen wird, die Akzeptanz der Energiewende vor Ort in Deutschland zu erhöhen. Letzteres ist einer der Gründe, weshalb der Gesetzgeber das Regionalnachweisregister eingeführt hat (siehe Kapitel 2.4).

\subsubsection{Absatzentwicklung}

Drei Interviewpartner schildern, dass Regionalität - analog zum Lebensmittelsektor - auch in Zukunft ein relevantes Produktmerkmal sein werde. Jedoch werde Regionalität laut dieser Experten für die Bevölkerung beim fortschreitenden Ausbau der Erneuerbaren ein selbstverständlicheres Produktmerkmal beim Strombezug sein. Das Wachstum weiterer Sektoren, z.B. der Elektromobilität, könne nach Ansicht eines Experten den Absatz nach Regionalstrom beschleunigen.

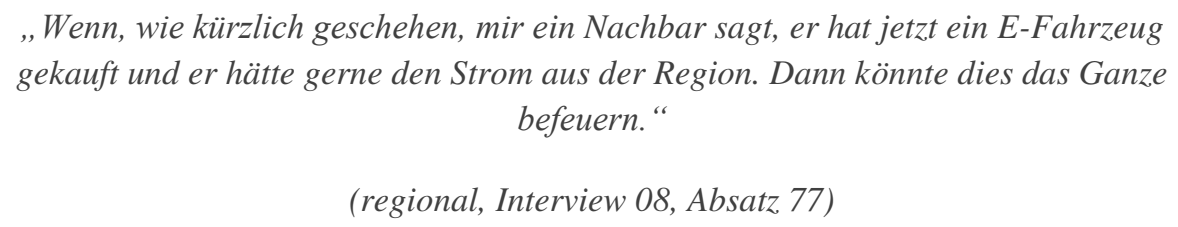

Nahezu alle Interviewten sind sich einig, dass Regionalstrom in seiner jetzigen Form ein Nischenprodukt sei und mit hoher Wahrscheinlichkeit bleiben werde.

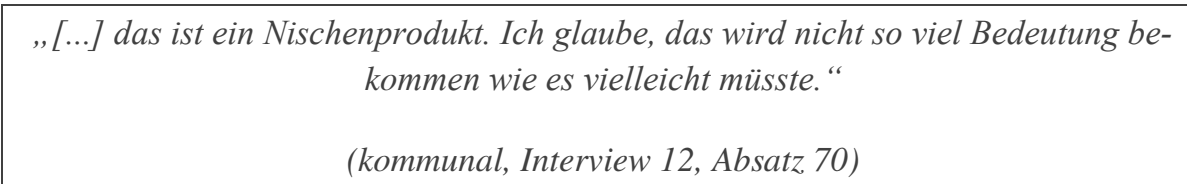

Das Kundensegment ökoaffiner Kunden sei zwar wachsend, jedoch endlich. Da Regionalstromkunden vermutlich eine Subgruppe der ökoaffinen Kunden sind (siehe Kapitel 5.3.4 und 5.3.5), sei das Marktpotenzial stark begrenzt.

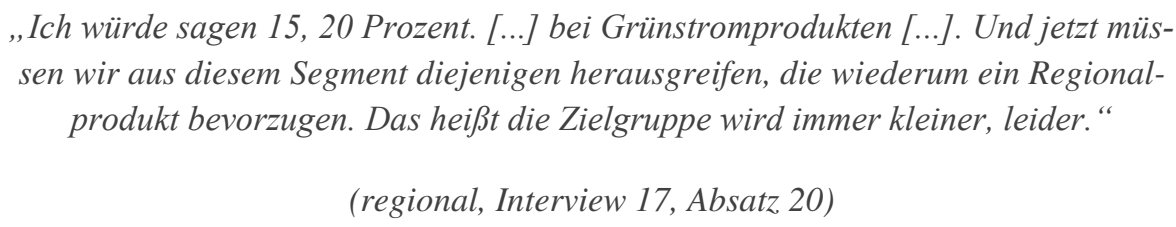

Der Wandel von einem Nischenprodukt zu einem massenfähigen Produkt könne nur vollzogen werden, wenn ökonomische Anreize durch Änderungen in der Regulatorik gesetzt würden, d.h. Regionalstrom Vergünstigungen gegenüber nicht regionalem Strom erhalte, bspw. in Form reduzierter Abgaben, Umlagen und Entgelte. 
„Ich glaube, dass Regionalstrom, solange es teurer ist als herkömmlicher Strom, ein Nischenprodukt bleiben wird. "

(bürgernah, Interview 09, Absatz, 28)

In seiner heutigen Form bezeichnet ein Experte Regionalstrom sogar als marktunfähig.

$$
\begin{gathered}
\text { „In seiner jetzigen Ausgestaltung halte ich es für marktunfähig. Wie gesagt einfach } \\
\text { durch die Situation, dass man von vorne herein schon mal einen Euro extra abfüh- } \\
\text { ren muss. Für den Verwaltungsaufwand des UBA." } \\
\text { (regional, Interview 16, Absatz 38) }
\end{gathered}
$$

Ein Experte eines kommunalen Versorgungsunternehmens schildert als ein mögliches Szenario die Inflation des Wertes von Regionalstrom. Er sieht Parallelen zum heutigen Grünstrommarkt, bei dem die günstigsten Ökostromtarife nur marginal teurer oder gleich teuer wie Stromtarife mit konventionellem Strommix sind, jedoch mit dem Unterschied, dass die wenigen, großen Versorger gegenüber den zahlreichen, kleinen dezentralen Erzeugern ihre Marktstellung ausspielten.

\begin{tabular}{|c|}
\hline "Also bei den jetzigen Regularien - so wie es heute gelebt wird - werden neue \\
Player auf den Markt treten, die aber alle nationalen tätig sind und nicht regional. \\
Also ein Regionalstromprodukt wird von national agierenden oder internationale \\
agierenden Unternehmen geprägt werden. Und dann sind wir im gleichen Modus \\
wie jetzt die Landwirte, die Produzenten sind und über die Discounter gezwninen \\
werden, zu Dumpingpreisen zu verkaufen. Und genauso wird im Energiemarkt pas- \\
sieren." \\
(kommunal, Interview 10, Absatz 61)
\end{tabular}

Die Aussagen der Experten lassen vermuten, dass Regionalstromprodukte in ihrer heutigen Form nur für eine gewisse Kundengruppe interessant und die Absatzsteigerungen stark begrenzt sind. Zudem könnte die Eigenschaft der Regionalität zukünftig kein Produktmerkmal mehr sein, das einer expliziten Erwähnung bedarf, da aufgrund des Ausbaus der erneuerbaren Energien meist ein Bezug zu Erzeugungsanlagen hergestellt ist.

\subsubsection{Produktausgestaltung}

Einzelne Interviewpartner haben sich zu möglichen, alternativen Produktausgestaltungen von Regionalstrom geäußert.

Fünf Interviewpartner können sich die Umsetzung des Konzepts Regionalstrom in Form eines lokalen Energiemarktes vorstellen. ${ }^{12}$ Allerdings müssten in einem solchen Modell die Transaktionen zwischen Erzeugern, Verbrauchern und Prosumern möglichst automatisiert durchgeführt werden, sodass maximal die Handlungsempfehlungen, bspw. eines Gebäudeenergiemanagementsystems, akzeptiert oder abgelehnt werden müssten.

\footnotetext{
${ }^{12}$ In der wissenschaftlichen Literatur hat sich der Begriff „lokale Energiemärkte“ etabliert, wobei - ebenso wie bei der Regionalität der geografische Raum nicht eindeutig definiert ist (siehe Kapitel 2.1).
} 


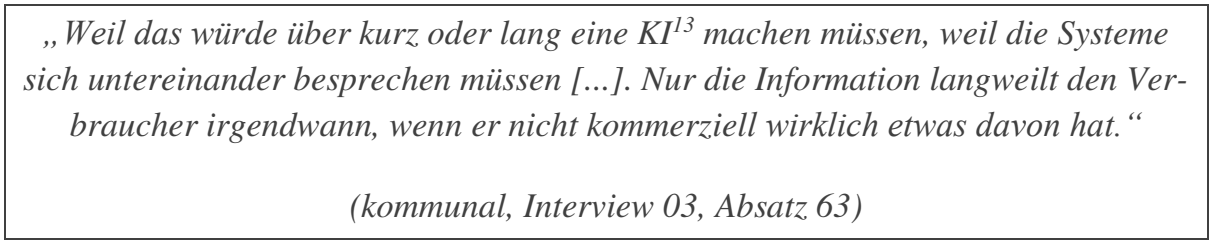

Vorzuziehen sei ein vollautomatisierter Prozess, der auch ohne die Aufmerksamkeit des Kunden im Hintergrund agiere, nachdem dieser einmalig konfiguriert wurde. Die Handlungen müssten dabei monetäre Auswirkungen haben in Form von Einsparungen/Erlösen oder Mehrausgaben/Mindereinnahmen.

$$
\begin{gathered}
\text {,[...] eine schöne App und da kann er ja sofort sehen, welches Gerät angeschaltet } \\
\text { wurde. [...] Wenn man ihm aufzeigen kann: „Pass auf, du hast den Strom heute wie- } \\
\text { der verkauft und 3,80 Euro bekommen. “ Dann freut er sich [...]." } \\
\text { (kommunal, Interview 03, Absatz 65) }
\end{gathered}
$$

Um ein Bewusstsein für Strom zu schaffen könne eine Visualisierung des aktuellen Stromverbrauchs und der Stromerzeugung unterstützen. Es wird jedoch von zwei der fünf Interviewpartner darauf hingewiesen, dass eine alleinige Visualisierung ohne monetäre Auswirkungen nicht ausreiche. Mit Energiemengen oder Leistungskurven seien die meisten Kunden zudem überfordert oder sie möchten sich nicht damit auseinandersetzen. Daher seien diese Informationen höchstens kurzfristig interessant, verlören jedoch schnell ihren Reiz und führten nicht zu einer langfristigen Verhaltensänderung.

$$
\begin{gathered}
\text { „Ja, das sind für den nur Zahlen. [...] Es ist interessant am Anfang, aber dann lässt } \\
\text { es nach. " } \\
\text { (kommunal, Interview 03, Absatz 65) }
\end{gathered}
$$

Der vierte Experte, der sich zu lokalen Handelsplätzen äußert, sieht vor diesem Hintergrund ein Aggregatormodell ${ }^{14}$ als vielversprechend an, da es die Komplexität des Handels reduziere und somit dem Kundenwunsch nach Einfachheit gerecht werde.

$$
\begin{gathered}
\text { „Ich glaube, dass die einfachste Form des Marktplatzes wäre: Es gibt einen Aggre- } \\
\text { gator der Erzeuger und Verbraucher jeweils aggregiert. " } \\
\text { (bürgernah, Interview 09, Absatz 38) }
\end{gathered}
$$

Der fünfte Experte weißt im Zusammenhang mit der Umsetzung eines lokalen Handelsplatzes darauf hin, dass der Community-Aspekt nicht außer Acht gelassen werden dürfe, da dieser in jüngster Vergangenheit an Bedeutung gewinne, insbesondere in urbanen Räumen (siehe Kapitel 5.3.3).

\footnotetext{
${ }^{13}$ KI beschreibt ein Teilgebiet der Informatik, das sich mit der Erforschung und Entwicklung sog. ,intelligenter Agenten“ befasst, die selbstständig Probleme lösen können. Eine allgemeingültige, tiefergehende Definition existiert nicht [84, S. 6].

${ }^{14}$ Der Begriff Aggregatormodell beschreibt in diesem Kontext ein Modell, bei dem ein Dienstleister (Aggregator) die Beschaffung und Veräußerung des Stroms in einem lokalen Marktplatz übernimmt. Der Kunde besitzt je nach Modell gewisse Eingriffsmöglichkeiten, z.B. die Eingabe von Präferenzen.
} 
„Ja auch dieser Community-Aspekt ist ja dabei nicht zu verachten, der um sich greift. Ja auch in Großstädten erlebe ich das zunehmend mehr. “

(regional, Interview 17, Absatz 20)

Weiterhin besteht seitens eines Experten der Wunsch, dass es in einem lokalen Energiemarkt möglich sein solle, die Stromlieferung auch ohne Herkunfts- und Regionalnachweisregister - zumindest in seiner jetzigen Ausgestaltungsform - nachzuweisen.

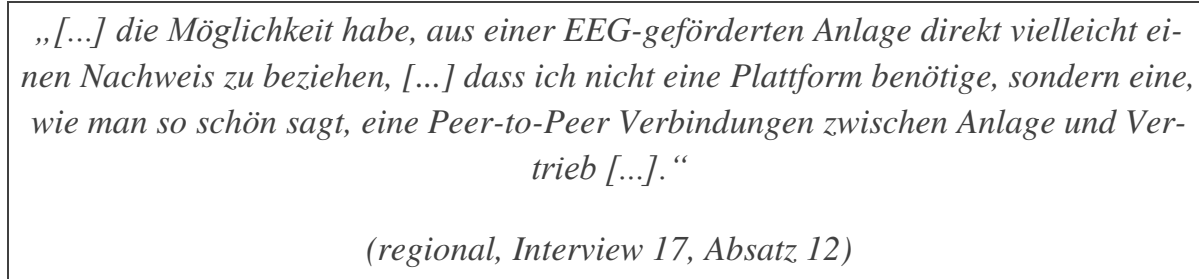

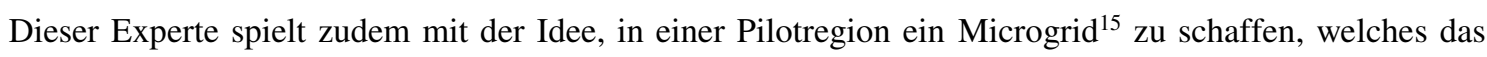
übergelagerte Netz ggf. nur noch zur Frequenzhaltung benötigt. Das Microgrid wäre ansonsten autark.

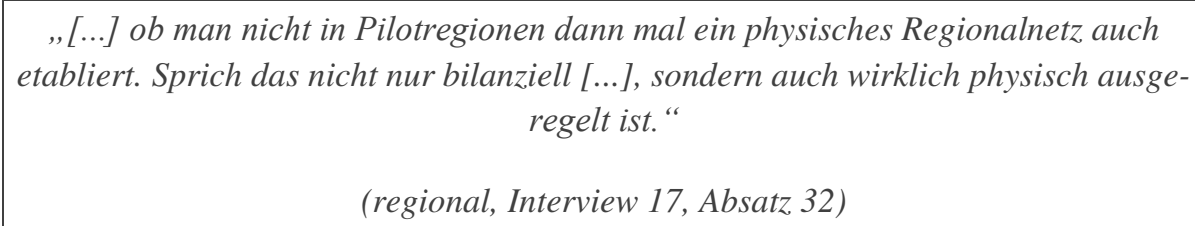

Das Konzept Regionalstrom könne zudem zukünftig mit einer finanziellen Beteiligung der Kunden verbunden werden (siehe Kapitel 5.3.2). Ein Experte nennt in diesem Zusammenhang die Beteiligungsmöglichkeit an einem Quartiersspeicher. Dies habe nicht nur den Vorteil, dass eine Beteiligung nicht vom Vorliegen gewisser Rahmenbedingungen beim Kunden abhänge (z.B. Kunde ist Eigentümer eines Hauses mit ausreichender Dachfläche und Platz im Keller), sondern Größendegressionseffekte könnten gehoben werden. Letzteres fördere die Wirtschaftlichkeit und damit Attraktivität von Investitionen.

„Also wir geben [...] mit diesem Kleinteiligen schon sehr viel mehr Geld aus für
eine Energiewende, als wenn das Ganze in einer strukturierten Art und Weise statt-
finden würde, dass man einfach auch wieder in größeren [...] Quartierseinheiten
denken würde [...]. Also da sehe ich eher noch die sinnvolle Chance, als zu klein,
klein zu werden."
(kommunal, Interview 06, Absatz 64)

Die von dem Experten vorgeschlagene Beteiligung in Form einer Miteigentümerschaft kann jedoch trotz wirtschaftlicher Vorteile mit dem Nachteil einhergehen, weniger akzeptiert zu werden, da sich das Investment nicht im alleinigen Eigentum und nicht im Besitz des Kunden befindet. Letzteres ist in der Verhaltensökonomik als Besitztumseffekt ${ }^{16}$ bekannt.

\footnotetext{
${ }^{15}$ Unter einem Microgrid (deutsch: Inselnetz) ist ein lokales, abgegrenztes Stromnetz zu verstehen.

${ }^{16}$ Als Besitztumseffekt (englisch: endowment effect) wird der Umstand beschrieben, dass der (Nicht-) Besitz eines Gutes einen Einfluss auf dessen Bewertung hat. Personen, die im Besitz eines Gutes sind, bewerten dieses regelmäßig wesentlich höher als Personen, die nicht im Besitz des Gutes sind [85].
} 


\subsubsection{Post-EEG-Anlagen}

Hinsichtlich der Frage, welche Auswirkungen das Ausscheiden der ersten EEG-Anlagen 2021 auf das Konzept Regionalstrom haben wird, sehen alle Experten einen Handlungsbedarf bei der Vermarktung der Strommengen aus diesen Anlagen. Für die Post-EEG-Anlagen gibt es drei mögliche Handlungsoptionen: (1) Weiterbetrieb (2) Repowering oder (3) Abbau der Anlage. Bei den Optionen (1) und (2) besteht die Möglichkeit zur Netzeinspeisung, zum Eigenverbrauch oder einer Kombination aus beidem.

"Letztendlich ist ja die Thematik: Was macht der Anlagenbetreiber mit der Anlage?
Baut er sie ab? Kann er sie repowern? Oder vermarktet er den Strom?"
(kommunal, Interview 12, Absatz 70)

Die Anlagen, welche ab 2021 aus der EEG-Förderung ausscheiden, werden laut drei Experten - sollte sich an der jetzigen Regulatorik nichts ändern - aufgrund ihrer geringen Erzeugungsleistungen kaum einen Einfluss auf das Konzept Regionalstrom haben.

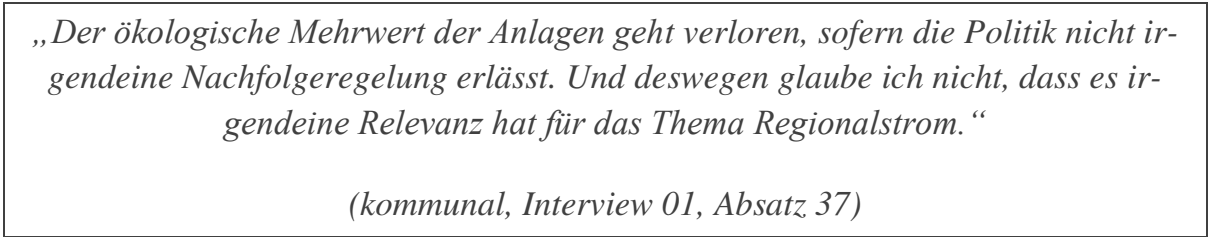

Da es sich bei den ausscheidenden Anlagen zunächst um Klein- und Kleinstanlagen handle, sei eine proprietäre Anbindung dieser Anlagen an einen Aggregator (z.B. Stadtwerk) und ggf. eine Anmeldung beim HKNR, die aktuell je Anlage individuell durchgeführt werden müsse und mit zusätzlichen Gebühren einhergehe, allein aufgrund des operativen Mehraufwands kaum wirtschaftlich darstellbar.

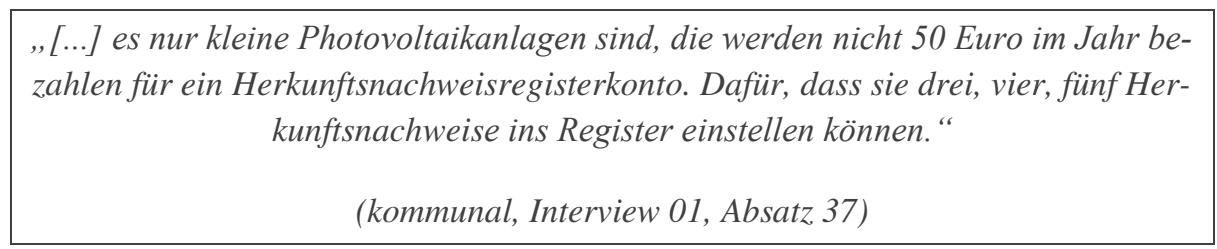

Ein Experte eines kommunalen Versorgers nennt als eine mögliche Fallback-Option den Netzbetreiber zur Aufnahme der Strommengen der Post-EEG-Anlagen zu verpflichten, jedoch ohne Vorrangregelung der Einspeisung. Letzteres entspreche eine Neuerung zum aktuellen Einspeisevorrang des EEG (§ 11 EEG). Die Anlagenbetreiber bekämen in diesem Fall (nur) den Marktwert des Stroms gutgeschrieben. Diese Lösung könne für all diejenigen Anlagenbetreiber interessant sein, die keinen alternativen Dienstleister für die Vermarktung suchen möchten oder finden. Werde regulatorisch keine Fallback-Option geschaffen, würden die Erzeugungsmengen der Post-EEG-Anlagen nicht mehr erfasst und der ökologische Mehrwert gehe im System verloren. Darauf weist dieser Experte hin.

Das Ausscheiden größerer Anlagen aus der EEG-Förderung, z.B. Windkraftanlagen, eröffne den Vertrieben die Möglichkeit, den Anteil des Stroms aus der sonstigen Direktvermarktung nach § 21a EEG bei einem Regionalstromprodukt leichter und zu geringeren Kosten zu erhöhen. Sollte bislang auf Anlagen aus dieser Veräußerungsform zurückgegriffen werden, mussten diese laut Experten sehr teuer aus der EEG-Förderung 
„herausgekauft“ werden (siehe Kapitel 5.1 und 5.2). ${ }^{17}$ Weiterhin bietet es sich laut Experten an, für die Erzeugungsmengen der aus dem EEG ausscheidenden Anlagen Herkunftsnachweise ausstellen zu lassen, sofern die Kosten der Registernutzung die Erlöse der HKN-Vermarktung nicht übersteigen. Die HKN können genutzt werden, um den Nachweis der Grünstromeigenschaft zu erbringen. Weiterhin kann je nach Anlagenstandort mit der Regionalität der Erzeugung geworben werden (siehe Kapitel 2.5).

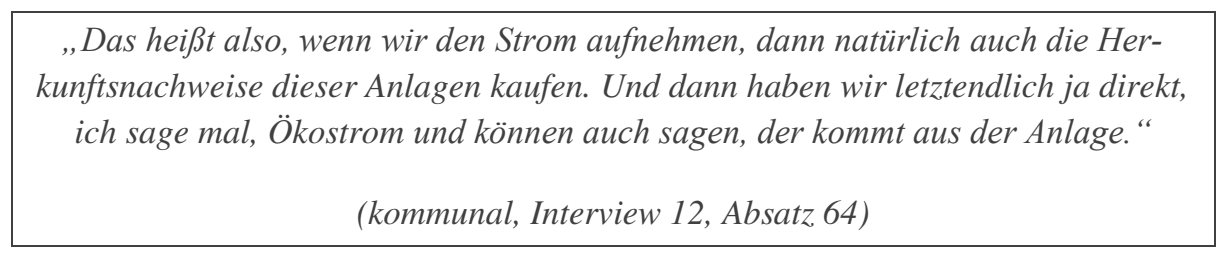

Vor dem Hintergrund der (vergleichsweise günstigen) Nutzung von Post-EEG-Anlagen für ein Regionalstromprodukt, sofern die Anlagenleistung ausreichend hoch ist, sind drei Experten der Auffassung, dass das Regionalnachweisregister in Zukunft an Bedeutung verlieren werde.

„, [...] wenn die Anlagen aus dem EEG fallen, dann eventuell das Regionalnachweis-
register gar nicht mehr so angesagt ist und wir den regionalen Strom eben auch aus
den Anlagen bekommen, die nicht mehr im EEG sind."
(bürgernah, Interview 05, Absatz, 55)

Wird auf eine Nutzung des Regionalnachweisregisters verzichtet, so gehe dies unter anderem mit dem Vorteil einher, nicht mehr an dessen Kriterien, insbesondere hinsichtlich Regionalität, gebunden zu sein (siehe Kapitel 2.5).

„Denn sobald die Anlagen aus der Förderung fallen habe ich ja erstmals die Situa-
tion, [...] dass ich Herkunftsnachweise für diese Anlagen erhalten kann beziehungs-
weise ohne regulatorische Hürden diesen Strom an Endverbraucher liefern kann in
der jeweiligen Region. "
(bürgernah, Interview 09, Absatz 44)

Für den Weiterbetrieb von Post-EEG-Anlagen gilt es jedoch zu beachten, dass eine Mindestvergütung in Abhängigkeit des Anlagentyps notwendig ist, um deren variablen Kosten zu decken. Die Kosten dürfen für einen Weiterbetrieb den Marktpreis für Strom bzw. die Zahlungsbereitschaft der Endkunden für Regionalstrom nicht übersteigen.

Bei Kleinstanlagen, insbesondere Photovoltaik, lohne sich eine ausschließliche Netzeinspeisung für den Betreiber kaum, da der Marktwert des Stroms zu gering sei. Die finanziellen Einsparungen seien in diesem Fall bei Eigenverbrauch die höchsten, wobei zu beachten sei, dass dies häufig mit einem Umbau der Messinfrastruktur vor Ort einhergehe. Die Kosten des Umbaus könnten die potenziellen Einsparungen übersteigen.

\footnotetext{
${ }^{17}$ Eine alternative Möglichkeit stellt die Nutzung von Erneuerbare-Energien-Anlagen dar, die bereits vor der Einführung des EEG in Betrieb genommen wurden. Diese besitzen i.d.R. keinen Förderanspruch und befinden sich somit in der sonstigen Direktvermarktung nach § 21a EEG. Die Verfügbarkeit solcher Anlagen ist jedoch regional sehr unterschiedlich (z.B. Wasserkraftanlagen).
} 


$$
\begin{aligned}
& \text { „Da muss er seine Anlage umbauen, muss die ganze Zählermimik ändern und so. } \\
& \text { Das sind Tausende von Euros womöglich, die er in die Hand nehmen muss, wegen } \\
& \text { einer kleinen Einsparung die er da hat." } \\
& \text { (kommunal, Interview 13, Absatz 79)“" }
\end{aligned}
$$

Werde der Eigenverbrauch maximiert, so lasse sich zudem extrem schwer prognostizieren, welche Restmengen noch in das öffentliche Netz eingespeist werden. Da die Abnahme des Stroms aus Kleinstanlagen bereits ohne Eigenverbrauch kaum einen Business Case darstelle, werde die Wirtschaftlichkeit in diesem Fall noch weiter erschwert, da die Einspeisemengen noch geringer seien und das Risiko von Bilanzkreisabweichungen steige.

$$
\begin{aligned}
& \text { „Und die Mengen sind so klein und so unbedeutend. Und dann betreibt er womög- } \\
& \text { lich noch Eigenverbrauch. Dann weiß ich erst recht nicht was dann ins Netz rein- } \\
& \text { fließt an Überschussstrom." }
\end{aligned}
$$

(kommunal, Interview 13, Absatz, 79)

Zusammenfassend ist davon auszugehen, dass das Ausscheiden von Anlagen aus dem EEG in Abhängigkeit von der jeweiligen Region unterschiedliche Auswirkungen auf das Konzept Regionalstrom haben wird. Im windreichen Norden mit vergleichsweise großen Anlagenleistungen wird der Zugriff auf diese Post-EEGAnlagen einfacher und wirtschaftlicher sein als im sonnenreichen Süden mit zahlreichen Klein- und Kleinstanlagen.

Der aktuelle Referentenentwurf des BMWi zur Änderung des EEG ab dem 01. Januar 2021 [29] sieht vor, dass der Einspeisevorrang der ausscheidenden Erzeugungsanlagen erhalten bleibt. Weiterhin soll es Anlagenbetreibern ermöglicht sein, den Strom wie bisher in der Veräußerungsform der sonstigen Direktvermarktung selbst zu vermarkten oder den Strom bis Ende 2027 dem Netzbetreiber zur Verfügung zu stellen. In letzterem Fall sollen die Anlagenbetreiber den Marktwert des Stroms abzüglich der Vermarktungskosten erhalten. Diese Vermarktungsvariante ist insbesondere für Anlagen mit geringer Erzeugungsleistung gedacht, deren Weiterbetrieb in der sonstigen Direktvermarktung unter Umständen derzeit nicht wirtschaftlich ist.

Die Einschätzung der Experten, dass sich eine proprietäre Anbindung und Direktvermarktung von Kleinanlagen nicht lohne und daher keine Direktvermarktungsanbieter auf den Markt träten, spiegelt nicht das aktuelle Marktgeschehen wider [86]. Es lässt sich jedoch nicht beurteilen, ob die zunehmende Anzahl an Direktvermarktungsanbietern für Post-EEG-Anlagen auf tatsächlich realisierbare Gewinnmargen oder auf eine Pionierstrategie zurückzuführen ist. Letztere bietet für den First-Mover ${ }^{18}$ auf einem Markt hohe Chancen, jedoch auch hohe Risiken [88, S. 246].

\footnotetext{
${ }^{18}$ Unter dem First Mover Advantage wird der Vorteil verstanden den ein Unternehmen hat, wenn es als erstes mit einem neuen Produkt am Markt erscheint. Dies kann entweder durch eine Produktinnovation geschehen oder durch ein bereits etabliertes Produkt, das in einem neuen Markt eingeführt wird [87, S. 13].
} 


\subsection{Umsetzbarkeit durch Versorgerklassen}

Aktuell beschäftigen sich nicht nur regionale, kommunale und bürgernahe Versorgungsunternehmen mit dem Thema Regionalstrom, sondern auch überregional agierende Versorger (siehe Kapitel 4.1). Fünf Interviewpartner weisen hinsichtlich der Frage, für welche Klasse von Versorger das Produkt Regionalstrom am besten umzusetzen ist, darauf hin, dass generelle Aussagen schwierig seien.

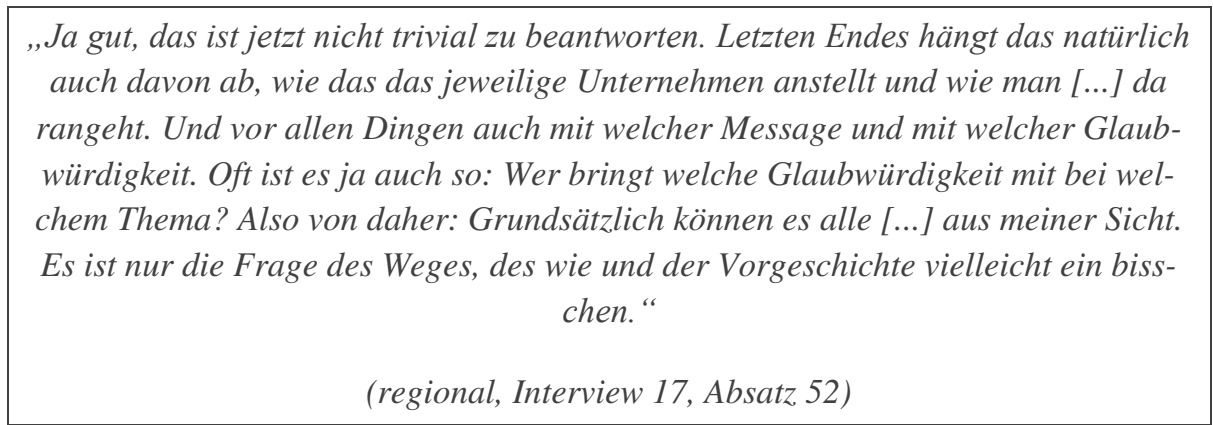

Denn einerseits sei die beste Umsetzbarkeit von Region zu Region unterschiedlich.

$$
\begin{gathered}
\text { „Ich glaube zum einen, dass es Regionen geben wird, wo das besser läuft als in an- } \\
\text { deren. “ } \\
\text { (bürgernah, Interview 07, Absatz 34) }
\end{gathered}
$$

Andererseits spiele die Authentizität eines Versorgers eine starke Rolle. In diese fließen laut Experten Faktoren wie die bisherige Positionierung eines Versorgers, die Produktaussagen, die Nahbarkeit und viele weitere Faktoren ein. Zudem könne sich als First-Mover in einer Region ein langfristiger Wettbewerbsvorteil ergeben. Nachfolgend werden die Aussagen der Experten zu den einzelnen Versorgerklassen (siehe Kapitel 4.1) dargelegt.

\subsubsection{Kommunale Energieversorger}

Ausnahmslos alle Interviewpartner sind der Auffassung, dass das Image der kommunalen Versorgungsbetriebe und die Story eines Regionalstromprodukts stimmig seien. Insofern lasse sich ein Regionalstromprodukt leichter bewerben, bspw. mit regionaler Wertschöpfung und der Präsenz des Unternehmens vor Ort.

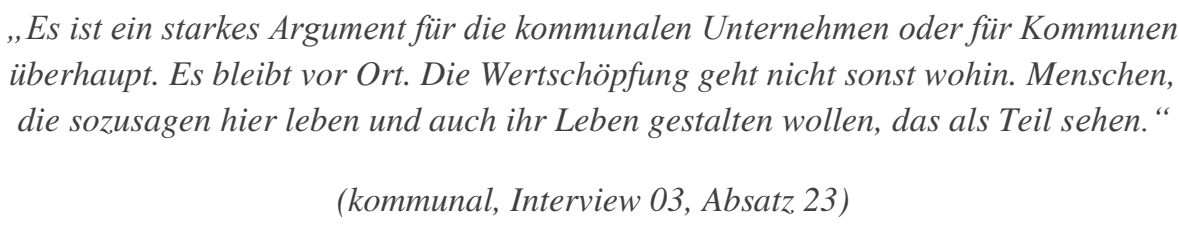

(kommunal, Interview 03, Absatz 23)

Ein Experte sieht einen weiteren Vorteil im Kundenstamm der kommunalen Versorger, der zugänglicher für ein regionales Stromprodukt sei, während er den Kundenstamm der überregionalen Versorger als preissensitiver einstuft.

Doch auch auf der Beschaffungsseite berichten acht Interviewpartner von Vorteilen: Häufig befänden sich für Regionalstrom geeignete Anlagen im Eigentum des jeweiligen kommunalen Versorgungsunternehmens oder mit diesen bestünden Direktvermarktungsverträge. Selbst wenn beides nicht zutreffe, falle die Kontaktaufnahme leichter, da die notwendigen Kontaktdaten vorlägen oder leichter zugänglich seien. 
„Ich glaube aber, dass den Vorteil eher so die kleineren regionalen Versorger haben oder EVUs, die letztlich auch überwiegend Kontakt zu den Anlagenbetreibern haben vor Ort. Daher auch eine Möglichkeit oder die bessere Möglichkeit haben, diese Anlagen zu akquirieren, also [...] die Herkunfts- oder die Regionalnachweise darüber zu akquirieren."

(kommunal, Interview 12, Absatz 66)

\subsubsection{Bürgernahe Energieversorger}

Fast alle Interviewpartner sehen für die bürgernahen Versorger die gleichen Vorteile wie jene der kommunalen Versorger. Dazu zählen die Kongruenz zwischen dem Image des Unternehmens und den Produktaussagen sowie die Verknüpfung der Produktaussagen mit regionaler Wertschöpfung und der Präsenz des Unternehmens vor Ort. Sofern keine Neidgedanken vorliegen, sieht ein Interviewpartner einen Marketingvorteil in der Nähe zwischen den Bürgern, die sich in einer Mund-zu-Mund-Propaganda materialisiere.

$$
\begin{aligned}
& \text { „Dann gehen diese Gesellschafter an Friends and Family und sagen: „Pass mal } \\
& \text { auf, du nimmst mal bitte unseren Strom. “ Dann ist da auch der Zugriff leicht. Das } \\
& \text { heißt du hast schon einen Marketingvorteil." } \\
& \text { (regional, Interview 16, Absatz 26) }
\end{aligned}
$$

Dennoch berichten sechs Interviewpartner von Nachteilen, mit denen kleine, bürgernahe Versorger gegenüber kommunalen Versorgern zu kämpfen haben: Sie könnten u.a. die Anforderungen, die an ein EVU in Deutschland gestellt werden, meist nicht alleine operativ und kompetenzseitig stemmen.

$$
\begin{gathered}
\text { „Selbst EVU zu sein, also Energieversorgungsunternehmen. Das ist in Deutschland } \\
\text { mit sehr viel Auflagen und Anforderungen verbunden, die richtig was kosten." } \\
\text { (bürgernah, Interview 02, Absatz 16) }
\end{gathered}
$$

Fünf Interviewpartner empfehlen daher, in diesen Fällen auf einen Dienstleister zurückzugreifen, der die operative Abwicklung übernimmt. Ein bürgernaher Experte schildert zudem das Problem, dass kleine, bürgernahe Versorger geringere Portfolioeffekte im Erzeugungspool hätten.

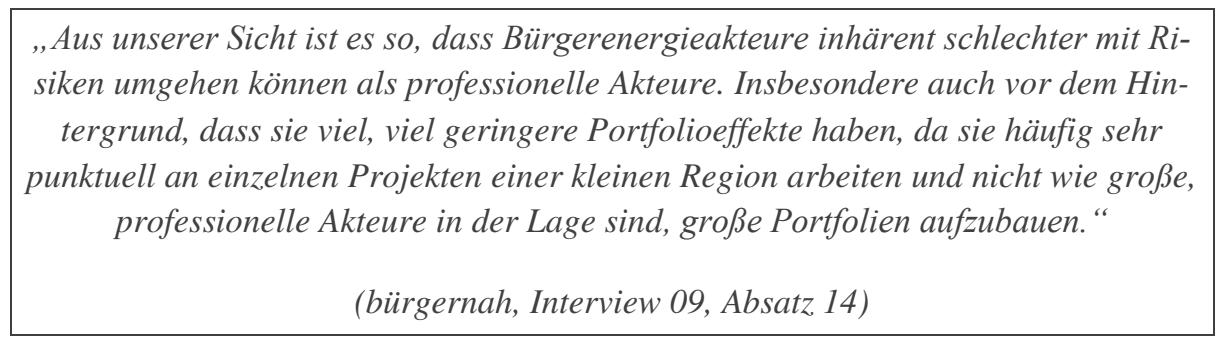

Energieversorgungsunternehmen sind in Deutschland dazu verpflichtet, ihre Kunden viertelstundenscharf mit Elektrizität aus ihrem Bilanzkreis zu beliefern. Bei einer Belieferung mit Regionalstrom gilt diese Anforderungen nicht zwangsläufig für die regionale Komponente, sondern es kann bspw. ein mengenmäßiger Abgleich zwischen der regional erzeugten und verbrauchten Strommenge über einen Bilanzierungszeitraum erfolgen (siehe Kapitel 2.5). Nehmen Bürgerenergiegesellschaften selbst die Rolle eines EVU wahr, so müssen sie diese Anforderungen erfüllen. 
Ein Experte empfiehlt daher den Bürgerenergiegesellschaften, lediglich die Erzeugerrolle in der Energiewirtschaft wahrzunehmen und den regional erzeugten Strom an andere Unternehmen, bspw. ein Stadtwerk, zu verkaufen.

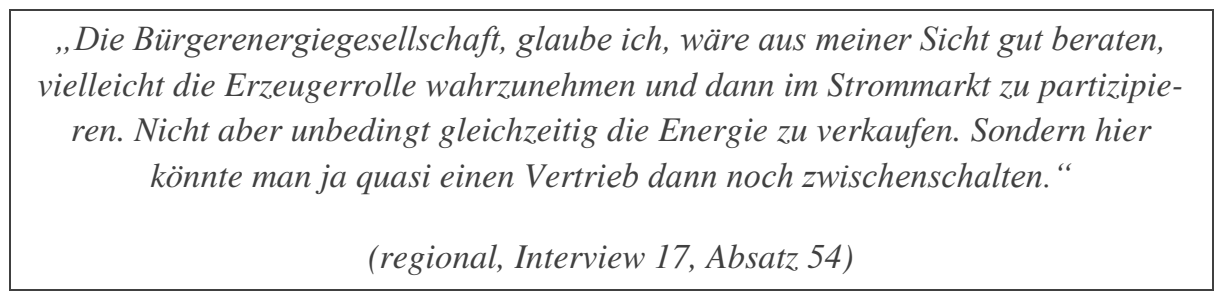

\subsubsection{Regionale Energieversorger}

Die bei den kommunalen Versorgungsunternehmen genannten Vorteile auf der Vertriebs- und Beschaffungsseite gelten laut Experten näherungsweise auch für regionale Versorger. Jedoch ergäben sich Unterschiede aus der Größe der Versorgungsgebiete. Sind die Gebiete zu groß, könnten regionale Versorger gegenüber kommunalen Versorgern ein Regionalstromprodukt ggf. schlechter bewerben, da die Produktaussagen und das Unternehmensimage immer weniger zusammenpassten. Darauf weisen zwei Interviewpartner hin.

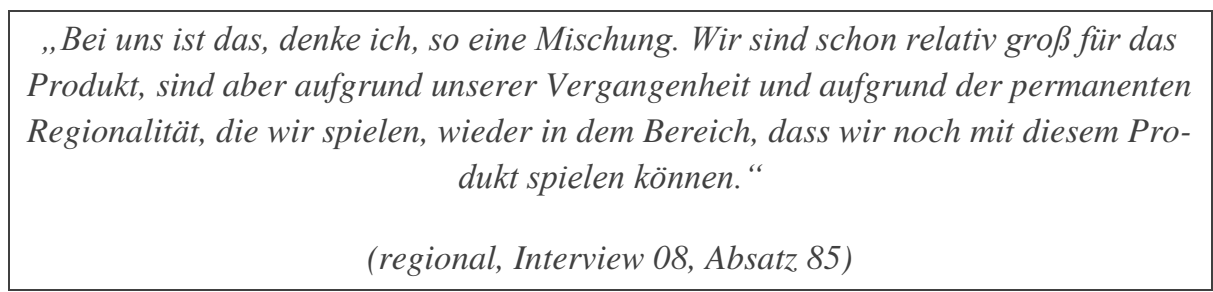

Allerdings könnten kommunale Versorger, deren Versorgungsgebiet klein ist, nur Strom aus Anlagen vermarkten, die sich in diesem kleinen Gebiet befinden. So schildert ein kommunaler Experte, dass er den Strom aus Windrädern in seiner Nachbargemeinde nicht als Regionalstrom vermarkten könne, da der Regionenbezug zwischen Unternehmen und Produkt nicht gegeben sei.

„Ich könnte nie als Partner für diese vierzig Windräder auftreten und die regional
vermarkten. Ein großes [regionales Energieversorgungsunternehmen], die 300.000
Kunden in der Region versorgt, hat natürlich diese Option schon eher.

(kommunal, Interview 06, Absatz 60)

Hier seien die regionalen Versorger im Vorteil, da sie tendenziell auf mehr Anlagen aus ihrer größeren Region zurückgreifen könnten und dennoch der Regionenbezug gegeben sei.

\subsection{4 Überregionale Energieversorger}

Nicht alle Interviewpartner schließen eine Aufnahme von Regionalstromprodukten in das Portfolio der überregionalen Versorger aus. Allerdings gestalte sich für die überregionalen Versorger die Informationsbeschaffung und das Marketing in den jeweiligen Vertriebsgebieten schwieriger, bspw. um Zielgruppenanalysen durchzuführen oder das Vertriebsmaterial anzupassen (siehe Kapitel 5.3.3). Eine weitere Hürde stelle die Authentizität dar. Diese sei eventuell nicht gegeben, sodass die Produktaussagen nicht zum Image des jeweiligen Unternehmens passten. Das Problem der mangelnden Authentizität könne eventuell 
umgangen werden, indem Submarken gegründet werden und somit neue Player den Markt für Regionalstrom betreten.

,Ich glaube aber auch, dass es große Akteure geben wird, die das sozusagen emu-
lieren durch entsprechende Submarken oder Subplattormen, die sie dann regional
betreiben."
(bürgernah, Interview 09, Absatz 46)

Zwei Interviewpartner sind der Auffassung, dass urbane Räume ein attraktives Vertriebsgebiet für die überregionalen Versorger darstellten, sofern Erzeugungsanlagen vorhanden und der Zugriff auf diese gegeben sei, z.B. über bestehende Direktvermarktungsverträge oder vorangegangene Geschäftsbeziehungen. Allerdings könnten auch regional verankerte Versorger die urbanen Räume als attraktive Absatzgebiete für Regionalstrom identifizieren, was den Wettbewerb erhöhe.

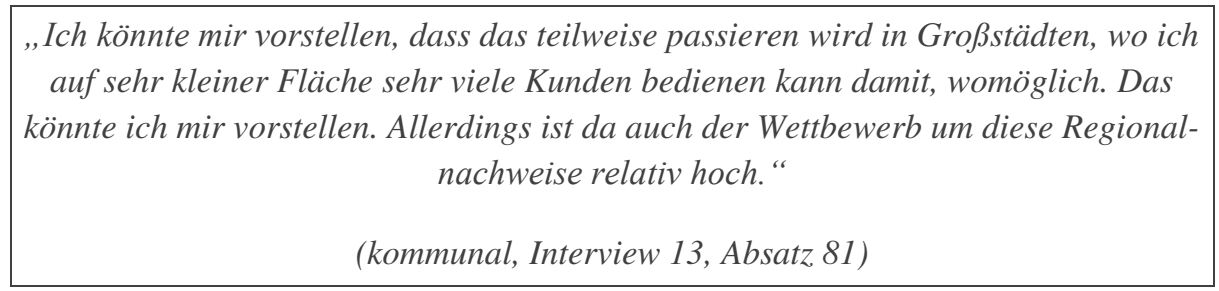

Auf der Beschaffungsseite müssen die überregionalen Versorger laut Experten ebenfalls Hürden meistern: Sofern keine Direktvermarktungsverträge mit den jeweiligen Anlagen vor Ort bestehen oder sich die Anlagen im Eigentum des überregionalen Versorgers befinden, muss der Kontakt zu Anlagenbetreibern bzw. Vermarktern hergestellt werden (siehe Kapitel 5.2.2). Häufig lägen diese Kontaktinformationen nicht vor (siehe Kapitel 5.1.1). Weiterhin gibt ein Interviewpartner zu bedenken, dass die Akzeptanz für Anlagen von überregionalen Versorgern tendenziell geringer sei als die Akzeptanz für Anlagen von kommunalen oder bürgernahen Versorgern, da der Kunde bei Letzteren davon ausgehe, dass die Wertschöpfung in der Region bleibe.

\begin{tabular}{|} 
„Da geht es darum: Welche Akteure treten auf? Wem gehört das Ganze danach? \\
Wo fließt die Wertschöpfung hin? Und wenn ich dafür gute Antworten habe, sei es \\
als Genossenschaft, sei es als Stadtwerk oder sei es vielleicht sogar zu überregiona- \\
ler Versorger. Wenn ich da einen guten Prozess für habe und die Menschen wirklich \\
mit einem ehrlichen Interesse, sie zu beteiligen, mitnehme. Dann glaube ich, dass " \\
ich eigentlich in der Gesamtschau der Gesellschaft im Image nur gewinnen kann." \\
(bürgernah, Interview 09, Absatz, 26)
\end{tabular}

Unter Berücksichtigung der Schwierigkeiten, mit denen bundesweit agierende Versorgungsunternehmen zu kämpfen haben, sind zwei Experten der Auffassung, dass diese das Thema Regionalstrom - analog zu den heute bereits weit verbreiteten Ökostromtarifen - nur gewinnen können, wenn sie dem Kunden einen Preisvorteil offerieren. Dies bedinge allerdings Kosteneinsparungen gegenüber den anderen Versorgungsunternehmen. Mittels IT könnten Prozesse automatisiert und somit kostengünstig abgewickelt werden. In der aktuellen Regulatorik sei eine solche automatisierte Abwicklung jedoch nur schwer möglich (siehe Kapitel 5.2.2). 


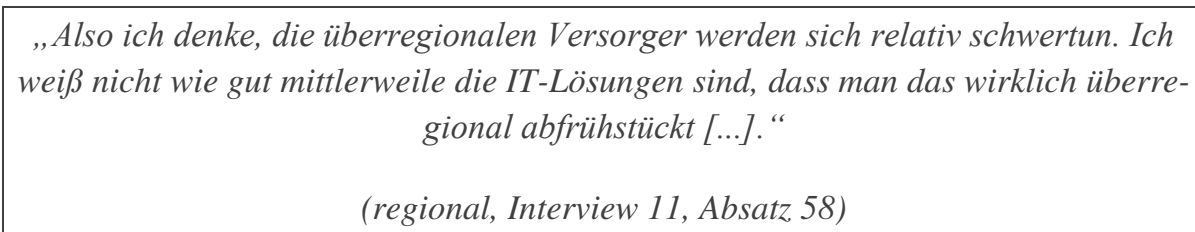

Der Interviewpartner des überregionalen Versorgers bestätigt, dass eine bundesweite Etablierung von Regionalstromprodukten momentan vermutlich nicht wirtschaftlich sei und attraktive Absatzgebiete identifiziert werden müssten. Als Testgebiete böten sich Regionen an, in denen ein bundesweit agierender Versorger bereits breit aufgestellt sei.

„Wir wollen es erst einmal dort spielen wo wir schon viele Kunden haben."

(überregional, Interview 14, Absatz 74)

\section{$5.8 \quad$ Verbesserungsvorschläge}

Eine der abschließenden Fragen aus dem Leitfaden adressierte konkrete Verbesserungsvorschläge, welche sich die Experten für Regionalstrom bei Nutzung des RNR wünschen. Weiterhin werden Verbesserungsvorschläge aufgeführt, die hinsichtlich der Stromkennzeichnung an früheren Stellen in den Interviews genannt wurden. Dies liegt darin begründet, dass nahezu alle Experten Kritik am aktuellen System der Stromkennzeichnung äußerten und Handlungsbedarf auf Seiten der Regulatorik sehen.

\subsubsection{Regionalstrom bei Nutzung des Regionalnachweisregisters}

Als ein wesentlicher Verbesserungsvorschlag werden von drei Experten bürgernaher Versorger wirtschaftliche Vergünstigungen bei Regionalstrom gegenüber überregionalem Strom bzw. Strom unbekannter Herkunft genannt. Dies wird systemisch begründet, insbesondere durch eine geringere Netznutzung.

„Aber was aus meiner Sicht im heutigen System keinen Sinn ergibt ist, dass ich für eine Stromlieferung von hier einmal über die Straße die gleichen Netzentgelte bezahle wie von hier nach [weit entfernte Großstadt] [...]. Grundsätzlich sollte regionaler Strom günstiger sein und könnte es auch sein, wenn man die volkswirtschaftlichen Vorteile in das entsprechende Abgaben- und Entgeltsystem einpreisen würde. "

(bürgernah, Interview 09, Absatz, 12)

Als ein weiterer Grund für Vergünstigungen bei Regionalstrom werden Akzeptanzaspekte auf Seiten der Letztverbraucher genannt (siehe Kapitel 5.3.2). Dabei werde der Umstand adressiert, dass Regionalstromkunden Anlagen in der Region förderten, jedoch für den Flächen- und Landschaftsverbrauch nicht entschädigt würden. Zusammen mit Vergünstigungen werden das Grünstromprivileg (siehe Kapitel 2.4) als attraktive Förderregelung regionalen Grünstroms sowie das Grünstrommarktmodell [37, 89] als potenzielles Nachfolgemodell des Grünstromprivilegs von je zwei Experten kommunaler und bürgernaher Versorger genannt. 
„Also ich meine, wenn man regionalen Grünstrom nutzen und Regionalstrom vertreiben möchte, dann könnten wir schon mal zum Beispiel über das Thema EEG-

Umlage sprechen. Halbieren wir das Thema, so wie früher beim Grünstromprivileg. [...] Und da haben wir auch regionalen Grünstrom verteilt in der Region und auf den Strom einen Nachlass auf die EEG-Umlage erhalten. Ob es dann 50 Prozent sind, ob es ein Cent ist, ob es zwei Cent sind - das ist vollkommen egal. Also einfach nur einen kleinen Benefit mit der Folge hinten raus, dass einfach dieses Produkt in der Region immer günstiger ist als alles andere. “

(regional, Interview 16, Absatz 58)

Jedoch äußert sich ein anderer Experte zum Grünstromprivileg kritisch, da dessen Anforderungen hinsichtlich Regionalität und Kongruenz zwischen Erzeugung und Verbrauch zu anspruchsvoll gewesen seien, weshalb das Unternehmen dieses Experten das Grünstromprivileg kurz nach der Umsetzung wieder eingestellte.

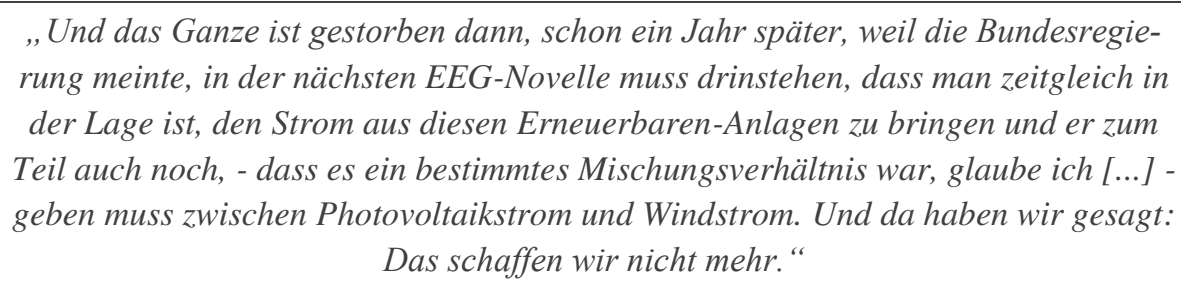
rung meinte, in der nächsten EEG-Novelle muss drinstehen, dass man zeitgleich in der Lage ist, den Strom aus diesen Erneuerbaren-Anlagen zu bringen und er zum Teil auch noch, - dass es ein bestimmtes Mischungsverhältnis war, glaube ich [...] geben muss zwischen Photovoltaikstrom und Windstrom. Und da haben wir gesagt: Das schaffen wir nicht mehr. "

(kommunal, Interview 06, Absatz 4)

Damit adressiert dieser Experte den generellen Nachteil von Regionalstrom, dass Portfolioeffekte auf der Erzeugungs- und Verbrauchsseite mit einer kleiner werdenden Region ebenfalls geringer werden.

Hinsichtlich Verbesserungsvorschlägen zur Regionalitätsdefinition des Gesetzgebers (siehe Kapitel 2.1) ergibt sich unter den Experten kein einheitliches Bild. Drei Experten weisen darauf hin, dass der Kunde vermutlich eine andere Vorstellung von Regionalität habe, wobei zwei dieser Experten einen kleineren Radius als 50 Kilometer nennen.

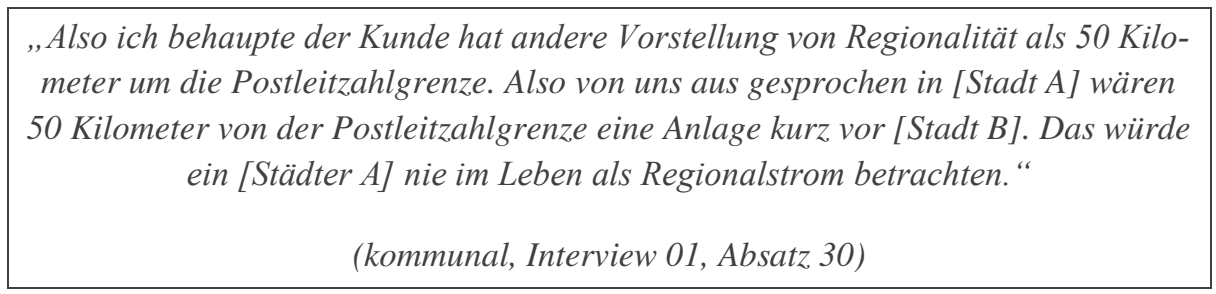

Dennoch sind sich diese Experten auch bewusst, dass es schwierig ist, eine für alle Regionen einheitliche und passende Definition für Regionalität zu finden. Zudem weisen drei Experten darauf hin, dass der vom Gesetzgeber festgelegte Radius bereits für EVU schwierig einzuhalten sei, da teilweise keine Anlagen für ein Regionalstromprodukt zur Verfügung stünden (siehe Kapitel 5.1.1). 


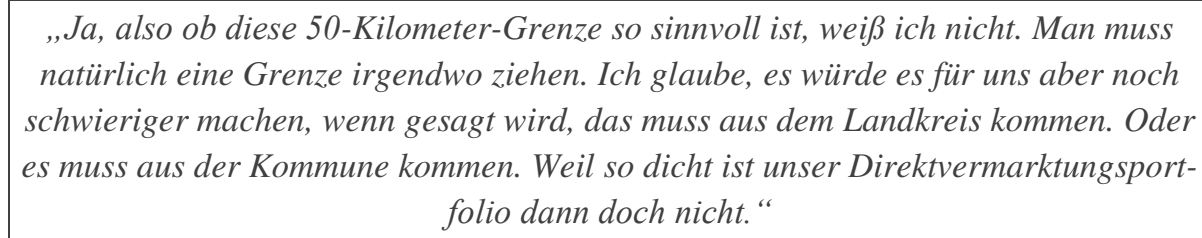

Zusätzlich äußern vier Experten den Wunsch, mittels Regionalnachweisen 100 Prozent Regionalstrom ausweisen zu können (siehe Kapitel 5.4.2).

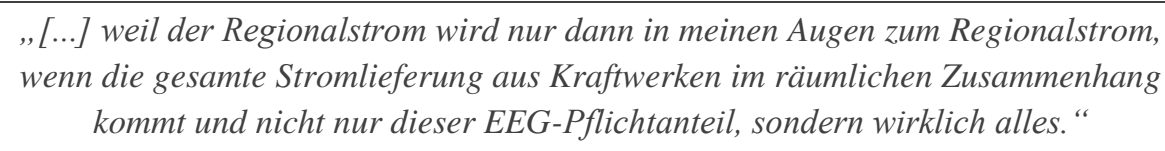

(kommunal, Interview 01, Absatz 10)

Ein Experte eines regionalen Versorgers empfiehlt zudem, Regionalität nicht vom Letztverbraucher ausgehend zu definieren, sondern weitere regionale Stromprodukte zu ermöglichen (z.B. Ostsee-Strom für einen Kunden in Bayern). Eine alternative Möglichkeit für eine 100-Prozent-Ausweisung von Regionalstrom kann laut diesem Experten darin bestehen, zumindest HKN, welche die Anforderungen des RNR hinsichtlich Regionalität erfüllten, ebenfalls für die Ausweisung von Regionalstrom in der Stromkennzeichnung nutzen zu dürfen.

$$
\begin{aligned}
& \text {,[...] also wenn eine einheitliche Definition dann so allgemein, dass man beide } \\
& \text { Nachweise verwenden kann." } \\
& \text { (regional, Interview 08, Absatz 61) }
\end{aligned}
$$

Zuletzt ist laut diesem Experten auch eine Vollerfassung aller Stromerzeugungsmengen inklusive einer Ausstellung von HKN unabhängig von Anlagenart und Förderung denkbar. In diesem Fall würde das HKNR um eine regionale Komponente erweitert und zu einem gewissen Grad das Doppelvermarktungsverbot aufgehoben. Durch die nicht vorgeschriebene Kopplung der HKN an die Stromlieferung (siehe Kapitel 2.3) könnten liquide regionale Marktgebiete für Herkunftsnachweise entstehen.

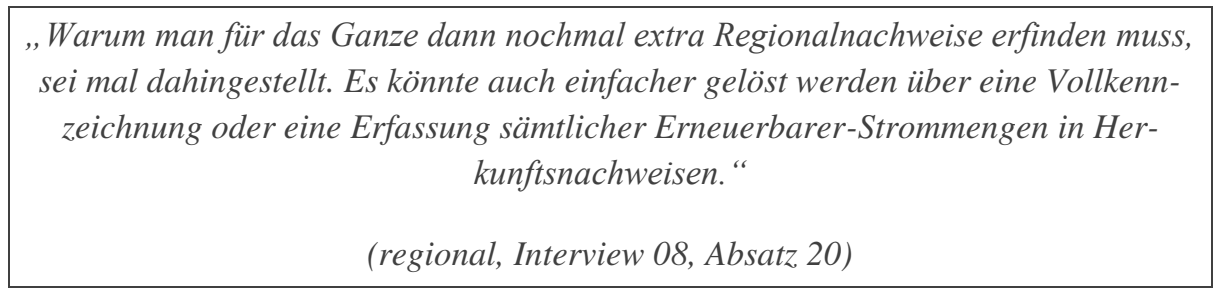

Um die Verständlichkeit für den Endverbraucher (siehe Kapitel 5.4.2) und dessen Vertrauen in Regionalstromprodukte zu erhöhen, könne laut einem Experten eines regionalen Versorgers flankierend zur Stromkennzeichnung ein Regionalstromlabel durch den Staat eingeführt werden, welches bspw. durch das UBA ausgestellt würde. Dies könne die Vielzahl heute am Markt vorherrschender privater Siegel ggf. reduzieren. 
„Da wäre vielleicht auch eine Anregung in Richtung Umweltbundesamt, ob man
sich nicht vorstellen könnte, dass man das jeweilige Regionalstromkonzept als Um-
weltbundesamt zertifiziert. Dass das Umweltbundesamt sagt: „Ich habe mir das an-
geschaut, liebes [Energieversorgungsunternehmen]. Ihr dürft mein Emblem verwen-
den für euer Regionalstromprodukt“. Ist ,, approved by “ oder so. Dann wird der
TÜV sich jetzt auf den Fuß getreten fühlen, weil das ist eigentlich seine Domäne."
(regional, Interview 17, Absatz 14)

Der letzte Verbesserungsvorschlag betrifft die Bürokratie und die manuellen Prozesse bei Nutzung des Regionalnachweisregisters. Hier wünschen sich fünf der 17 Experten aus allen Versorgerklassen Verbesserungen. Es solle eine automatisierte Abwicklung ermöglicht werden, insbesondere durch die Definition von Standards und die Schaffung von Schnittstellen (siehe Kapitel 5.2.2).

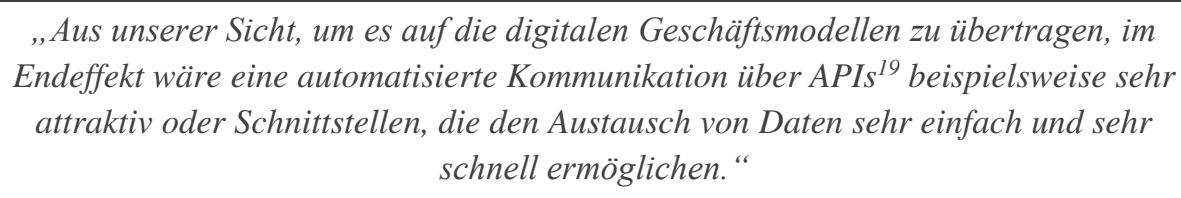

\subsubsection{Stromkennzeichnung}

Die Interviewten sind sich einig, dass die Stromkennzeichnung in ihrer aktuellen Ausgestaltung für den Letztverbraucher nicht verständlich sei. Zwei bürgernahe und ein regionaler Experte kritisieren, dass im heutigen System konventionelle Stromlieferungen durch Zertifikate, insbesondere aus dem Ausland, zu Grünstrom umgewandelt werden können. Es besteht auf Seiten der bürgernahen Versorger der Wunsch nach höheren staatlichen Mindeststandards an Grünstrom und regionalen Grünstrom. Hierdurch könne die Produktdifferenzierung ohne unternehmensspezifisches Marketing erleichtert werden.

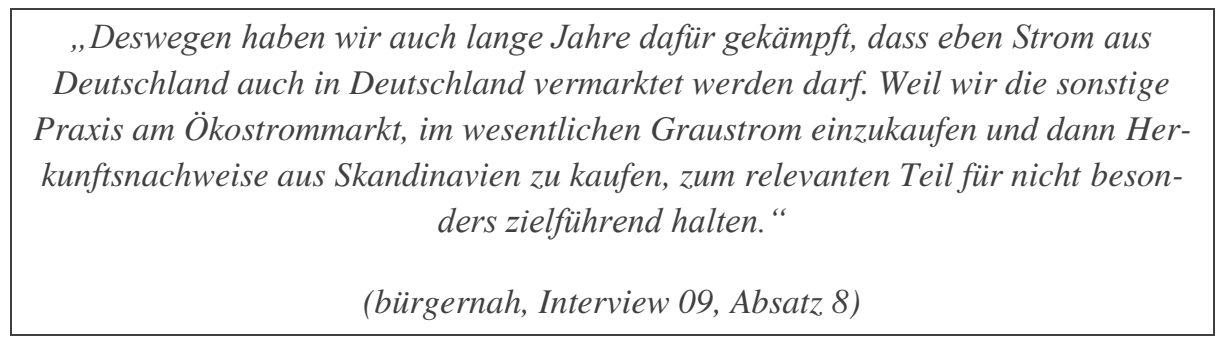

Ein weiterer Kritikpunkt ist die von einigen Interviewpartnern als unzureichend eingestufte Abstimmung zwischen dem EEG-Umlagesystem, welches zur Ausweisung des EEG-Anteils aufgrund der Zahlung der EEG-Umlage seitens der Letztverbraucher berechtigt, und dem HKNR, welches die Grünstellung des verbleibenden Anteils in der Stromkennzeichnung betrifft. Der Letztverbraucher bezahlt im aktuellen System, wenn er Grünstrom beziehen möchte, den EEG-Anteil nochmals in Form von Herkunftsnachweisen mit und wird damit doppelt belastet (siehe Kapitel 2.3). Dieser Umstand wird von drei Experten kommunaler Versorger und einem Experten eines regionalen Versorgers kritisiert.

\footnotetext{
${ }^{19}$ APIs (,application programming interface“, deutsch: Anwendungsprogrammierschnittstelle) bieten die Möglichkeit andere Softwaresysteme über eine Anbindung an ein System anzuschließen.
} 
„Dann mache ich wieder hinterher die EEG-Umrechnung und entwerte quasi den vorher mit Zertifikaten bezahlten Grünstrom gegen das EEG. Ich mache aus Grünstrom einen anderen Grünstrom. Habe aber mit den Zertifikaten vorher trotzdem einen Aufwand gehabt, also habe es bezahlt und abgeführt. Und das ist der Kern meiner Meinung nach des Problems. “

(kommunal, Interview 01, Absatz 10)

Der Umstand, dass bei Ökostrom die EEG-Grünstrommenge (noch einmal) in Form von HKN beschafft werden muss, gehe nicht nur mit einer Doppelbelastung auf Kundenseite einher, sondern könne auch zu Problemen bei der Strombeschaffung führen, bis hin dazu, dass die regionale Stromerzeugung für ein Regionalstromprodukt nicht mehr ausreiche.

\begin{tabular}{|} 
„Dann müsste ich jetzt hergehen und müsste 1000 Megawattstunden Erdgas-KWK \\
zur Verfügung stellen für dieses Stromprodukt. Dann kommt die Umrechnung [...]. \\
Das heißt, 54 Prozent von meinem Erdgasstrom werden quasi grün gestellt -EEG \\
gefördert nach der EEG-Umlage - und dann gehe ich mit meinem Regionalnach- \\
weisregister her und teile diesen Anteil, den ich dann da so ermittelt habe, noch auf \\
meine einzelnen Anlagen auf, entwerte aber faktisch 54 Prozent Erdgas-KWK ins \\
Nirwana. Die sind ja weg. Und wenn ich jetzt hergehe und sage ,,OK, ich mach es \\
anders. Ich mache 40 oder 46 Prozent Erdgas-KWK und der Rest ist halt Graus- \\
trom. "Dann darf ich aber nicht über die Grünstellung aus dem EEG den Graustro- \\
manteil gegen das EEG entwerten. Nein, dann muss ich alles linear entwerten. Das \\
heißt ich habe hinterher wieder ein Graustromkennzeichen drin wo ich wieder \\
Kohle, Atom und sonst was drin habe." \\
(kommunal, Interview 01, Absatz 8)
\end{tabular}

Damit spricht der Experte den Umstand an, dass bei einer Entwertung von HKN kleiner der gelieferten Strommenge die Erzeugungstechnologien anteilig auszuweisen sind (§ 78 Abs. 4 EEG).

Ein Experte eines bürgernahen Versorgers gab abschließend zu bedenken, dass Regionalstrom eine Chance für die Energiewende als Ganzes sei. Denn die Energieversorgung der Zukunft werde vermehrt dezentral ausgestaltet sein, sodass die Nähe zum Versorger ein relevantes Produktmerkmal sei. Solange jedoch die aktuelle Regulatorik bestehen bleibe, bei der Regionalstrom mit finanziellen Nachteilen versehen ist, werde Regionalstrom eine Randerscheinung bleiben (siehe Kapitel 5.6.1). 
„Ich glaube, dass unsere Energieversorgung in Zukunft deutlich regionaler organisiert sein wird. Es fängt an damit, dass das Gebäude zum relevanten Teil Energieerzeuger sein wird. Das heißt, dass ich einen relevanten Teil meines Energieverbrauchs direkt in meinem eigenen Gebäude generiere. Und dann habe ich einen Reststrombedarf oder Restenergiebedarf [...]. Und ich glaube dadurch, dass wir in Deutschland in Zukunft immer mehr dezentrale Anlagen haben werden - es werden Millionen, vielleicht sogar zehn Millionen Anlagen sein -, dass es sehr naheliegend ist, dass man dann nicht einfach irgendein anonymes Kraftwerk hat, [...] sondern dass man diesen Strom in irgendeiner Form aus bekannten Anlagen bezieht, die in irgendeiner Form mit mir in Verbindung stehen. Ob das über den Regionalitätsaspekt geht, ob das über eine Community ist, die mich mit anderen verbindet oder andere Aspekte. Ich glaube, dass die Nähe zur Stromerzeugung in Zukunft ein relevantes Merkmal unseres Energieversorgungssystems und auch damit unseres

Ökostromverbrauchsmodells sein wird. “

(bürgernah, Interview 09, Absatz 42 


\section{Fazit und Ausblick}

Regionalität bei Stromprodukten bzw. -tarifen ist in Deutschland ein aktuelles und teilweise neues Thema. Dies wirft die Frage auf, wie Regionalstrom aus Sicht von Energieversorgern ökonomisch zu bewerten ist. Im Rahmen dieser Arbeit wurden Experteninterviews mit 17 Vertretern von Energieversorgungsunternehmen durchgeführt. Die Interviews zeigen, dass Regionalität bei der Vermarktung von Strom eine Rolle spielt. Damit decken sich die Aussagen der Experten mit jenen der Teilnehmer der vierten Fachtagung des Herkunftsnachweisregisters im Jahr 2016, bei der sieben von elf Energieversorgungsunternehmen angaben, Strom mit dem Zusatz ,regional“" anzubieten [16].

Die Beschaffung von Regionalstrom, entweder durch eigene Erzeugungsanlagen oder durch den Einkauf von Strom aus regionalen Erzeugungsanlagen, gestaltet sich ggü. einer deutschlandweiten Beschaffung als schwieriger. Das Regionalnachweisregister bietet die Möglichkeit, den potenziellen Pool an Erzeugungsanlagen, der für die Umsetzung des Konzepts Regionalstrom genutzt werden kann, zu erweitern. Dennoch geht eine regionale Beschaffung, ob mit oder ohne Regionalnachweisregister, mit Mehraufwänden und ggf. Mehrkosten einher. Bereits 2018 wurden seitens Energieversorgungsunternehmen ggü. dem Umweltbundesamt Bedenken geäußert, dass Regionalstrom bei Nutzung des Regionalnachweisregisters zu teuer sein könnte [90]. Möchte ein Energieversorgungsunternehmen regionalen Ökostrom unter Nutzung des Regionalnachweisregisters anbieten (siehe Option 4 Kapitel 2.5), fallen Nachweis- und Umlagekosten für $211,22 \%$ der gelieferten Strommenge an. Diese Kosten ergeben sich aus 100\% Herkunftsnachweisen, 55,61\% EEG-Umlage und 55,61\% Regionalnachweisen (Stand 2020 für das Bezugsjahr 2018). Auch auf der Vertriebsseite ergeben sich Mehraufwände und Mehrkosten bei Regionalstrom, bspw. für die Erweiterung des Produktportfolios und regionenspezifisches Marketing. Die begrenzte Mehrzahlungsbereitschaft, die vermutlich nur für eine kleine Gruppe an Kunden gilt, in Verbindung mit den Mehraufwänden und Mehrkosten, lässt tendenziell keine höheren Gewinnmargen eines regionalen Ökostromtarifs im Vergleich zu einem herkömmlichen Ökostromtarif zu. Teilweise könnten die Margen sogar negativ sein.

Entsprechend ist nicht davon auszugehen, dass Investitionen in neue Erzeugungsanlagen, die allein auf einer ausreichend hohen und langfristig stabilen Mehrzahlungsbereitschaft fußen, angereizt werden. Auch Hölder und Braig [24] sowie Hauser et al. [1] gehen nicht davon aus, dass Erlöse aus dem Verkauf von Nachweisen, d.h. Herkunftsnachweisen, Regionalnachweisen oder sonstigen Nachweisen ${ }^{1}$, dazu führen, dass Erzeugungsanlagen zugebaut werden. Die vorliegenden Ergebnisse bestätigen die geringe Wirksamkeit freiwilliger Grünstrommärkte auf den Anlagenzubau von [36].

Ein weiteres Ergebnis der Interviews ist, dass neben betriebswirtschaftlich quantifizierbaren Größen aus Sicht von Energieversorgungsunternehmen bei Entscheidungen hinsichtlich Regionalstrom auch nicht-monetäre Größen wie Imagegewinne berücksichtigt werden sollten, die langfristig positiv für ein Unternehmen sein können. Regionalstrom bietet zudem die Möglichkeit zur Unternehmensdifferenzierung [vgl. 90, S. 68]. Weiterhin könnten durch das Anbieten von Regionalstrom - in Abhängigkeit der jeweiligen Region und weiterer Rahmenbedingungen - lokale Widerstände verringert werden, die zu Kosten führen, welche sich jedoch zum Zeitpunkt des Anlagenbaus schwer monetär bewerten lassen. Zuletzt könnten sich zukünftig Verbundeffekte ergeben, sollte der Absatz von Regionalstrom steigen.

\footnotetext{
${ }^{1}$ Das Doppelvermarktungsverbot des $§ 80$ Abs. 2 EEG umfasst nicht nur Regionalnachweise und Herkunftsnachweise, sondern auch sonstige Nachweise, die die Herkunft des Stroms belegen (siehe Kapitel 2.3).
} 
Das Image der kommunalen Versorgungsbetriebe und die Produktaussagen von Regionalstrom sind stimmig. Insofern lässt sich Regionalstrom von diesen Versorgern leichter bewerben, bspw. mit regionaler Wertschöpfung und der Präsenz des Unternehmens vor Ort. Ähnliches gilt für bürgernahe Versorger. Je größer ein Unternehmen bzw. dessen Versorgungsgebiet ist, desto schwieriger wird es, diesen Vorteil auszuspielen. Somit ist es für überregionale Versorger am schwierigsten, Regionalstrom in ihr Produktportfolio mit aufzunehmen. Damit decken sich die Ergebnisse mit denen der EnergieAgentur.NRW aus dem Jahr 2016, welche den Nutzen des Regionalnachweisregisters für überregionale Versorger, Direktvermarkter und Versorger, die vor allem Industrie- und Geschäftskunden bedienen, eher gering einstuft [38].

Allgemeingültige Aussagen hinsichtlich der Auswirkungen von Regionalstrom auf die lokale Akzeptanz sind schwierig [27, S. 116]. Dennoch deuten die Ergebnisse der Experteninterviews sowie die Literatur zur regionalen Grünstromkennzeichnung, bspw. [24, 37, 39], darauf hin, dass bei aggregierter Betrachtung der Einfluss des Regionalnachweisregisters auf das Ziel des Gesetzgebers, die lokale Akzeptanz zu erhöhen, gering sein wird. Dies deckt sich mit den Aussagen der Teilnehmer der vierten Fachtagung des Herkunftsnachweisregisters, die lediglich bei indifferenten Endkunden hinsichtlich regionalen Erzeugungsanlagen von einem positiven Effekt auf die Akzeptanz ausgehen, jedoch nicht bei lokalen Widerständen [16]. Die vielfältigen, regulatorisch zulässigen Vermarktungsvarianten regionaler Stromprodukte bergen zudem die Gefahr von Intransparenz und mangelnder Nachvollziehbarkeit auf Seiten des Letztverbrauchers. Dies könnte dazu führen, dass sich die Glaubwürdigkeit von Regionalstrom verringert und sich Regionalstrom zu einem reinen Marketinginstrument entwickelt, was den ohnehin geringen positiven Effekt auf die lokale Akzeptanz unterminiert [17, S. 90]. Interessant ist im Zusammenhang mit der lokalen Akzeptanz die Frage, inwieweit ein Zubau von Erneuerbare-Energien-Anlagen durch eine Vergünstigung bei regionalem Strombezug von Letztverbrauchern akzeptiert würde. Dies könnte die wahrgenommene Fairness [vgl. 74] der Bevölkerung erhöhen, welche wiederum positiv auf die lokale Akzeptanz wirkt [77]. Ob eine solche Vergünstigung gesamtwirtschaftlich sinnvoll ist wurde im Rahmen dieser Untersuchung allerdings nicht analysiert.

Die Stromkennzeichnung, welche zum Ziel hat, die Transparenz im Strommarkt zu erhöhen und Letztverbraucher in die Lage zu versetzen, ihre Strombezugsentscheidung entsprechend ihren Präferenzen auf einer besseren Informationsgrundlage zu treffen [91, S. 12], ist für diese zu komplex und daher nicht nachvollziehbar. Das Regionalnachweisregister dürfte diesen Umstand noch verschärfen [16, 24, 38, 39]. Insbesondere dürfte es für die wenigsten Letztverbraucher verständlich sein, weshalb der Regionalanteil in der Stromkennzeichnung auf den EEG-Anteil begrenzt ist. Auf der anderen Seite zeigen die Experteninterviews, dass die Bedeutung der Stromkennzeichnung für den Letztverbraucher von geringer Bedeutung ist. Dies deckt sich mit den Ergebnissen von Hauser et al. [1], wonach über $80 \%$ der über 2.000 befragten Personen die Stromkennzeichnung ihres Stromtarifs nicht kennen und über der Hälfte der Begriff Stromkennzeichnung unbekannt ist.

Regionalstrom in seiner heutigen Form ist ein Nischenprodukt, dessen zukünftige Entwicklung sich nur schwer prognostizieren lässt. Auf der Angebotsseite führen das Regionalnachweisregister und das Ausscheiden von Erzeugungsanlagen aus der EEG-Förderung ab dem Jahr 2021 dazu, dass mehr Anlagen für Regionalstrom zur Verfügung stehen. Auf der Nachfrageseite lassen das laut Experten momentan geringere Kundenbedürfnis nach Regionalstrom sowie die Entwicklung der Ökostromabsatzmengen der letzten Jahre vermuten, dass sich Regionalstrom nicht zu einem massenfähigen Produkt entwickeln wird, solange die aktuelle Regulatorik bestehen bleibt, im Rahmen derer Regionalstrom als Premiumprodukt ${ }^{2}$ mit einem Aufpreis ggü. nicht regionalem Strom vertrieben wird.

\footnotetext{
${ }^{2}$ Als Premiumprodukte werden im Marketing Produktvariationen und neue Produkte verstanden, die speziell entwickelt, verpackt und dargestellt werden mit dem Ziel, eine bestimmte Zielgruppe zu erreichen [vgl. 92, S. 32, 93, S. 39].
} 
Alternative Produktausgestaltungen von Regionalstrom, wie bspw. lokale Energiemärkte oder CommunityKonzepte, könnten im Vergleich zu reinen Stromlieferverträgen ein höheres Marktpotenzial haben. Diese Produkte verknüpfen Regionalität mit weiteren Werteversprechen, wie bspw. Autonomie, Autarkie oder einem Gemeinschaftsgefühl. Weiterhin bieten diese Produkte für Energieversorgungsunternehmen mehr Flexibilität bei der Produktausgestaltung, z.B. in Form von Bündelangeboten ${ }^{3}$.

\footnotetext{
${ }^{3}$ Als Bündel (englisch: Bundle) werden im Marketing Produkte verstanden, die zusammen angeboten werden.
} 


\section{Kritische Reflexion}

Die vorangegangenen Ausführungen haben gezeigt, welche Vor- und Nachteile Energieversorger im Konzept Regionalstrom sehen. Bei der Interpretation der Ergebnisse sollten jedoch nachfolgende Punkte beachtet werden. Die Experten sind sowohl hinsichtlich der Zuordnung zu Versorgerklassen (siehe Kapitel 4.1) als auch hinsichtlich ihres Unternehmensstandorts nicht gleichverteilt. Die Klasse der überregionalen Versorger ist - trotz einer Vielzahl von Anfragen über mehrere Kanäle - klein (siehe Kapitel 4.2). Infolgedessen ist nicht sichergestellt, dass sich die Ansichten dieser Versorgerklasse ausreichend in den Ergebnissen widerspiegeln. Andererseits könnte das geringe Interesse zur Teilnahme an einem Interview ein Indikator dafür sein und zugleich die Aussagen der übrigen Experten bestätigen, dass das Konzept Regionalstrom für diese Versorger schwieriger umzusetzen ist.

Weiterhin sind die Interviewpartner mit elf Experten aus Baden-Württemberg und Bayern auf den süddeutschen Raum konzentriert. Um die Vergleichbarkeit der erhobenen Daten zu gewährleisten wurden die Interviews unter möglichst gleichen Rahmenbedingungen durchgeführt (siehe Kapitel 3.3). Dennoch bleibt anzumerken, dass im Verlauf der Durchführung der Interviews sowohl neues Sachwissen als auch Erfahrungen bei der Durchführung der Interviews selbst erlangt wurde. Dies kann sich in unbewussten Änderungen im Auftreten gegenüber den Experten widergespiegelt haben. Solche Veränderungen im Interviewprozess sind nie gänzlich auszuschließen. Zuletzt geben die Interviews die subjektive Wahrheit der Experten wider, die für die interviewten Personen und einen bestimmten Zeitpunkt gilt [57, S. 561].

Nicht zuletzt liegt der Einschätzung zur zukünftigen Bedeutung des Konzepts Regionalstrom implizit eine Erwartung zu den Präferenzen von Kunden zugrunde, die i.d.R. deutlich von den aktuell beobachtbaren Präferenzen geprägt ist. Die dynamischen Entwicklungen der letzten Jahre - insbesondere bei Themen im Kontext „Umwelt“ (z.B. bei der Wahrnehmung zur Bedeutung von Klimaschutz) - zeigen jedoch, dass sich Präferenzen durchaus schneller als erwartet ändern könnten. 


\section{Anhang A}

\section{Leitfaden für Experteninterview}

\section{Vorgespräch}

Bedanken für Möglichkeit des Interviews

Vorstellung (Jonathan Müller, Nico Lehmann, KIT-IIP)

Ziel der Befragung

Erlaubnis für Tonspuraufnahme erfragen (Vertraulichkeitserklärung)

Anonymität zusichern

$\square$ Ablauf des Interviews erklären

$\square$ Haben Sie noch Fragen bevor es losgeht?

\section{Interview}

1. Könnten Sie kurz zum Einstieg schildern, was Ihre Aufgabe hier in der Organisation ist und wie Ihr beruflicher Hintergrund aussieht?

2. Inwiefern hat sich Ihr Unternehmen mit dem Thema Regionalstrom bereits befasst/auseinandergesetzt?

(Eher) Ja

- Wie haben Sie es eingeordnet/genutzt/bewertet?

- Was waren die ausschlaggebenden Punkte für Ihre

Einordnung/Nutzung/Bewertung?

- Wie erfolgt bei Ihnen die Umsetzung des

Regionalitätskonzepts (regionales Bilanzkreispooling, RNR, ...)?

\section{(Eher) Nein}

- Weshalb hat sich Ihr Unternehmen nicht (mehr) mit dem Thema Regionalstrom befasst?

- Können Sie darauf genauer eingehen?

3. Was halten Sie vom Regionalnachweisregister, welches Anfang 2019 in Deutschland eingeführt wurde?

- Nutzen Sie das Regionalnachweisregister?

- Welche Vorteile hat das Regionalnachweisregister gegenüber den vorherigen Regelungen?

- Ist das Regionalnachweisregister eine sinnvolle Ergänzung?

- Reichten die bisherigen Rahmenbedingungen für Regionalstrom nicht aus? 
4. Inwiefern könnte das Konzept Regionalstrom (mit und ohne Regionalnachweisen) regulatorisch besser umgesetzt werden?

- Einheitlichere Lösung?

- Leichter verständlich (für Verbraucher)?

- Weniger bürokratisch?

- Bessere Förderung von Bürgerenergiegesellschaften, Energie-Communities, ...?

5. Welche Vorteile sehen Sie in Regionalstrom für den Vertrieb und den Stromeinkauf? Vertrieb

- Image/ Emotionale Beziehung (Grün \& Regional)?

- Zusätzliche/größere Gewinnmarge?

- Wie ist die (Mehr-) Zahlungsbereitschaft der Kunden?

- Welche Vorteile hat das RNR für den Vertrieb?

\section{Stromeinkauf}

- Langfristige Geschäftsbeziehungen mit regionalen Erzeugern?

- Kostenersparnisse?

- Welche Vorteile hat das RNR für den Stromeinkauf?

6. Welche Nachteile/Schwierigkeiten sehen Sie von Regionalstrom im Bereich des Vertriebs und Stromeinkaufs?

\section{Vertrieb}

- Kunde möchte keinen Strom aus Region (Stichwort: NIMBY)?

- Produkt zu kompliziert und daher schwer zu kommunizieren?

- (Mehr-) Zahlungsbereitschaft der Kunden nicht ausreichend?

- Welche Nachteile hat das RNR für den Vertrieb?

\section{Stromeinkauf}

- Mehrkosten durch RN, HKN, Bürokratie und sonstige operative Kosten?

- Mehrkosten von Strom? Was sind Hauptkostentreiber?

- Nicht genügend Erzeuger in der Region?

- Welche Nachteile hat das RNR für den Stromeinkauf?

- Kopplung des RN an das physikalische Produkt Strom?

7. Wie sieht die Zukunft von Regionalstrom aus?

- Welchen Einfluss wird das Ausscheiden der ersten EE-Anlagen aus dem Fördersystem EEG ab 2021 haben?

- Besser/nur nutzbar für Bürgerenergiegesellschaften und kommunale Versorgungsunternehmen (z.B. Stadtwerke) oder auch für überregionale Versorger?

- Welche Rolle spielt Regionalstrom bei der Energiewende? Kann mit Regionalstrom die Akzeptanz der Energiewende vor Ort erhöht werden? 
8. Gibt es von Ihrer Seite aus noch Punkte die Sie ansprechen möchten, die wir aber bisher nicht berücksichtigt haben und die Ihrer Meinung nach wichtig sind? 
Nachgespräch

Für Zeit bedanken

Fragebogen mit Faktenfragen überreichen/ansprechen

$\square$ Erbitten von erneuerter Kontaktaufnahme bezüglich Fragebogen und eventuell entstehender Nachfragen

\section{Zusatzfragen}

9. Was verstehen Sie unter Regionalität?

- $<50 \mathrm{~km}$ (Radius)

- Bundesland

- Gemeinde

- ...

10. Welchen Mehrwert bietet Regionalstrom dem Verbraucher?

- Müsste das Attribut „Regionalität“ mit anderen Attributen kombiniert werden, um für den Verbraucher attraktiver zu sein, z.B. IT-technisch?

- IT: App/Vernetzung

- Community: Gemeinschaftsgefühl

- ...

- „Warm-glow of giving“ 


\section{Anhang B}

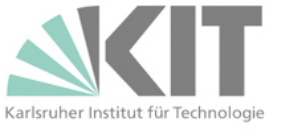

KIT | IIIP | Lehrstuhl für Energiewirtschaft

Herrn/Frau

Name

Unternehmen

Straße

PLZ Ort

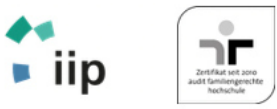

Institut für Industriebetriebslehre und

Industrielle Produktion

Lehrstuhl für Energiewirtschaft

Nico Lehmann

Hertzstr. 16, Geb. 06.33, R110

76187 Karlsruhe

Telefon: 0721-608-44573

Fax: $\quad 0721-608-44682$

E-Mail: nico.lehmann@kit.edu

Web:

Datum: DD.MM.20JJ

Expertengespräch zur Ihren Aktivitäten im Bereich Regionalstrom, insbesondere der Mehrwert für den Vertrieb und die Auswirkungen auf den Einkauf

Sehr geehrter Herr/Frau Mustermann,

im Rahmen einer Dissertation möchte ich Sie um ein Expertengespräch zu Ihren Aktivitäten im Bereich von Regionalstrom bitten.

In der Deutschen Gesellschaft ist das Thema Regionalität sehr aktuell, vor allem im Zusammenhang mit Lebensmitteln ist ein Trend hin zu Produkten aus der Region zu beobachten. Allerdings gewinnt Regionalität auch im Energiesektor zunehmend an Bedeutung und wir legislatorisch von der EU vorangetrieben. So wurde bspw. Anfang 2019 das Regionalnachweisregister beim Umweltbundesamt ins Leben gerufen. Mit diesem können Energieversorger ihren Kundinnen und Kunden regionalen Strom ausweisen. Wir möchten dies nutzen und uns mit dem Thema Regionalstrom genauer beschäftigen, insbesondere mit den Mehrwerten für die verschiedenen Akteure und den Chancen für mehr Akzeptanz in der Gesellschaft bezüglich EE-Anlagen die Ort. Vor allem interessient ertrieb von Energieversorgen einen Mehrwert im Konzept Regionalstrom sieht, welche Auswirkungen dies auf den Einkauf hat und welche Rolle die Einführung des Regionalnachweisregister spielt.

Nach Abschluss der Expertengespräche sollen die gesammelten Informationen helfen, einen Überblick und eine Einschätzung zum Thema Regionalstrom in Deutschland aus Sicht von Energieversorgern zu erhalten, mit besonderem Fokus auf die Vertriebsseite und die Auswirkungen auf den Einkauf. Dies wird in Form einer Bachelorarbeit entstehen, die einer Dissertation zuarbeitet. Um die Sichtweisen der verschiedenen Akteure im Bereich des Regionalstroms adäquat zu berücksichtigen, werden wir in den kommenden Wochen mehrere Interviews mit relevanten Energieversorgungsunternehmen führen. Dies sind sowohl deutschlandweit agierende Versorgungsunternehmen, kommunale Versorger wie bspw. Stadtwerke als auch Bürgerenergiegesellschaften.

Ihre Mitwirkung würde es uns ermöglichen, ein ganzheitliches Bild der aktuellen Situation und der Chancen von Regionalstrom in Deutschland zu bekommen. Das Expertengespräch dauert circa 30 Minuten (zzgl. kurzes Vor- und Nachgespräch). Durchführen würden es Nico Lehmann (im Rahmen seiner Dissertation) und Jonathan Müller (im Rahmen seiner Bachelorarbeit) bei Ihnen vor Ort oder wahlweise an unserem Lehrstuh in Karlsruhe. Die Ergebnisse der Expertengespräche werden ausschließlich in anonymisierter Form veröffentlich, sodass Rückschlüsse auf Einzelpersonen oder Unternehmen nicht möglich sind.

Wir würden uns sehr über eine positive Antwort freuen und schlagen vor, Sie in der kommenden Woche erneut telefonisch zu kontaktieren. Sollten Sie dies nicht wünschen, bitten wir Sie um eine kurze Information per E-Mail.

Besten Dank für Ihre Unterstützung und freundliche Grüße 


\section{Literaturverzeichnis}

[1] E. Hauser et al., „Marktanalyse Ökostrom II: Marktanalyse Ökostrom und HKN, Weiterentwicklung des Herkunftsnachweissystems und der Stromkennzeichnung“, Umweltbundesamt, Climate Change 30/2019, 2019. [Online]. Verfügbar unter: https://www.umweltbundesamt.de/publikationen/marktanalyse-oekostrom-ii. Zugriff am: 22. August 2019.

[2] G. Hoepner, Endkunde. [Online]. Verfügbar unter: https://www.wirtschaftswiki.fh-aachen.de/index.php?title=Endkunde (Zugriff am: 20. August 2020).

[3] PwC, \#energyfacts: Alte Photovoltaik-Anlagen: Ende der Förderung in Sicht. [Online]. Verfügbar unter: https://www.pwc.de/de/energiewirtschaft/pwc-energyfacts-alte-photvoltaik-anlagen-foerderung.pdf (Zugriff am: 31. Juli 2020).

[4] Umweltbundesamt, Hg., „Vierte Fachtagung des Herkunftsnachweisregisters: 26.-27.04.2016“, Dessau-Roßlau, 2016.

[5] W. Lehnert und C. Rühr, „Regionale (Grün-)Stromvermarktung - rechtlich klar geordnet?“, Zeitschrift für Neues Energierecht, Jg. 2019, Nr. 3, S. 190-194, 2019, Art. no. 3.

[6] EDEKA, Genuss aus der Region. [Online]. Verfügbar unter: https://www.edeka.de/nordbayern/unsere-region/regionalitaet/bedeutung-regionalitaet.jsp (Zugriff am: 20. August 2020).

[7] REWE, Gemeinsam für deine Region. [Online]. Verfügbar unter: https://regional.rewe.de/ (Zugriff am: 20. August 2020).

[8] Aldi Süd, Saisonale und regionale Lebensmittel. [Online]. Verfügbar unter: https://nachhaltigkeit.aldi-sued.de/regional-saisonal/ (Zugriff am: 20. August 2020).

[9] Lidl, Sortiment Regionalität. [Online]. Verfügbar unter: https://www.lidl.de/de/sortiment-regionalitaet/s7377410 (Zugriff am: 20. August 2020).

[10] forsa, So will Deutschland essen: Ergebnisse einer repräsentativen Bevölkerungsbefragung. [Online]. Verfügbar unter: https://www.bmel.de/SharedDocs/Downloads/DE/_Ernaehrung/ForsaErn\%C3\%A4hrungsreport2018.pdf?__blob=publicationFile\&v=3 (Zugriff am: 31. Juli 2020).

[11] K. Heinze, S. Xouridas, B. Gebhardt und T. Becker, „Verbraucherpräferenzen gegenüber regionalen Produkten: Ein Vergleich von West- und Ostdeutschland“, Berichte über Landwirtschaft - Zeitschrift für Agrarpolitik und Landwirtschaft, Jg. 92, Nr. 1, 2014, Art. no. 1, doi: 10.12767/BUEL.V92I1.35.

[12] Marketinggesellschaft der niedersächsischen Land- und Ernährungswirtschaft e. V., Definition von Regionalität - Regionalvermarktung Niedersachsen. [Online]. Verfügbar unter: http://www.regionalvermarktung-niedersachsen.de/was-ist-regionalitaet/was-ist-regionalitaet/definition-von-regionalitaet/ (Zugriff am: 30. Oktober 2019).

[13] NABU, Regionale Produktion und Vermarktung: Ziele, Rahmenbedingungen, Forderungen. [Online]. Verfügbar unter: https://www.nabu.de/landwirtschaft/regionalvermarktung.pdf (Zugriff am: 31. Juli 2020).

[14] P. C. Peters, R. Madlener und R. Schemm, „Potenziale zur Erhöhung des regionalen Markenkerns im Stromvertrieb am Beispiel der regionalen Grünstromkennzeichnung gemäß EEG 2017“, Zeitschrift für Energiewirtschaft, Jg. 42, Nr. 1, S. 35-55, 2018, doi: 10.1007/s12398-017-0213-Z.

[15] W. Lehnert, C. Rühr, M. Vollmer und M. Puffe, „Wettbewerbsrecht bei Regionalstromprodukten“. Kurzgutachten, TEXTE 72/2018, 2018. [Online]. Verfügbar unter: https://www.umweltbundesamt.de/sites/default/files/medien/1410/publikationen/2018-09-12_texte_72-2018_wettbewerbsrechtgruenstromkennzeichnung_0.pdf. Zugriff am: 26. Juni 2020.

[16] Umweltbundesamt, ,Workshop 4: Regionale Grünstromkennzeichnung - Ein neues Betätigungsfeld?" in Vierte Fachtagung des Herkunftsnachweisregisters: 26.-27.04.2016, Umweltbundesamt, Hg., Dessau-Roßlau, 2016. [Online]. Verfügbar unter: https://www.umweltbundesamt.de/sites/default/files/medien/372/dokumente/ws4-reggruenstromkennzeichnung.pdf

[17] P. Graichen und F. Zuber, „Regionale Grünstromvermarktung“ in Energiewende und Dezentralität. Zu den Grundlagen einer politisierten Debatte, 2017, S. 83-92. [Online]. Verfügbar unter: https://www.agora-energiewende.de/fileadmin2/Projekte/2016/Dezentralitaet/Agora_Dezentralitaet_WEB.pdf

[18] A. Burkhalter, J. Kaenzig und R. Wüstenhagen, „Kundenpräferenzen für leistungsrelevante Attribute von Stromprodukten“, Zeitschrift für Energiewirtschaft, Jg. 33, Nr. 2, S. 161-172, 2009, doi: 10.1007/s12398-009-0019-8. 
[19] J. Kaenzig, S. L. Heinzle und R. Wüstenhagen, „Whatever the customer wants, the customer gets? Exploring the gap between consumer preferences and default electricity products in Germany", Energy Policy, Jg. 53, S. 311-322, 2013, doi: 10.1016/j.enpol.2012.10.061.

[20] B. J. Kalkbrenner, K. Yonezawa und J. Roosen, „Consumer preferences for electricity tariffs: Does proximity matter?", Energy Policy, Jg. 107, S. 413-424, 2017, doi: 10.1016/j.enpol.2017.04.009.

[21] D. Vecchiato und T. Tempesta, „Public preferences for electricity contracts including renewable energy: A marketing analysis with choice experiments“, Energy, Jg. 88, S. 168-179, 2015, doi: 10.1016/j.energy.2015.04.036.

[22] N. Günther, L. Fait, E. Groh und H. Wetzel, „Gibt es eine Zahlungsbereitschaft für regionalen Grünstrom?“, Energiewirtschaftliche Tagesfragen, Jg. 2019, Nr. 11, S. 35-38, 2019.

[23] N. Lehmann und P. Beikirch, ,Eco-Labeling of green energy tariffs - analysis of the influence of eco-labels on consumer behavior" in 17th International Conference on the European Energy Market (EEM), Stockholm, Sweden, 2020, S. 1-8.

[24] D. Hölder und S. Braig, „Eckpunktepapier zur regionalen Grünstromkennzeichnung - Mehr Transparenz oder teurer Etikettenschwindel? Eine kritische Analyse“, Zeitschrift für Neues Energierecht, Jg. 2016, Nr. 2, S. 117-121, 2016, Art. no. 3.

[25] Bundesnetzagentur, EEG in Zahlen 2018. [Online]. Verfügbar unter: https://www.bundesnetzagentur.de/DE/Sachgebiete/ElektrizitaetundGas/Unternehmen_Institutionen/ErneuerbareEnergien/ZahlenDatenInformationen/zahlenunddaten-node.html (Zugriff am: 20. August 2020).

[26] C. Maaß, J. Güldenberg, J. Mundt, R. Werner und M. Kahles, ,Theoretische Fundierung der regionalen Grünstromkennzeichnung in Deutschland“. Endbericht, Umweltbundesamt, Dessau-Roßlau, Climate Change 17/2017, Juni 2017. [Online]. Verfügbar unter: https://www.umweltbundesamt.de/publikationen/theoretische-fundierung-der-regionalen. Zugriff am: 22. März 2018.

[27] W. Lehnert und F. Sösemann, „Die Vermarktung von EEG-Strom als Grünstrom: Aktueller Rechtsrahmen und geplante Änderungen im EEG 2016“, Zeitschrift für Neues Energierecht, Jg. 2016, Nr. 2, S. 111-116, 2016, Art. no. 2.

[28] Bundesnetzagentur, Hinweis zum Mieterstromzuschlag als eine Sonderform der EEG-Förderung: Version 1.1. [Online]. Verfügbar unter: https://www.bundesnetzagentur.de/SharedDocs/Downloads/DE/Sachgebiete/Energie/Unternehmen_Institutionen/ErneuerbareEnergien/Mieterstrom/Hinweis_Mieterstrom.pdf?_blob=publicationFile\&v=5 (Zugriff am: 31. Juli 2020).

[29] BMWi, Entwurf eines Gesetzes zur Änderung des Erneuerbare-Energien-Gesetzes und weiterer energierechtlicher Vorschriften. Referentenentwurf. [Online]. Verfügbar unter: https://www.bmwi.de/Redaktion/DE/Downloads/Gesetz/referentenentwurf-aenderung-eeg-und-weiterer-energierechtlicher-vorschriften.pdf?__blob=publicationFile\&v=4 (Zugriff am: 6. Oktober 2020).

[30] M. Mulder und S. P. Zomer, „Contribution of green labels in electricity retail markets to fostering renewable energy“, Energy Policy, Jg. 99, S. 100-109, 2016, doi: 10.1016/j.enpol.2016.09.040.

[31] D. Fouquet et al., „Kurzfassung des Abschlussberichts zum Projekt „Unterstützungsleistungen für den effektiven und effizienten Betrieb des Herkunftsnachweisregisters": (Kennzeichen: 03MAP290)“. Stand: 17.09.2014, 17. Sep. 2014. [Online]. Verfügbar unter: https://www.umweltbundesamt.de/sites/default/files/medien/378/dokumente/kurzfassung_unterstuetzungsleistungen_fuer_den_effektiven_und_effizienten_betrieb_des_herkunftsnachweisregisters.pdf. Zugriff am: 6. August 2020.

[32] Umweltbundesamt, Herkunftsnachweisregister (HKNR): Anerkennung ausländischer Herkunftsnachweise. Juristische und energiewirtschaftliche Untersuchung zu Herkunftsnachweisen einzelner Staaten. [Online]. Verfügbar unter: https://www.umweltbundesamt.de/themen/klima-energie/erneuerbare-energien/herkunftsnachweisregister-hknr\#herkunftsnachweise-und-register (Zugriff am: 6. August 2020).

[33] A. Zeiselmair, A. Bogensperger, J. Zarth, H. Michael und F. Haberkorn, „Woher kommt mein Ökostrom wirklich? Mit Blockchain gegen Greenwashing“, Energiewirtschaftliche Tagesfragen, Jg. 2018, Nr. 12, S. 54-56, 2018.

[34] C. Buchmüller, „Plattformökonomie und Blockchain-Technologie - Neue Impulse für die Peer-toPeer-Lieferung von Ökostrom?" Z, Zeitschrift des Instituts für Energie- und Wettbewerbsrecht in der Kommunalen Wirtschaft e.V., Nr. 4, S. 117-125, 2018. [Online]. Verfügbar unter: https://www.ewerk.nomos.de/fileadmin/ewerk/doc/2018/Ewerk_2018_04_01.pdf

[35] BDEW, „Leitfaden "Stromkennzeichnung": Umsetzungshilfe für Elektrizitätsversorgungsunternehmen, Erzeuger und Lieferanten von Strom zu den Bestimmungen über die Stromkennzeichnung (§ 
42 Abs. 1 bis 8 EnWG 2011 i. V. m. $\S \S 78$ und 79 EEG 2017)“. Gültig ab dem Bilanzierungsjahr 2019, Bundesverband der Energie- und Wasserwirtschaft e.V., Berlin, Juli 2020. [Online]. Verfügbar unter: https://www.bdew.de/media/documents/200701_Leitfaden_Stromkennzeichnung_2020.pdf. Zugriff am: 21. August 2020.

[36] C. Herbes, B. Rilling, S. MacDonald, N. Boutin und S. Bigerna, „Are voluntary markets effective in replacing state-led support for the expansion of renewables? - A comparative analysis of voluntary green electricity markets in the UK, Germany, France and Italy“, Energy Policy, Jg. 141, S. 111473, 2020, doi: 10.1016/j.enpol.2020.111473.

[37] V. Hoppenbrock, „Die Vermarktung von Grünstrom nach dem EEG 2014, das Grünstrommarktmodell und die geplante neue regionale Grünstromkennzeichnung“, Zeitschrift für Neues Energierecht, Jg. 2016, Nr. 2, S. 107-110, 2016, Art. no. 1.

[38] L. Conrads, J. Litzenburger und A. K. Meyer, „Die Regionale Grünstromkennzeichnung - systemische Auswirkungen und Nutzbarkeit“, EA.paper \#5, 2016. [Online]. Verfügbar unter: https://www.energieagentur.nrw/content/anlagen/eapaper-5_2016.pdf. Zugriff am: 4. August 2020.

[39] C. Buchmüller, „Regionale Grünstromkennzeichnung - ein neues Geschäftsfeld für Stromversorger?", Zeitschrift des Instituts für Energie- und Wettbewerbsrecht in der Kommunalen Wirtschaft e.V., Jg. 16, Nr. 5, S. 301-306, 2016. [Online]. Verfügbar unter: https://www.ewerk.nomos.de/fileadmin/ewerk/doc/2016/Ewerk_2016_05_00.pdf

[40] Umweltbundesamt, Regionalnachweisregister (RNR). [Online]. Verfügbar unter: https://www.umweltbundesamt.de/themen/klima-energie/erneuerbare-energien/regionalnachweisregister-rnr ( $\mathrm{Zu}$ griff am: 18. August 2020).

[41] „Gesetzentwurf der Fraktionen der CDU/CSU und SPD: Entwurf eines Gesetzes zur Einführung von Ausschreibungen für Strom aus erneuerbaren Energien und zu weiteren Änderungen des Rechts der erneuerbaren Energien (Erneuerbare-Energien-Gesetz - EEG 2016): EEG 2016“ in Drucksache 18/8860, 2016. [Online]. Verfügbar unter: http://dipbt.bundestag.de/dip21/btd/18/088/1808860.pdf

[42] I. Schumacher und P. Würfel, Strategien zur Strombeschaffung in Unternehmen: Energieeinkauf optimieren, Kosten senken. Wiesbaden: Springer Gabler, 2015.

[43] Herkunfts- und Regionalnachweis-Durchführungsverordnung vom 8. November 2018 (BGBl. I S. 1853), Nichtamtliche Lesefassung: HkRNDV, 2018. [Online]. Verfügbar unter: https://www.umweltbundesamt.de/sites/default/files/medien/372/dokumente/hkrndv_und_gebuehrenvo_mit_begruendung_nichtamtliche_lesefassung_20190111.pdf

[44] W. Ouertani, „Umlagesysteme im Energierecht: Eine beihilferechtliche Bewertung unter besonderer Berücksichtigung der EEG-Umlage“, Mohr Siebeck GmbH \& Co. KG, 2018.

[45] K. Fischer und C. Rosenkranz, Handbuch Energiepolitik Österreich. Wien, Berlin, Münster: Lit, 2012.

[46] H. O. Mayer, Interview und Schriftliche Befragung. München: Oldenbourg Wissenschaftsverlag Verlag, 2013.

[47] F. Kleemann, U. Krähnke und I. Matuschek, Interpretative Sozialforschung: Eine Einführung in die Praxis des Interpretierens, 2. Aufl. Springer VS, 2013.

[48] A. Bogner, B. Littig und W. Menz, Interviews mit Experten. Wiesbaden: Springer Fachmedien Wiesbaden, 2014.

[49] A. Bogner, B. Littig und W. Menz, Interviews mit Experten: Eine praxisorientierte Einführung, 1. Aufl. Springer VS, 2014.

[50] S. Matzke, „Methoden der empirischen Sozialforschung“ in Öffentlichkeitsarbeit für Nonprofit-Organisationen, Wiesbaden: Gabler Verlag, 2004, S. 409-426, doi: 10.1007/978-3-322-91231-2_17.

[51] R. Schnell, P. B. Hill und E. Esser, Methoden der empirischen Sozialforschung, 9. Aufl. München: Oldenbourg, 2011.

[52] J. Gläser und G. Laudel, Experteninterviews und qualitative Inhaltsanalyse als Instrumente rekonstruierender Untersuchungen, 4. Aufl. Wiesbaden: VS Verlag, 2010.

[53] A. Bogner, B. Littig und W. Menz, Experteninterviews : Theorien, Methoden, Anwendungsfelder, 3. Aufl. Wiesbaden: Verlag für Sozialwissenschaften, 2009.

[54] RKI, Aktueller Lage-/Situationsbericht des RKI zu COVID-19: Archiv der Situationsberichte des Robert Koch-Instituts zu COVID-19. [Online]. Verfügbar unter: https://www.rki.de/DE/Content/InfAZ/N/Neuartiges_Coronavirus/Situationsberichte/Gesamt.html (Zugriff am: 25. August 2020).

[55] O. Strohm und O. P. Escher, Hg., Unternehmen arbeitspsychologisch bewerten: Ein Mehr-EbenenAnsatz unter besonderer Berücksichtigung von Mensch, Technik und Organisation. Zürich: vdf Hochschulverl. an der ETH Zürich, 1997. 
[56] J. Kirk und M. L. Miller, Reliability and Validity in Qualitative Research. Sage Publications, 1985.

[57] C. Helfferich, „Leitfaden- und Experteninterviews“ in Handbuch Methoden der empirischen Sozialforschung, N. Baur und J. Blasius, Hg., Wiesbaden: Springer Fachmedien Wiesbaden, 2014, S. 559-574, doi: 10.1007/978-3-531-18939-0_39.

[58] C. Helfferich, „Leitfaden- und Experteninterviews“ in Handbuch Methoden der empirischen Sozialforschung, N. Baur und J. Blasius, Hg., Wiesbaden: Springer Fachmedien Wiesbaden, 2019, S. 669-686, doi: 10.1007/978-3-658-21308-4_44.

[59] G. Mey und K. Mruck, Handbuch qualitative Forschung in der Psychologie, 1. Aufl. Springer VS, 2010.

[60] R. Kaiser, Qualitative Experteninterviews: Konzeptionelle Grundlagen und praktische Durchführung. VS Verlag für Sozialwissenschaften, 2014.

[61] R. L. Gordon, Interviewing: Strategies, Techniques and Tactics. Homewood, 1975.

[62] U. Jäger und S. Reinecke, „Expertengespräch“ in Empirische Mastertechniken: Eine anwendungsorientierte Einführung für die Marketing- und Managementforschung, C. Baumgarth, M. Eisend und H. Evanschitzky, Hg., Wiesbaden: Gabler Verlag, 2009, S. 29-76, doi: 10.1007/978-3-83498278-0_2.

[63] P. Mayring, Einführung in die qualitative Sozialforschung: eine Anleitung zu qualitativem Denken, 5. Aufl. Weinheim, Basel: Beltz Verlag, 2002.

[64] f4transkript. Marburg: dr. dresing \& pehl GmbH, 2020. [Online]. Verfügbar unter: https://www.audiotranskription.de/f4

[65] P. Mayring, Qualitative Inhaltsanalyse: Grundlagen und Techniken, 12. Aufl. Weinheim: Beltz, 2015.

[66] f4analyse. Marburg: dr. dresing \& pehl GmbH, 2020. [Online]. Verfügbar unter: https://www.audiotranskription.de/f4-analyse

[67] A. Umathay und A. K. Sinha, „User Experience Strategy for white labeling a software product“ in Indian Conference on Human Computer Interaction, Mumbai, India, 2016, S. 102-110, doi: $10.1145 / 3014362.3014374$.

[68] S. Funke und G. Hempel, „Neue Geschäftsmodelle für Bürgerenergiegemeinschaften nach dem europäischen Clean Energy Package?“", Energiewirtschaftliche Tagesfragen, Jg. 2019, Nr. 3, S. 81-83, 2019.

[69] C. Friege und C. Herbes, „Konzeptionelle Überlegungen zur Vermarktung von Erneuerbaren Energien" in Marketing Erneuerbarer Energien, C. Herbes und C. Friege, Hg., Wiesbaden: Springer Fachmedien Wiesbaden, 2015, S. 3-28.

[70] C. W. Lamb, J. F. Hair und C. McDaniel, Marketing. Cengage Learning, 2012.

[71] P. Kotler und G. M. Armstrong, Principles of marketing, 13. Aufl. Upper Saddle River, N.J, London: Pearson Education, 2010.

[72] K. Bartel, Wettbewerbsprobleme auf dem deutschen Energiemarkt durch Unternehmenszusammenschlüsse: Entflechtung als Mittel der Marktöffnung. LIT Verlag Münster, 2011.

[73] S. Beyer, „Gesamtbewertung von Energieversorgungsunternehmen“ in Branchenorientierte Unternehmensbewertung, J. Drukarczyk und D. Ernst, Hg., 3 Aufl. München: Vahlen, 2010, S. 401-445.

[74] M. Sonnberger und M. Ruddat, „Local and socio-political acceptance of wind farms in Germany“, Technology in Society, Jg. 51, S. 56-65, 2017, doi: 10.1016/j.techsoc.2017.07.005.

[75] U. Liebe und G. M. Dobers, „Decomposing public support for energy policy: What drives acceptance of and intentions to protest against renewable energy expansion in Germany?", Energy Research \& Social Science, Jg. 47, S. 247-260, 2019, doi: 10.1016/j.erss.2018.09.004.

[76] J. Zoellner, P. Schweizer-Ries und C. Wemheuer, „Public acceptance of renewable energies: Results from case studies in Germany“, Energy Policy, Jg. 36, Nr. 11, S. 4136-4141, 2008, doi: 10.1016/j.enpol.2008.06.026.

[77] M. Sonnberger und M. Ruddat, Die gesellschaftliche Wahrnehmung der Energiewende: Ergebnisse einer deutschlandweiten Repräsentativbefragung. [Online]. Verfügbar unter: https://elib.uni-stuttgart.de/bitstream/11682/8911/1/KOMMA-P-Survey-Bericht_300916.pdf (Zugriff am: 26. November 2018).

[78] G. M. Dobers, M. Oehlmann, U. Liebe und J. Meyerhoff, „Einstellungen und Präferenzen zum Ausbau Erneuerbarer Energien“, ÖW, Jg. 30, Nr. 1, S. 16, 2015, doi: 10.14512/OEW300116.

[79] E. Matys, Praxishandbuch Produktmanagement: Grundlagen und Instrumente, 7. Aufl. Frankfurt, New York: Campus Verlag, 2018. 
[80] H. Wildemann, „Produktkannibalisierung erkennen und vermeiden“ in Produkt- und Prozessinnovationen in Wertschöpfungsketten: Tagungsband der Herbsttagung 2007 der Wissenschaftlichen Kommission Produktionswirtschaft im VHB, D. Specht, Hg., Wiesbaden: Gabler, 2008, S. 71-83, doi: 10.1007/978-3-8349-9765-4_4.

[81] Eurostat, Strompreise für Haushalte in den Ländern der EU-28 im Jahr 2019: (in Euro-Cent pro Kilowattstunde). Inklusive aller Steuern; Jahresmittelwerte. [Online]. Verfügbar unter: https://de.statista.com/statistik/daten/studie/197196/umfrage/elektrizitaetspreise-ausgewaehlter-europaeischer-laender/ (Zugriff am: 1. September 2020).

[82] S.-C. Kolm und J. Mercier Ythier, Handbook of the economics of giving, altruism and reciprocity, 1. Aufl. Amsterdam: North-Holland, 2006.

[83] L. A. M. Moutinho und C. S. Chien, Problems in Marketing. London: Sage Publications, 2007.

[84] P. Buxmann und H. Schmidt, Hg., Künstliche Intelligenz: Mit Algorithmen zum wirtschaftlichen Erfolg. Berlin, Heidelberg: Springer Berlin Heidelberg, 2019.

[85] R. Gillenkirch, Besitztumseffekt. [Online]. Verfügbar unter: https://wirtschaftslexikon.gabler.de/definition/besitztumseffekt-53942/version-277004 (Zugriff am: 1. September 2020).

[86] P. Hannen, Immer mehr Abnehmer für Strom aus Post-EEG-Anlagen. [Online]. Verfügbar unter: https://www.pv-magazine.de/2020/09/03/immer-mehr-abnehmer-fuer-strom-aus-post-eeg-anlagen/ (Zugriff am: 4. September 2020).

[87] J. Halberstadt, Globale und nationale First-Mover-Vorteile internetbasierter Geschäftsmodelle. Springer VS, 2014. [Online]. Verfügbar unter: Globale und nationale First-Mover-Vorteile internetbasierter Geschäftsmodelle | Springer

[88] A. Herrmann und F. Huber, Produktmanagement: Grundlagen - Methoden - Beispiele, 2. Aufl. Wiesbaden: Gabler, 2009.

[89] Greenpeace Energy eG, Das Grünstrom-Marktmodell. [Online]. Verfügbar unter: https://www.greenpeace-energy.de/blog/wissen/energiepolitik/oekostrom-markt-modell/ (Zugriff am: 29. Juli 2020).

[90] IZES gGmbH, „Fünfte Fachtagung des Herkunftsnachweisregisters“, Dessau-Roßlau, Dokumentationen 06/2018, Apr. 2018. [Online]. Verfügbar unter: https://www.umweltbundesamt.de/sites/default/files/medien/1410/publikationen/tagungsband_und_vortraege_fuenfte_fachtagung_hknr_0.pdf. Zugriff am: 18. August 2020.

[91] C. Timpe, V. Vürger, D. Seebach und A. Best, „Potenziale und Erfordernisseder Stromkennzeichnung“, Dessau-Roßlau, Climate Change 07/09, Juni 2007. [Online]. Verfügbar unter: https://www.umweltbundesamt.de/sites/default/files/medien/publikation/long/3252.pdf. Zugriff am: 10. September 2020.

[92] V. R. Rao, Handbook of pricing research in marketing. Cheltenham, Glos, U.K, Northampton, Mass: Edward Elgar, 2009. [Online]. Verfügbar unter: http://www.elgaronline.com/view/9781847202406.xml

[93] B. Stewart, Packaging as an effective marketing tool. Leatherhead: Pira International, 1996. 


\title{
Working Paper Series in Production and Energy
}

\author{
recent issues
}

No. 47 Marcus Wiens, Farnaz Mahdavian, Stephen Platt, Frank Schultmann: Optimal Evacuation-Decisions Facing the Trade-Off between EarlyWarning Precision, Evacuation-Cost and Trust - the Warning Compliance Model (WCM)

No. 46 Phuong Khuong, Fabian Scheller, Russell McKenna, Dogan Keles, Wolf Fichtner: Willingness to pay for residential PV: reconciling gaps between acceptance and adoption

No. 45 Christoph Fraunholz, Emil Kraft, Dogan Keles, Wolf Fichtner: The Merge of Two Worlds: Integrating Artificial Neural Networks into Agent-Based Electricity Market Simulation

No. 44 Fritz Braeuer, Rafael Finck, Russell McKenna: Comparing empirical and model-based approaches for calculating dynamic grid emission factors: An application to $\mathrm{CO}_{2}$-minimizing storage dispatch in Germany

No. 43 Russell McKenna, Jann Michael Weinand, Ismir Mulalić, Stefan Petrović, Kai Mainzer, Tobias Preis, Helen Susannah Moat: Quantifying the trade-off between cost-efficiency and public acceptance for onshore wind

No. 42 Thomas Dengiz, Patrick Jochem, Wolf Fichtner: Demand response through decentralized optimization in residential areas with wind and photovoltaics

No. 41 Jann Michael Weinand, Fabian Scheller, Russell McKenna: Reviewing energy system modelling of decentralized energy autonomy

No. 40 Jann Michael Weinand, Sabrina Ried, Max Kleinebrahm, Russell McKenna, Wolf Fichtner: Identification of potential off-grid municipalities with $100 \%$ renewable energy supply

No. 39 Rebekka Volk, Christian Kern, Frank Schultmann: Secondary raw material markets in the C\&D sector: Study on user acceptance in southwest Germany

No. 38 Christoph Fraunholz, Dirk Hladik, Dogan Keles, Dominik Möst, Wolf Fichtner: On the Long-Term Efficiency of Market Splitting in Germany

No. 37 Christoph Fraunholz, Dogan Keles, Wolf Fichtner: On the Role of Electricity Storage in Capacity Remuneration Mechanisms

No. 36 Hansjörg Fromm, Lukas Ewald, Dominik Frankenhauser, Axel Ensslen, Patrick Jochem: A Study on Free-floating Carsharing in Europe Impacts of car2go and DriveNow on modal shift, vehicle ownership, vehicle kilometers traveled, and $\mathrm{CO}_{2}$ emissions in 11 European cities

No. 35 Florian Zimmermann, Andreas Bublitz, Dogan Keles, Wolf Fichtner: Cross-border effects of capacity remuneration mechanisms: the Swiss case

The responsibility for the contents of the working papers rests with the author, not the institute. Since working papers are of preliminary nature, it may be useful to contact the author of a particular working paper about results or caveats before referring to, or quoting, a paper. Any comments on working papers should be sent directly to the author. 


\section{Impressum}

Karlsruher Institut für Technologie

Institut für Industriebetriebslehre und Industrielle Produktion (IIP) Deutsch-Französisches Institut für Umweltforschung (DFIU)

Hertzstr. 16

D-76187 Karlsruhe

KIT - Universität des Landes Baden-Württemberg und

nationales Forschungszentrum in der Helmholtz-Gemeinschaft

Working Paper Series in Production and Energy

No. 48, November 2020

ISSN 2196-7296 